\title{
EXPLICIT CONSTRUCTION OF A CHERN-MOSER CONNECTION FOR CR MANIFOLDS OF CODIMENSION TWO
}

\author{
Gerd Schmalz and Andrea Spiro
}

\begin{abstract}
In the present paper we suggest an explicit construction of a Cartan connection for an elliptic or hyperbolic CR manifold $M$ of dimension six and codimension two, i.e. a pair $(P, \omega)$, consisting of a principal bundle $\pi: P \rightarrow M$ over $M$ and of a Cartan connection form $\omega$ over $P$, satisfying the following property: the (local) CR transformations $f: M \rightarrow M$ are in one to one correspondence with the (local) automorphisms $\hat{f}: P \rightarrow P$ for which $\hat{f}^{*} \omega=\omega$. For any $x \in M$, this construction determines an explicit monomorphism of the stability subalgebra $\operatorname{Lie}\left(\operatorname{Aut}(M)_{x}\right)$ into the Lie algebra $\mathfrak{h}=\operatorname{Lie}(H)$ of the structure group $H$ of $P$.
\end{abstract}

\section{Introduction.}

A $C R$ structure on a smooth manifold $M$ is a pair $(\mathcal{D}, J)$ where $\mathcal{D}$ is a distribution in $T M$ and $J$ is a smooth family of complex structures $J_{x}: \mathcal{D}_{x} \rightarrow \mathcal{D}_{x}$ on the spaces $\mathcal{D}_{x} \subset T_{x} M$. Such geometric structure occurs naturally in studying the geometry of embedded submanifolds of $\mathbb{C}^{n}$. In fact, typical examples of CR structures are given by the pairs $(\mathcal{D}, J)$ on a (sufficiently regular) real submanifold $M \subset \mathbb{C}^{n}$, where $\mathcal{D}$ is the distribution of tangent subspaces $\mathcal{D}_{x}=\left\{v \in T_{x} M: \sqrt{-1} v \in T_{x} M\right\}$ and $J$ is the family of complex structures $J_{x}: \mathcal{D}_{x} \rightarrow \mathcal{D}_{x}$ defined by $J_{x}(v)=\sqrt{-1} v$.

Any CR structure is naturally associated with the pair of distributions $\mathcal{D}^{10}, \mathcal{D}^{01}$ in the complexified tangent space $T^{\mathbb{C}} M$, determined by the subspaces $\mathcal{D}_{x}^{10}, \mathcal{D}_{x}^{01} \subset$ $\mathcal{D}^{\mathbb{C}} \subset T_{x}^{\mathbb{C}} M$ defined by

$$
\mathcal{D}_{x}^{10}=\left\{v \in \mathcal{D}_{x}^{\mathbb{C}}: J v=\sqrt{-1} v\right\}, \mathcal{D}_{x}^{01}=\left\{v \in \mathcal{D}_{x}^{\mathbb{C}}: J v=-\sqrt{-1} v\right\} .
$$

A CR structure $(\mathcal{D}, J)$ is called integrable if the corresponding distribution $\mathcal{D}^{10}$ is involutive, i.e. if for any two complex vector fields $X, Y$ with values in $\mathcal{D}^{10}$ the Lie bracket $[X, Y]$ is still a complex vector field with values in $\mathcal{D}^{10}$. The codimension of a CR structure $(\mathcal{D}, J)$ is the codimension of the distribution $\mathcal{D}$ in $T M$.

A CR structure $(\mathcal{D}, J)$ of codimension one is called Levi non-degenerate if the distribution $\mathcal{D}$ is a contact distribution, i.e. if for any 1 -form $\theta$ with $\operatorname{Ker} \theta_{x}=$ $\mathcal{D}_{x}, x \in M$, one has that $\theta \wedge(d \theta)^{n} \neq 0$. A CR structure $(\mathcal{D}, J)$ of arbitrary codimension is called Levi non-degenerate if $\mathcal{D}$ satisfies a certain set of conditions,

2000 Mathematics Subject Classification. Primary 32V05; Secondary 53C15 53A55.

Key words and phrases. CR manifolds of higher codimension, Invariants for CR structures.

Typeset by $\mathcal{A M}_{\mathcal{M}} \mathcal{S}-\mathrm{T}_{\mathrm{EX}}$ 
which generalizes the condition of Levi non-degeneracy of the CR structures of codimension one. For the exact definition of Levi non-degeneracy in arbitrary codimension, see Definition 2.3 below.

From now on, any CR structure will be assumed to be integrable and Levi nondegenerate.

Now, for a given CR manifold $M$ of codimension $k$, we call osculating quadric at a point $x \in M$ the homogeneous real submanifold of $\mathbb{C} \frac{\operatorname{dim} M+k}{2}$

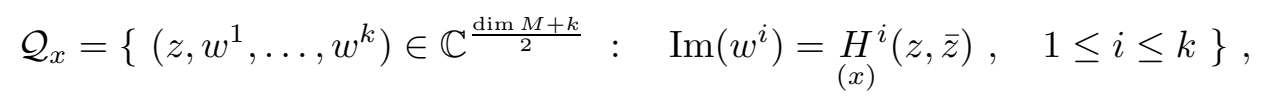

where the $\underset{(x)}{H^{i}}$ 's are the components of the Levi form of the CR structure, evaluated at the point $x$ (for the definition of Levi form of a CR structure, see $\S 2$ below). Geometrically speaking, the osculating quadric at a point $x$ can be characterized as the homogeneous CR manifold, whose CR structure "osculates up to the second order" the CR structure of $M$ at the point $x$. We say that a CR manifold $M$ is of strongly uniform type if all osculating quadrics $\mathcal{Q}_{x}, x \in M$, are equivalent.

For CR structures of codimension one strong uniformity is an automatic consequence of non-degeneracy, due to the classification of quadrics by their signature. In higher codimensions there are only a few cases where non-degenerate quadrics admit a discrete classification. Among them most interesting are certainly those with large automorphism groups. According to [10], this happens only in two situations: either when the real dimension of $\mathcal{Q}$ is 6 and the codimension is 2 , or when the real dimension of $\mathcal{Q}$ is $2 n+n^{2}$ and the codimension is $n^{2}$, where $n$ is the complex dimension of $\mathcal{D}$.

In this paper we discuss the geometry of strongly uniform CR manifolds of dimension six and codimension two.

Strongly uniform CR manifolds of dimension six and codimension two have been intensively studied (see e.g. [14], [8], [9], [10], [7], [11], [12], [16], [18], [19], [6]). In particular, it is known that for such a kind of CR manifolds, there are only three possibilities for the osculating quadric. Using this fact, these manifolds are subdivided into three disjoint classes, namely the elliptic, parabolic and hyperbolic manifolds. Furthermore, in a previous paper J. Slovák and the first author obtained the following theorem, which is consequence of the results in [20] and [5].

Theorem 1.1. [18] Let $M$ be an elliptic or hyperbolic manifold and $\mathcal{Q}$ the osculating quadric at one of its points. Denote also by $G_{\mathcal{Q}}$ the automorphisms group $G_{\mathcal{Q}}=\operatorname{Aut}(\mathcal{Q})$, by $H_{\mathcal{Q}}$ the stability subgroup $H_{\mathcal{Q}}=\operatorname{Aut}(\mathcal{Q})_{0}$ at $0 \in \mathcal{Q}$, and by $\mathfrak{g}_{\mathcal{Q}}$ the Lie algebra $\mathfrak{g}_{\mathcal{Q}}=\operatorname{Lie}\left(G_{\mathcal{Q}}\right)$. Then:

i) there exists a principal bundle $\pi: P(M) \rightarrow M$ with structure group $H_{\mathcal{Q}}$ and a natural injective homomorphism

$$
\imath: \operatorname{Aut}_{l o c}(M) \rightarrow \operatorname{Aut}_{l o c}(P(M))
$$

(called lifting map) from the pseudogroup $\operatorname{Aut}_{l o c}(M)$ of local CR transformations of $M$ into the pseudogroup $\operatorname{Aut}_{l o c}(P(M))$ of local automorphisms 
of bundle $P(M)$, which makes the following diagram commuting

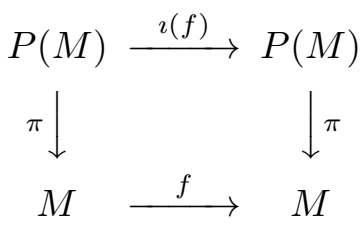

ii) there exists a Cartan connection $\omega_{M}: T P(M) \rightarrow \mathfrak{g}_{\mathcal{Q}}$ such that a diffeomorphism $\tilde{f}: P(M) \rightarrow P(M)$ is equal to $\tilde{f}=\imath(f)$ for some $f \in \operatorname{Aut}(M)$ if and only if $\tilde{f}^{*} \omega_{M}=\omega_{M}$.

Notice that, by Theorem 2.7 in [20], if $\left(P^{\prime}(M), \omega_{M}^{\prime}\right)$ is a pair consisting of a principal bundle $P^{\prime}(M)$, which satisfies (i), and of a Cartan connection $\omega_{M}^{\prime}$, which satisfies (ii) plus a certain set of linear conditions on the curvature, then $\left(P^{\prime}(M), \omega_{M}^{\prime}\right)$ is isomorphic with the pair $\left(P(M), \omega_{M}\right)$ of Theorem 1.1 (i.e. there exists a principal bundle isomorphism $f: P^{\prime}(M) \rightarrow P(M)$ such that $\left.f^{*} \omega_{M}=\omega_{M}^{\prime}\right)$.

From the existence of the invariant Cartan connection $\omega_{M}$, it follows that the action of the stability subgroup $\operatorname{Aut}(M)_{x}, x \in M$, on the fiber $P_{x}=\pi^{-1}(x) \subset$ $P(M)$, commutes with the simply transitive action of $H_{\mathcal{Q}}$ on $P_{x}$. From this it may be inferred that, given a point $u \in P_{x}$, the map

$$
X \in \operatorname{Lie}(\operatorname{Aut}(M))_{x} \mapsto V_{u, X} \in \operatorname{Lie}\left(H_{\mathcal{Q}}\right)
$$

where $V_{u, X} \in \operatorname{Lie}\left(H_{\mathcal{Q}}\right)$ is the element, whose fundamental vector field $V_{u, X}^{*}$ satisfies $\left.V_{u, X}^{*}\right|_{u}=\left.\imath_{*}(X)\right|_{u}$, is a Lie algebra injective homomorphism from $\operatorname{Lie}\left(\operatorname{Aut}(M)_{x}\right)$ into Lie $\left(H_{\mathcal{Q}}\right)$. By this remark, the explicit knowledge of the bundle $P(M)$ and of the lifting map $\imath$ gives an effective tool for reducing several questions on elliptic or hyperbolic manifolds to questions concerning some special subalgebras of $\operatorname{Lie}\left(H_{\mathcal{Q}}\right)$.

It is a disadvantage of the iterative construction, on which the result in [18] is based, that it does not provide an explicit expression of the bundle $P(M)$ and of the Cartan connection (for more details, see also [5], [18] or the review in [1]). On the other hand, the explicit construction of bundle and absolute parallelism in [7] does not give, in general, a Cartan connection and hence, it does not give an explicit expression for the homomorphism between $\operatorname{Lie}\left(\operatorname{Aut}(M)_{x}\right)$ and $\operatorname{Lie}\left(H_{\mathcal{Q}}\right)$.

In this paper we give an explicit and (in our opinion) simple construction of a Cartan connection $\left(P_{\mathrm{CM}}(M), \psi_{C M}\right)$, which satisfies all the claims of Theorem 1.1. However, a direct check shows that, generically, the curvature of $\psi_{C M}$ does not satisfy the linear equations required by Theorem 2.7 in [20]. This means that, generically, the new pair $\left(P_{\mathrm{CM}}(M), \psi_{C M}\right)$ is not isomorphic with the pair $\left(P_{M}, \omega_{M}\right)$. However, we expect that our bundle $P_{\mathrm{CM}}(M)$ admits also another Cartan connection, which does satisfy the conditions of the quoted theorem. If this conjecture is correct, this would imply that the bundle $P_{\mathrm{CM}}(M)$ is equivalent to the bundle $P(M)$.

The bundle $P_{\mathrm{CM}}(M)$ is the exact analogue of the principal bundle associated with a Levi non-degenerate CR manifold of codimension one, introduced by S.S. Chern and J. Moser in [4]. The construction can be roughly described as follows. 
First of all, we consider a principal bundle $\pi_{1}: E(M) \rightarrow M$, with 2-dimensional fibers, formed by all pairs of 1-forms $\left(\theta^{1}, \theta^{2}\right) \in T_{x}^{*} M \times T_{x}^{*} M, x \in M$, such that $\mathcal{D}_{x}=\operatorname{Ker} \theta^{1} \cap \operatorname{Ker} \theta^{2}$ and so that some additional condition, which are specified below, on the corresponding Levi form are satisfied. Secondly, we consider a special class of linear frames $u=\left(e_{1}, \ldots, e_{8}\right)$ at the tangent spaces of $E(M)$, which we call adapted to the $C R$ structure. The bundle $\pi_{2}: P_{\mathrm{CM}}(M) \rightarrow E(M)$ of adapted frames is proved to have a natural structure of an $H_{\mathcal{Q}}$-principal bundle $\pi=\pi_{1} \circ \pi_{2}$ : $P_{\mathrm{CM}}(M) \rightarrow M$ over $M$ and it satisfies claim (i) of Theorem 1.1. In particular, since any CR diffeomorphism $f: M \rightarrow M$ lifts naturally to a map $\hat{f}: E(M) \rightarrow E(M)$ and each map $\hat{f}$ lifts naturally to a diffeomorphism $\hat{\hat{f}}: L(E(M)) \rightarrow L(E(M))$ of the linear frame bundle $L(E(M))$, the lifting map $\imath: \operatorname{Aut}(M) \rightarrow \operatorname{Aut}\left(P_{\mathrm{CM}}(M)\right)$ of Theorem 1.1 (i) is simply the map $\imath: f \mapsto \hat{\hat{f}}$.

Even the construction of the Cartan connection $\psi_{C M}$ is modelled on the arguments used in [4]. In fact, starting from a set of 1-forms $\left\{\omega^{i}, \psi^{j}\right\}$, which represents an arbitrary $\mathfrak{g}_{\mathcal{Q}}$-valued Cartan connection on $P_{\mathrm{CM}}(M)$, we show how to determine a set of real functions $S_{i}^{j}$ on $P(M)$ so that the new collection of 1-forms $\left\{\omega^{i}, \psi^{j}-\sum_{i} S_{i}^{j} \omega^{i}\right\}$ represents a Cartan connection $\psi_{C M}$, which verifies Theorem 1.1 (ii).

We have to recall that, in case $M$ is real analytic and it is presented as a real submanifold of $\mathbb{C}^{4}$, another explicit embedding of $\operatorname{Lie}\left(\operatorname{Aut}(M)_{x}\right)$ into $\operatorname{Lie}\left(H_{\mathcal{Q}}\right)$ can be obtained by studying the normal form of the defining equations for $M$ (see [14], [9], [12]). On the other hand, the approach given here is valid for any smooth manifold $M$ and it is based only on the intrinsic CR geometry of $M$.

Before concluding, we would like to point out that the construction of the ChernMoser bundle can be done also in case $M$ is a parabolic manifold. But, in this case, the bundle does not have a natural structure of a principal bundle over $M$. This is certainly consistent with the results of [20] and [5], which imply neither existence nor non-existence of a canonical Cartan connection on parabolic manifolds. At the best of our knowledge, it is not known if there is any obstruction to the existence of a canonical Cartan connection on parabolic manifolds.

The plan of the paper is the following. In $\S 2$, we discuss a few general facts on CR structures and we give the definition of strongly uniform CR manifolds of dimension six and codimension two. We also give a new proof of the fact that these manifolds are subdivided in exactly three classes. The approach we use is strictly related (even if independent) with the discussion of hyperbolic and elliptic distributions of codimension 2 by A. Čap and M. Eastwood in [3].

In $\S 3$ and $\S 4$, we construct the bundle $P_{\mathrm{CM}}(M)$ of an arbitrary elliptic or hyperbolic manifold $M$. We call it Chern-Moser bundle of $M$. In $\S 5$ we prove that $P_{\mathrm{CM}}(M)$ admits a natural structure of principal bundle over $M$ and we show that its structure group is $G_{\mathcal{Q}}$. In $\S 6$, we construct the Cartan connection $\psi_{C M}$ on $P_{\mathrm{CM}}(M)$ and we illustrate why the pair $\left(P_{\mathrm{CM}}(M), \psi_{C M}\right)$ is generically not isomorphic with the pair $\left(P(M), \omega_{M}\right)$ of Theorem 1.1.

For any real vector space $V$, we denote by $\operatorname{GL}(V, \mathbb{R})$ the group of linear isomorphisms of $V$ into itself. If there is a complex structure $J$ on $V$, we denote by $\mathrm{GL}(V, \mathbb{C}) \subset \mathrm{GL}(V, \mathbb{R})$ the subgroup of isomorphisms commuting with $J$. 


\section{First definitions and preliminaries.}

2.1 Distribution of contact type and Levi non-degenerate CR structures of codimension $k$.

Let $M$ be a manifold of dimension $n$ and let $\mathcal{D} \subset T M$ be a distribution of codimension $k$ on $M$. For any point $x \in M$, we call conormal frame at $x$ any k-tuple $\theta=\left(\theta^{1}, \ldots, \theta^{k}\right)$ of linearly independent 1 -forms $\theta^{i} \in T_{x}^{*} M$ such that $\bigcap_{i=1}^{k} \operatorname{Ker} \theta^{i}=$ $\mathcal{D}_{x}$. It is clear that any conormal frame is a basis for the subspace of 1 -forms vanishing on $\mathcal{D}_{x}$. We call conormal frame bundle of $\mathcal{D}$ the bundle $\pi: E(M, \mathcal{D}) \rightarrow M$ of all conormal frames at the points of $M$ (here, $\pi$ is the natural projection map which sends any conormal frame $\theta \in T_{x}^{*} M \times \cdots \times T_{x}^{*} M$ to the point $\left.x\right)$. Notice that the right action of $\mathrm{GL}_{k}(\mathbb{R})$ on $E(M, \mathcal{D})$ defined by

$\mu: \mathrm{GL}_{k}(\mathbb{R}) \times E(M, \mathcal{D}) \rightarrow E(M, \mathcal{D}), \mu\left(A,\left(\theta^{1}, \ldots, \theta^{k}\right)\right) \stackrel{\text { def }}{=}\left(\left(A^{-1}\right)_{j}^{1} \theta^{j}, \ldots,\left(A^{-1}\right)_{j}^{k} \theta^{j}\right)$

acts transitively on the fibers of $\pi: E(M, \mathcal{D}) \rightarrow M$ and makes $E(M, \mathcal{D})$ a principal bundle over $M$.

With any conormal frame $\theta=\left(\theta^{1}, \ldots, \theta^{k}\right) \in E(M, \mathcal{D})_{x}, x \in M$, we may associate a k-tuple of 2-forms in $\Lambda^{2} \mathcal{D}_{x}$ as follows: consider a smooth section $\tilde{\theta}: \mathcal{U} \subset M \rightarrow$ $E(M, \mathcal{D})$ defined on a neighborhood $\mathcal{U}$ of $x$, such that $\tilde{\theta}_{x}=\theta$; then let us define

$$
\begin{gathered}
\widetilde{d \theta}=\left(\widetilde{d \theta}^{1}, \ldots, \widetilde{d \theta}{ }^{k}\right) \in \Lambda^{2} \mathcal{D}_{x} \times \cdots \times \Lambda^{2} \mathcal{D}_{x}, \\
\widetilde{d \theta}^{i}(X, Y) \stackrel{\text { def }}{=} d\left(\tilde{\theta}^{i}\right)_{x}(X, Y), \quad \text { for any } X, Y \in \mathcal{D}_{x} .
\end{gathered}
$$

It can be checked that the 2-forms $\widetilde{d \theta}^{i} \in \Lambda^{2} \mathcal{D}_{x}$ are independent of the choice of the extension $\tilde{\theta}$ and that they depend uniquely on $\theta=\tilde{\theta}_{x}$. We will call $\widetilde{d \theta}$ the $\mathbb{R}^{k}$-valued 2-form associated with $\theta$.

Definition 2.1. A codimension $k$ distribution $\mathcal{D} \subset T M$ is called of contact type if for any point $x \in M$ and for any conormal frames $\theta=\left(\theta^{1}, \theta^{2}, \ldots, \theta^{k}\right) \in E(M, D)$

a) $\operatorname{Im}(\widetilde{d \theta})=\operatorname{Span}\left\{v \in \mathbb{R}^{k}: v=\widetilde{d \theta}(X, Y)\right.$ for some $\left.X, Y \in \mathcal{D}_{x}\right\}=\mathbb{R}^{k}$;

b) $\operatorname{Ann}(\widetilde{d \theta})=\left\{X \in \mathcal{D}_{x}: \widetilde{d \theta}(X, *)=0\right\}=\{0\}$.

A $C R$ structure of codimension $k$ on a manifold $M$ is a pair $(\mathcal{D}, J)$, where $\mathcal{D}$ is a distribution of codimension $k$ and $J$ is a smooth family of complex structures

$$
J_{x}: \mathcal{D}_{x} \rightarrow \mathcal{D}_{x}
$$

which satisfy the integrability conditions:

$$
\begin{gathered}
J([J X, Y]+[X, J Y]) \in \mathcal{D}, \\
{[J X, J Y]-[X, Y]-J([J X, Y]+[X, J Y])=0 .}
\end{gathered}
$$

We recall that the integrability conditions (2.1) are satisfied if and only if the eigendistributions $\mathcal{D}^{10} \subset T^{\mathbb{C}} M$ and $\mathcal{D}^{01} \subset T^{\mathbb{C}} M$ of $J$, given by the $J$-eigenspaces 
in $\mathcal{D}^{\mathbb{C}}$ corresponding with the eigenvalues $i$ and $-i$, are involutive, i.e. the space of their sections is closed under Lie brackets.

If $(\mathcal{D}, J)$ is a $\mathrm{CR}$ structure of codimension $k$ and $\theta=\left(\theta^{1}, \ldots, \theta^{k}\right)$ is a conormal frame at a point $x \in M$, we call Levi form of $(\mathcal{D}, J)$ associated with $\theta$ the k-tuple of bilinear forms on $\mathcal{D}_{x}$ defined by

$$
L^{\theta}=\left(L^{\theta^{1}}, \ldots, L^{\theta^{k}}\right), \quad L^{\theta^{i}}(X, Y) \stackrel{\text { def }}{=} \widetilde{d \theta^{i}}(X, J Y), \quad \text { for any } X, Y \in \mathcal{D}_{x} .
$$

Using the integrability conditions (2.1), one can check that for any $i$ and for any $X, Y \in \mathcal{D}_{x}$

$$
L^{\theta^{i}}(J X, J Y)=L^{\theta^{i}}(X, Y), \quad L^{\theta^{i}}(X, Y)=L^{\theta^{i}}(Y, X),
$$

and hence $L^{\theta}$ is an $\mathbb{R}^{k}$-valued, $J$-invariant, symmetric, bilinear form on $\mathcal{D}_{x}$.

We also call complex Levi form at $x$ the $\mathbb{C}^{k}$-valued hermitian form defined by

$$
\mathbb{L}^{\theta}(Z, W) \stackrel{\text { def }}{=} \frac{1}{2 i} d \tilde{\theta}_{x}(Z, \bar{W}),
$$

for any $Z, W \in \mathcal{D}_{x}^{\mathbb{C}}$. It is quite simple to check that $\mathbb{L}^{\theta}$ coincides (up to some isomorphism between $\mathbb{C}^{k}$ and $T_{x}^{\mathbb{C}} / D_{x}^{\mathbb{C}}$ ) with the complex Levi form at $x$ as classically defined (see e.g. [2]) and that the Levi form $L^{\theta}$ is (up to a factor) the real part of the complex Levi form.

Definition 2.2. A CR structure $(\mathcal{D}, J)$ of codimension $k$ is called Levi non-degenerate if the underlying distribution $\mathcal{D}$ is of contact type.

Notice that from definitions and (2.4), a CR structure is Levi non-degenerate if and only if any $\mathbb{C}^{k}$-valued Levi form $\mathbb{L}^{\theta}$ satisfies

$$
\begin{gathered}
\operatorname{Im}\left(\mathbb{L}_{x}^{\theta}\right)=\operatorname{Span}\left\{v \in \mathbb{C}^{k}: v=\mathbb{L}_{x}^{\theta}(Z, W) \text { for some } Z, W \in \mathcal{D}_{x}^{\mathbb{C}}\right\}=\mathbb{C}^{k}, \\
\operatorname{Ann}\left(\mathbb{L}_{x}^{\theta}\right) \cap \mathcal{D}_{x}^{10}=\left\{Z \in \mathcal{D}_{x}^{\mathbb{C}}: \mathbb{L}_{x}^{\theta}(Z, *)=0\right\} \cap \mathcal{D}_{x}^{10}=\{0\} .
\end{gathered}
$$

In particular, it is clear that Definition 2.2 is simply a reformulation of the usual Levi non-degeneracy condition on complex valued Levi forms (see e.g. [7]).

\subsection{The three types of CR manifolds of dimension six and codimension two.}

From now, by $(M, \mathcal{D}, J)$ we will always denote an integrable Levi non-degenerate CR manifold of dimension six and codimension two.

Consider such a $\mathrm{CR}$ manifold $(M, \mathcal{D}, J)$ and let $\pi: E(M, \mathcal{D}) \rightarrow M$ be the associated bundle of conormal frames of the distribution $\mathcal{D}$. Recall that $E(M, \mathcal{D})$ is a $\mathrm{GL}_{2}(\mathbb{R})$-principal bundle over $M$. The aim of this subsection is to construct a $\mathrm{GL}_{2}(\mathbb{R})$-equivariant map

$$
\Psi: E(M, \mathcal{D}) \rightarrow \mathbb{P}\left(S_{2 \times 2}(\mathbb{R})\right),
$$

where $\mathbb{P}\left(S_{2 \times 2}(\mathbb{R})\right)$ denotes the projective space of the space of symmetric $2 \times 2$ real matrices and on which we consider the right action of $\mathrm{GL}_{2}(\mathbb{R})$ given by

$$
A \cdot[a]=\left[\left(A^{-1}\right) \cdot a \cdot\left(A^{-1}\right)^{T}\right] \quad \text { for any } A \in \mathrm{GL}_{2}(\mathbb{R}) .
$$

Since the map $\Psi$ will be proved to be $\mathrm{GL}_{2}(\mathbb{R})$-equivariant and any fiber $E_{x}=$ $\pi^{-1}(x) \subset E(M, \mathcal{D})$ is a $G L_{2}(\mathbb{R})$-orbit in $E(M, \mathcal{D})$, we will have a well defined map which assigns to any point $x \in M$ the $\mathrm{GL}_{2}(\mathbb{R})$-orbit in $\mathbb{P}\left(S_{2 \times 2}(\mathbb{R})\right)$ containing the image $\Psi\left(E_{x}\right)$ of $E_{x}=\pi^{-1}(x)$.

In order to define the map $\Psi$, we first need to recall the following lemma. 
Lemma 2.3. Let $(V, J)$ be a 4-dimensional real vector space endowed with a complex structure $J$ and let $\Lambda_{\mathbb{C}}^{2} V^{*}$ be the space of $J$-invariant 2-forms of $V$. Let also $\tau \in \Lambda^{4} V$ be a non-trivial 4-vector on $V$ and $G$ the symmetric bilinear form defined by

$$
\underset{(\tau)}{G}: \Lambda_{\mathbb{C}}^{2} V^{*} \times \Lambda_{\mathbb{C}}^{2} V^{*} \rightarrow \mathbb{R}, \quad \underset{(\tau)}{G}(\alpha, \beta) \stackrel{\text { def }}{=}(\alpha \wedge \beta)(\tau)
$$

Then $G$ is a Lorentz metric on $\Lambda_{\mathbb{C}}^{2} V^{*}$ and the image $\rho\left(\mathrm{GL}_{2}(V, \mathbb{C})\right)$ of the representation

$$
\rho: \mathrm{GL}_{2}(V, \mathbb{C}) \rightarrow \mathrm{GL}\left(\Lambda_{\mathbb{C}}^{2} V^{*}, \mathbb{R}\right), \quad \rho(A) \cdot \alpha=A^{*} \alpha
$$

is equal to the connected component of the identity of the linear conformal group of $\left(\Lambda_{\mathbb{C}}^{2} V, \underset{(\tau)}{G}\right)$.

Proof. Consider a basis $\left(e_{1}, \ldots, e_{4}\right)$ for $V$, which satisfies $J e_{1}=e_{2}, J e_{3}=e_{4}$ and $e_{1} \wedge \cdots \wedge e_{4}=\tau$, and let $\left(e^{1}, \ldots, e^{4}\right)$ be the corresponding dual basis in $V^{*}$. Then we may consider the following basis for $\Lambda_{\mathbb{C}}^{2} V^{*}$

$$
\begin{array}{ll}
\xi^{0}=\frac{1}{2}\left(e^{1} \wedge e^{2}+e^{3} \wedge e^{4}\right), & \xi^{1}=\frac{1}{2}\left(e^{1} \wedge e^{2}-e^{3} \wedge e^{4}\right), \\
\xi^{2}=\frac{1}{2}\left(e^{2} \wedge e^{4}+e^{1} \wedge e^{3}\right), & \xi^{3}=\frac{1}{2}\left(e^{1} \wedge e^{4}-e^{2} \wedge e^{3}\right) .
\end{array}
$$

and observe that the matrix associated with the bilinear form $G$ in this basis is $(\tau)$

$$
\left(\underset{(\tau)}{G}\left(\xi^{i}, \xi^{j}\right)\right)=\left(\begin{array}{cccc}
1 & 0 & 0 & 0 \\
0 & -1 & 0 & 0 \\
0 & 0 & -1 & 0 \\
0 & 0 & 0 & -1
\end{array}\right)
$$

i.e. $\underset{(\tau)}{G}$ is a Lorentz metric. For any complex endomorphism $A \in \operatorname{GL}(V, \mathbb{C})$, let us denote by $A^{*}$ the induced action on $\Lambda_{\mathbb{C}}^{2} V^{*}$. Then, for any $\alpha \in \Lambda_{\mathbb{C}}^{2} V$,

$$
\underset{(\tau)}{G}\left(A^{*} \alpha, A^{*} \alpha\right)=|\operatorname{det}(A)|^{2} \underset{(\tau)}{G}(\alpha, \alpha),
$$

where by $\operatorname{det}(A)$ we mean the determinant of the complex matrix associated with $A$ in the complex basis $\left(e_{1}-i J e_{1}, e_{3}-i J e_{3}\right)$. Then, for any $\alpha \in \Lambda_{\mathbb{C}}^{2} V$, we have that $A^{*} \alpha \in \Lambda_{\mathbb{C}}^{2} V$ and that

$$
\underset{(\tau)}{G}\left(A^{*} \alpha, A^{*} \alpha\right)=|\operatorname{det}(A)|^{2} \underset{(\tau)}{G}(\alpha, \alpha) .
$$

This shows that $(2.9)$ determines a representation of $\mathrm{GL}(V, \mathbb{C})$ as a subgroup of the linear conformal group of $\underset{(\tau)}{G}$. Since the kernel of this representation is $\mathrm{SO}_{2}(\mathbb{R})$, by counting dimension, we get that $\rho(\mathrm{GL}(V, \mathbb{C}))$ is isomorphic to the connected component of the identity of $\mathrm{CO}_{3,1}(\mathbb{R})$.

Now, for any given point $x \in M$, let us fix a non trivial element $\varpi \in \Lambda^{4} \mathcal{D}_{x}$ and let us consider the Lorentz metric $\underset{(\varpi)}{G}$ on $\Lambda^{2} \mathcal{D}_{x}$ defined in (2.8). Notice that if we 
replace $\varpi$ by some other non trivial element of $\Lambda^{4} \mathcal{D}_{x}$, the associated Lorentz metric changes only by multiplication by a non trivial factor.

Let also $E_{x} \subset E(M, \mathcal{D})$ be the fiber over $x$ in $E(M, \mathcal{D})$ and, for any $\theta=\left(\theta^{1}, \theta^{2}\right) \in$ $E_{x}$, denote by $a(\theta)=\left(a^{i j}(\theta)\right)$ the symmetric matrix

$$
a^{i j}(\theta)=\underset{(\varpi)}{G}\left(\widetilde{d \theta}^{i}, \widetilde{d \theta}^{j}\right) .
$$

Using the condition of Levi non-degeneracy, one can check that $a(\theta) \neq 0$ for any $\theta \in E_{x}$ and, by the previous remark, we have that the projective class $[a(\theta)] \in$ $\mathbb{P}\left(S_{2 \times 2}\left(\mathbb{R}^{2}\right)\right)$ is independent of the choice of $\varpi$. In particular, we have a well-defined map

$$
\Psi: E(M, \mathcal{D}) \rightarrow \mathbb{P}\left(S_{2 \times 2}(\mathbb{R})\right), \quad \Psi(\theta)=\left[a^{i j}(\theta)\right] .
$$

If we consider on $\mathbb{P}\left(S_{2 \times 2}(\mathbb{R})\right)$ the right action given in $(2.7)$, it follows from definitions that $\Psi$ is $\mathrm{GL}_{2}(\mathbb{R})$-equivariant and that it is the map we announced at the beginning of this subsection.

Observe that the action $(2.7)$ of $\mathrm{GL}_{2}(\mathbb{R})$ on $\mathbb{P}\left(S_{2 \times 2}(\mathbb{R})\right)$ has exactly three distinct $\mathrm{GL}_{2}(\mathbb{R})$-orbits, namely the orbits of the following three elements

$$
\text { a) }\left[\begin{array}{ll}
1 & 0 \\
0 & 1
\end{array}\right], \quad \text { b) }\left[\begin{array}{ll}
0 & 0 \\
0 & 1
\end{array}\right], \quad \text { c) }\left[\begin{array}{cc}
1 & 0 \\
0 & -1
\end{array}\right] .
$$

This fact leads immediately to the following definition.

Definition 2.4. We say that $x \in M$ is a point of elliptic, parabolic or hyperbolic type if the image $\Psi\left(E_{x}\right)$ of the fiber $E_{x}=\pi^{-1}(x) \subset E(M, \mathcal{D})$ is contained in the $\mathrm{GL}_{2}(\mathbb{R})$-orbit of $\left[\begin{array}{ll}1 & 0 \\ 0 & 1\end{array}\right],\left[\begin{array}{ll}0 & 0 \\ 0 & 1\end{array}\right]$ or $\left[\begin{array}{cc}1 & 0 \\ 0 & -1\end{array}\right]$, respectively.

We say that $M$ has strongly uniform type if all points $x \in M$ have the same type, or, equivalently, if the image $\Psi(E(M, \mathcal{D})) \subset \mathbb{P}\left(S_{2 \times 2}\left(\mathbb{R}^{2}\right)\right)$ is contained in exactly one $\mathrm{GL}_{2}(\mathbb{R})$-orbit of $\mathbb{P}\left(S_{2 \times 2}\left(\mathbb{R}^{2}\right)\right)$. In this case $M$ is called elliptic, parabolic or hyperbolic manifold according to the type of its points.

Now, if $x \in M$ is a point of a $(2 n+k)$-dimensional Levi non-degenerate CR manifold $(M, \mathcal{D}, J)$ of $\mathrm{CR}$ codimension $\mathrm{k}$ and $\theta=\left(\theta^{1}, \ldots, \theta^{k}\right)$ is a conormal frame at the point $x$, we may consider the $\mathbb{C}^{k}$-valued Hermitian form

$$
H_{x}: \mathbb{C}^{n} \times \mathbb{C}^{n} \rightarrow \mathbb{C}^{k}, \quad H_{x}\left(z, z^{\prime}\right) \stackrel{\text { def }}{=} \frac{1}{2 i} \widetilde{d \theta}\left(z, z^{\prime}\right),
$$

where we have identified $\mathbb{C}^{n} \simeq \mathcal{D}_{x}^{10}$. This Hermitian form determines uniquely (up to isomorphisms) the so called osculating quadric of $M$ at the point $x$, namely the quadric

$$
Q_{x} \stackrel{\text { def }}{=}\left\{(z, w) \in \mathbb{C}^{n+k}: \operatorname{Im} w=H_{x}(z, z)\right\} .
$$

Usually (see e.g. [7]), a CR manifold is called strongly uniform if the osculating quadrics $Q_{x}$ and $Q_{x^{\prime}}$ of any two points are equivalent, i.e. if $H_{x^{\prime}}=B \cdot\left(A^{*} H_{x}\right)$ for some $A \in \mathrm{GL}_{n}(\mathbb{C})$ and some $B \in \mathrm{GL}_{k}(\mathbb{R})$. It turns out that if $M$ is of dimension 6 and CR codimension 2, $M$ is of strongly uniform type (according to Definition 2.4) if and only if it is strongly uniform according to this last definition. Actually, we have the following fact, whose proof can be found e.g. in [16]. 
Proposition 2.5. Let $M$ be a Levi non-degenerate, integrable, CR manifold of dimension six and codimension two of strongly uniform type. Then, at any point $x \in M$, the osculating quadric is (up to equivalence) exactly one of the following three quadrics
a) $\quad \operatorname{Im}\left(w_{1}\right)=\operatorname{Im}\left(z_{1} \bar{z}_{2}\right)$
$\operatorname{Im}\left(w_{2}\right)=\operatorname{Re}\left(z_{1} \bar{z}_{2}\right)$
b)
$\operatorname{Im}\left(w_{1}\right)=\left|z_{1}\right|^{2} \quad, \quad \operatorname{Im}\left(w_{2}\right)=\operatorname{Re}\left(z_{1} \bar{z}_{2}\right) \quad ;$
c) $\quad \operatorname{Im}\left(w_{1}\right)=\left|z_{1}\right|^{2} \quad$,
$\operatorname{Im}\left(w_{2}\right)=\left|z_{2}\right|^{2}$

In particular, a) occurs if $M$ is elliptic, b) occurs if $M$ is parabolic and c) occurs if $M$ is hyperbolic.

We want to stress the fact that, for a CR structure $(\mathcal{D}, J)$, the property of being elliptic, parabolic or hyperbolic is mainly a quality of the underlying distribution $\mathcal{D}$ and it is independent of the nature of complex structure $J$. See [3] for some investigations on this fact.

From this point on, we will always assume that $(M, \mathcal{D}, J)$ is a strongly uniform CR manifold of dimension six and codimension two and that it is either elliptic or hyperbolic.

\section{A reduction of the conormal frame bundle $E(M, \mathcal{D})$.}

Consider a strongly uniform $\mathrm{CR}$ manifold $(M, \mathcal{D}, J)$ of elliptic or hyperbolic type and let $\pi: E=E(M, \mathcal{D}) \rightarrow M$ be the conormal frame bundle determined by the distribution $\mathcal{D}$. In all the following, we will denote by $\varpi=\left(\varpi^{1}, \varpi^{2}\right)$ the tautological pair of $E$, that is the pair of 1 -forms $\varpi^{1}$ and $\varpi^{2}$ defined at any point $\theta=\left(\theta^{1}, \theta^{2}\right) \in E_{x} \subset E(M, \mathcal{D})$ by

$$
\left.\varpi^{a}\right|_{\theta}(X, Y)=\theta^{a}\left(\pi_{*}(X), \pi_{*}(Y)\right), a=1,2, \quad \text { for all } X, Y \in T_{\theta} E
$$

Then, we have the following:

Proposition 3.1. Let $\Psi: E \rightarrow \mathbb{P}\left(S_{2 \times 2}(\mathbb{R})\right)$ be the map defined in (2.13) and let $\hat{E}=\Psi^{-1}\left(\left[a_{o}\right]\right)$, where $\left[a_{o}\right]=\left[\begin{array}{ll}1 & 0 \\ 0 & 1\end{array}\right]$ or $\left[\begin{array}{ll}0 & 1 \\ 1 & 0\end{array}\right]$ if $M$ is elliptic or hyperbolic, respectively. Then:

(1) for any point $x \in M$ and any conormal frame $\left(\theta^{1}, \theta^{2}\right) \in \hat{E}_{x}$, there exist four 1-forms $\left(e^{3}, e^{4}, e^{5}, e^{6}\right)$ such that $\left(\theta^{1}, \theta^{2}, e^{3}, \ldots, e^{6}\right)$ is a basis for $T_{x}^{*} M$ such that $\left.e^{4}\right|_{\mathcal{D}_{x}}=\left.J^{*} e^{3}\right|_{\mathcal{D}_{x}},\left.e^{6}\right|_{\mathcal{D}_{x}}=\left.J^{*} e^{5}\right|_{\mathcal{D}_{x}}$ and the 2-forms $\left(\widetilde{d \theta^{1}}, \widetilde{d \theta^{2}}\right)$ can be written in terms of this basis as in the following table: 


\begin{tabular}{|c|c|c|c|}
\hline$M$ & $\widetilde{d \theta^{1}}$ & $\widetilde{d \theta^{2}}$ & $\hat{G}$ \\
\hline Elliptic & $\left(e^{4} \wedge e^{6}+e^{3} \wedge e^{5}\right)_{\mathcal{D}_{x}}$ & $\left(e^{3} \wedge e^{6}-e^{4} \wedge e^{5}\right)_{\mathcal{D}_{x}}$ & $\begin{array}{c}\left\{A \in M_{2,2}(\mathbb{R}): A=\left(\begin{array}{cc}a & b \\
-b & a\end{array}\right) \cdot E\right. \\
\text { where } E=\left(\begin{array}{ll}1 & 0 \\
0 & 1\end{array}\right) \text { or } E=\left(\begin{array}{ll}0 & 1 \\
1 & 0\end{array}\right) \\
\left.\text { and } a^{2}+b^{2} \neq 0\right\} \simeq \mathbb{C}^{*} \times \mathbb{Z}_{2}\end{array}$ \\
\hline Hyperbolic & $\left(e^{3} \wedge e^{4}\right)_{\mathcal{D}_{x}}$ & $\left(e^{5} \wedge e^{6}\right)_{\mathcal{D}_{x}}$ & $\begin{array}{c}\left\{A \in M_{2,2}(\mathbb{R}): A=\left(\begin{array}{ll}a & 0 \\
0 & b\end{array}\right) \cdot E\right. \\
\text { where } E=\left(\begin{array}{cc} \pm 1 & 0 \\
0 & \pm 1\end{array}\right) \text { or } E=\left(\begin{array}{ll}0 & 1 \\
1 & 0\end{array}\right) \\
\left.\text { and } a, b \in \mathbb{R}_{\geq 0}\right\} \simeq \mathbb{R}_{*} \times \mathbb{R}_{*} \times \mathbb{Z}_{2}\end{array}$ \\
\hline
\end{tabular}

Table 1

(2) $\hat{E} \subset E$ is a reduction of $\pi: E \rightarrow M$ with structure group $\hat{G} \subset \mathrm{GL}_{2}(\mathbb{R})$ given in Table 1;

(3) let $\tilde{G} \subset \mathrm{GL}_{2}\left(\mathcal{D}_{x}\right)$ and $\rho: \tilde{G} \rightarrow \hat{G}$ be the subgroups and isomorphisms given in Table 2; then, for any element $\theta \in \hat{E}_{x} \subset E$ and for any $A \in \tilde{G}$, we have that $\widetilde{d \theta} \circ \tilde{A}=\rho(A) \cdot \widetilde{d \theta}$ (in the following table, the elements of $\tilde{G}$ are identified with the matrices which are associated to their action on $\mathcal{D}_{x}^{*}$ w.r.t. a basis $e^{3}, e^{4}, e^{5}$ and $e^{6}$ satisfying (1)):

\begin{tabular}{|c|c|c|}
\hline$M$ & $\tilde{G}$ & $\rho: \tilde{G} \rightarrow \hat{G}$ \\
\hline Elliptic & $\begin{array}{c}\left\{A \in M_{4,4}(\mathbb{C}): A=\left(\begin{array}{ccc}a & -b & 0 \\
b & a & 0 \\
0 & 1 & 0\end{array}\right) \cdot E\right. \\
\text { where } E \text { is } I_{4 \times 4} \text { or }\left(\begin{array}{ccc}1 & 0 & 1 \\
0 & -1 & 0 \\
0 & 0 & 1 \\
0 & 1 & 0\end{array}\right) \\
\left.\text { and } a^{2}+b^{2} \neq 0\right\} \simeq \mathbb{C}^{*} \times \mathbb{Z}_{2}\end{array}$ & $\begin{array}{l}\rho\left(\begin{array}{ccc}a & -b & 0 \\
b & a & 1 \\
0 & 0 & 0\end{array}\right)=\left(\begin{array}{cc}a & b \\
-b & a\end{array}\right) \\
\rho\left(\begin{array}{ccc}1 & 0 & 0 \\
0 & -1 & 0 \\
0 & 0 & 1 \\
0 & 1 & 0\end{array}\right)=\left(\begin{array}{ll}0 & 1 \\
1 & 0\end{array}\right)\end{array}$ \\
\hline Hyperbolic & $\begin{array}{c}\left\{A \in M_{4,4}(\mathbb{R}): A=\left(\begin{array}{ccc}a & 0 & 0 \\
0 & a & b \\
0 & b & 0 \\
0 & b\end{array}\right) \cdot E,\right. \\
\text { where } a, b>0 \text { and } E \text { is } I_{4 \times 4},\left(\begin{array}{ccc}0 & 1 & 0 \\
1 & 0 & 0 \\
0 & 1 & 0\end{array}\right), \\
\left.\left(\begin{array}{ccc}1 & 0 & 0 \\
0 & 1 & 0 \\
0 & 0 & 1 \\
0 & 1\end{array}\right) \text { or }\left(\begin{array}{ccc}0 & 1 & 0 \\
1 & 0 & 1 \\
0 & 1 & 0\end{array}\right)\right\} \simeq \mathbb{R}_{*} \times \mathbb{R}_{*} \times \mathbb{Z}_{2}\end{array}$ & $\begin{array}{c}\rho\left(\begin{array}{ccc}a & 0 & 0 \\
0 & a & 0 \\
0 & b & 0 \\
0 & b\end{array}\right)=\left(\begin{array}{cc}a^{2} & 0 \\
0 & b^{2}\end{array}\right), \rho\left(\begin{array}{ccc}0 & 1 & 0 \\
1 & 0 & 0 \\
0 & 1 & 0 \\
& 0 & 1\end{array}\right)=\left(\begin{array}{cc}-1 & 0 \\
0 & 1\end{array}\right) \\
\rho\left(\begin{array}{ccc}1 & 0 & 0 \\
0 & 1 & 0 \\
0 & 0 & 1 \\
0 & 0\end{array}\right)=\left(\begin{array}{cc}1 & 0 \\
0 & -1\end{array}\right), \rho\left(\begin{array}{ccc}0 & 1 & 0 \\
1 & 0 & 1 \\
0 & 1 & 0\end{array}\right)=\left(\begin{array}{ll}0 & 1 \\
1 & 0\end{array}\right)\end{array}$ \\
\hline
\end{tabular}

Table 2

Proof. (1) Let $\left[a_{o}\right]=\left[\begin{array}{ll}1 & 0 \\ 0 & 1\end{array}\right]$. By definitions, the conormal frames $\theta=\left(\theta^{1}, \theta^{2}\right) \in$ $\Psi^{-1}\left(\left[a_{o}\right]\right) \cap E_{x}$ are exactly those whose corresponding 2 -forms $\left(\widetilde{d \theta}^{1}, \widetilde{d \theta}^{2}\right)$ represent two space-like vectors of an orthonormal basis for the Lorentz metric $G$, for some fixed choice of the 4-vector $\tau$. Choose a basis $\left(\theta^{1}, \theta^{2}, e^{3}, e^{4}, e^{5}, e^{6}\right)$ of $T_{x}^{*} M$, with $\left.e^{4}\right|_{\mathcal{D}_{x}}=\left.J^{*} e^{3}\right|_{\mathcal{D}_{x}}$ and $\left.e^{6}\right|_{\mathcal{D}_{x}}=\left.J^{*} e^{5}\right|_{\mathcal{D}_{x}}$ and with $\left(e^{3} \wedge e^{4} \wedge e^{5} \wedge e^{6}\right)(\tau)=1$, and consider the orthonormal basis $\mathcal{B}=\left(\xi^{0}, \ldots, \xi^{3}\right)$ of $\Lambda_{\mathbb{C}}^{2} \mathcal{D}_{x}$ given by

$$
\xi^{0}=\left.\frac{1}{2}\left(e^{3} \wedge e^{4}+e^{5} \wedge e^{6}\right)\right|_{\mathcal{D}_{x}}, \quad \xi^{1}=\left.\frac{1}{2}\left(e^{3} \wedge e^{4}-e^{5} \wedge e^{6}\right)\right|_{\mathcal{D}_{x}},
$$




$$
\xi^{2}=\left.\frac{1}{2}\left(e^{4} \wedge e^{6}+e^{3} \wedge e^{5}\right)\right|_{\mathcal{D}_{x}}, \quad \xi^{3}=\left.\frac{1}{2}\left(e^{3} \wedge e^{6}-e^{4} \wedge e^{5}\right)\right|_{\mathcal{D}_{x}} .
$$

There always exists an orthonormal basis $\mathcal{B}^{\prime}$ for $\Lambda_{\mathbb{C}}^{2} \mathcal{D}_{x}$, in which the last two vectors are exactly the 2 -forms $\left(\frac{1}{2} \widetilde{d \theta}^{1}, \frac{1}{2} \widetilde{d \theta}^{2}\right)$. We may also assume that $\mathcal{B}^{\prime}$ has the same orientation and the same time-direction of $\mathcal{B}$. Since, by Lemma 2.3, the group $\operatorname{GL}\left(\mathcal{D}_{x}, \mathbb{C}\right)$ acts on $\Lambda_{\mathbb{C}}^{2} \mathcal{D}_{x}$ as the connected component of the identity of $\mathrm{CO}_{3,1}(\mathbb{R})$, there is some element $A \in \mathrm{GL}\left(\mathcal{D}_{x}, \mathbb{C}\right)$ which maps $\mathcal{B}^{\prime}$ into $\mathcal{B}$ and such that $\xi^{2}=$ $\frac{1}{2} A^{*} \widetilde{d \theta}$ and $\xi^{4}=\frac{1}{2} A^{*} \widetilde{d \theta}^{2}$. This implies that, if we consider any $\mathcal{A} \in G L\left(T_{x} M, \mathbb{R}\right)$ which preserves $\mathcal{D}_{x}$ and induces on $\mathcal{D}_{x}$ the transformation $A$, then the 2-forms $\widetilde{d \theta}^{i}$ can be written in terms of $e^{i}=\mathcal{A}^{*} e^{i}$ as in Table $1_{j}$

Similar arguments prove (1) when $\left[a_{o}\right]=\left[\begin{array}{ll}0 & 1 \\ 1 & 0\end{array}\right]$.

(2) From the $\mathrm{GL}_{2}(\mathbb{R})$-equivariance of $\Psi$, it follows immediately that $\hat{E}$ is a reduction of $E$ with structure group equal to the stability subgroup $G_{\left[a_{o}\right]} \subset G L_{2}(\mathbb{R})$ on $\mathbb{P}\left(S_{2 \times 2}(\mathbb{R})\right)$. This stability subgroup is given in Table 1 .

(3) It can be checked using just definitions.

The relevance of the bundle $\hat{E}$ comes from the following fact. For any (local) diffeomorphism $\phi: M \rightarrow M$, let us denote by $\hat{\phi}$ the associated lifted map on

$$
\hat{\phi}: T^{*} M \times T^{*} M \rightarrow T^{*} M \times T^{*} M, \quad \hat{\phi}\left(\theta^{1}, \theta^{2}\right) \stackrel{\text { def }}{=}\left(\left(\phi^{-1}\right)^{*} \theta^{1},\left(\phi^{-1}\right)^{*} \theta^{2}\right) .
$$

Notice that if $\phi: M \rightarrow M$ preserves the distribution $\mathcal{D}$ (i.e. $\phi_{*}(\mathcal{D}) \subset \mathcal{D}$ ) then the lifted map $\hat{\phi}$ is a local diffeomorphism of the conormal frame bundle $E$ into itself such that $\hat{\phi}^{*}(\varpi)=\varpi$. If we consider a (local) CR diffeomorphism $\phi: M \rightarrow M$ (that is such that $\phi_{*}(\mathcal{D}) \subset \mathcal{D}$ and $\phi^{*} J=J$ ), then we have the following crucial property of $\hat{E}$.

Proposition 3.2. Let $\hat{E} \subset E$ be the subbundle defined in Proposition 3.1. Then:

(i) for any (local) $C R$ diffeomorphism $\phi: M \rightarrow M$, the lifted map $\hat{\phi}$ satisfies $\hat{\phi}(\hat{E}) \subset \hat{E}$;

(ii) a (local) diffeomorphism $\varphi: \hat{E} \rightarrow \hat{E}$ is the lifted map $\varphi=\hat{\phi}$ of some (local) diffeomorphism $\phi: M \rightarrow M$ preserving $\hat{E}$ (not necessarily CR), if and only if it satisfies

$$
\varphi^{*}\left(\left.\varpi\right|_{\hat{E}}\right)=\left.\varpi\right|_{\hat{E}}
$$

Proof. Let $\left(\theta^{1}, \theta^{2}\right) \in \hat{E}_{x}$, for some $x \in M$, and let $\left(\theta^{1}, \theta^{2}, e^{3}, \ldots, e^{6}\right)$ be a basis of $T_{x}^{*} M$ as in Proposition 3.1 (1). If $\phi: M \rightarrow M$ is a CR diffeomorphism defined on a neighborhood of $x$, one can check that the 1-forms $\theta^{1 \prime}=\phi^{*}\left(\theta^{1}\right), \theta^{2 \prime}=\phi^{*}\left(\theta^{2}\right)$, $e^{i \prime}=\phi^{*}\left(e^{i}\right), i=3, \ldots, 6$, constitute a basis for $T_{\phi(x)}^{*} M$ and the 2 -forms $\widetilde{d \theta^{1 \prime}}$ and $\widetilde{d \theta^{2 \prime}}$ are written in terms of $e^{i \prime}$ as in Proposition 3.1 (1). From this, it follows that $\Psi\left(\theta^{1 \prime}, \theta^{2 \prime}\right)=\Psi\left(\theta^{1}, \theta^{2}\right)=\left[a_{o}\right]$ and that $\hat{\phi}\left(\theta^{1}, \theta^{2}\right) \in \hat{E}$. The claim that $\hat{\phi}\left(\left.\varpi\right|_{\hat{E}}\right)=\left.\varpi\right|_{\hat{E}}$ follows directly from definitions.

The proof that a (local) diffeomorphism $\varphi: \hat{E} \rightarrow \hat{E}$ is the lifted map of some CR transformation of $M$ if and only if (3.4) holds can be obtained by the same line of arguments of Proposition VI.1.3 in [13]. 
Remark 3.3. Notice that, in addition to $\left(\mathcal{D}, J^{(0)}\right)=(\mathcal{D}, J)$ and $\left(\mathcal{D}, J^{(1)}\right)=(\mathcal{D},-J)$, the manifold $M$ carries two natural (almost) CR structures $\left(\mathcal{D}, J^{(i)}\right), i=2,3$, defined as follows.

At a point $x \in M$, pick any pair $\theta=\left(\theta^{1}, \theta^{2}\right) \in \hat{E}_{x}$ and some corresponding 1-forms $\left(e^{3}, e^{4}, e^{5}, e^{6}\right)$ which satisfy Proposition $3.2(1)$. Then $\mathcal{D}_{x}$ splits into the $J$-invariant subspaces $\mathcal{D}_{x}^{1}=\left.\left.\operatorname{Ker} e^{5}\right|_{\mathcal{D}_{x}} \cap \operatorname{Ker} e^{6}\right|_{\mathcal{D}_{x}}$ and $\mathcal{D}_{x}^{2}=\left.\left.\operatorname{Ker} e^{3}\right|_{\mathcal{D}_{x}} \cap \operatorname{Ker} e^{4}\right|_{\mathcal{D}_{x}}$. This splitting is independent of the choice of $\theta$ and of the $e^{i}$ s (see [5]). The CR structures $\left(\mathcal{D}, J^{(2)}\right)$ and $\left(\mathcal{D}, J^{(3)}\right)$ are defined by

$$
J_{x}^{(2)}=\left.J\right|_{\mathcal{D}_{x}^{1}} \oplus\left(-\left.J\right|_{\mathcal{D}_{x}^{2}}\right), \quad J_{x}^{(3)}=\left(-\left.J\right|_{\mathcal{D}_{x}^{1}}\right) \oplus\left(\left.J\right|_{\mathcal{D}_{x}^{2}}\right), \quad x \in M .
$$

It is not difficult to check that any local transformation $\phi: M \rightarrow M$, which preserves $\mathcal{D}$ and such that $\phi_{*}\left(J^{(i)}\right)=J^{(j)}$ for some $0 \leq i, j \leq 3$, has a lift which maps $\hat{E}$ into $\hat{E}$.

\section{The Chern-Moser bundle of an elliptic or hyperbolic manifold.}

In the following, we will continue adopting the notation of the previous section. In particular, $\varpi=\left(\varpi^{1}, \varpi^{2}\right)$ will be used also to denote the restriction of the tautological pair of $E=E(M, \mathcal{D})$ on the reduction $\hat{E} \subset E$.

Moreover, since $E$ is a principal bundle over $M$, with structure group $\mathrm{GL}_{2}(\mathbb{R})$, we may consider the fundamental vector fields $E_{j}^{i *}$ on $E$, which are determined by the elements $E_{j}^{i}=\left(\delta_{j}^{i}\right) \in \mathfrak{g l}_{2}(\mathbb{R})$. Recall the $E_{j}^{i *}$ are vertical vector fields which span at all points the vertical distribution of $E(M, \mathcal{D})$. Finally, we adopt also the following notation.

Notation 4.1. We will use latin letters $a, b, c, d$ to denote indices which run between 1 and 2; we will use the letters $i, j, k, \ell$ to denote indices which run between 3 and 6 ; we will use greek letters $\alpha, \beta, \gamma, \delta$ to denote indices which run between 7 and 8 .

With capital latin letters $I, J, K, L$, we will denote indices which may run between 1 through 8 .

Consider a frame $\left(e_{1}, \ldots, e_{8}\right) \subset T_{\theta} \hat{E}$ at a point of $\hat{E}$ and denote by $\left(e^{1}, \ldots, e^{8}\right) \subset$

$T_{\theta}^{*} \hat{E}$ the associated dual frame. Then we will use the symbols $e_{1}^{1}, e_{1}^{2}, e_{2}^{1}, e_{2}^{2}$ to denote a quadruple of 1-forms, which depends on the forms $e^{7}, e^{8}$ according to the following rules:

i) if $M$ is elliptic, we set $e_{1}^{1}=e_{2}^{2}=e^{7}$ and $-e_{2}^{1}=e_{1}^{2}=e^{8}$;

ii) if $M$ is hyperbolic, we set $e_{1}^{1}=e^{7}, e_{2}^{2}=e^{8}$ and $e_{2}^{1}=e_{1}^{2}=0$.

Furthermore, we define the following $\mathbb{C}$-valued 1-forms: $E^{0}=e^{1}+i e^{2}, E^{1}=e^{3}+i e^{4}$, $E^{2}=e^{5}+i e^{6}, E_{0}^{0}=e_{1}^{1}+i e_{2}^{2}, \Omega=\varpi^{1}+i \varpi^{2}$.

We have now all ingredients to introduce the concept of "adapted frames" and of "Chern-Moser bundle of an elliptic or hyperbolic manifold".

Definition 4.2. A frame $\left(e_{1}, \ldots, e_{N}\right) \subset T_{\theta} \hat{E}$ is called adapted to the $C R$ structure if the following conditions are satisfied:

1) the vectors $e_{7}$ and $e_{8}$ are equal to the vectors $\tilde{e}_{7}, \tilde{e}_{8}$ defined by

$$
\tilde{e}_{7}=\left\{\begin{array}{cc}
\left.E_{1}^{1 *}\right|_{\theta}+\left.E_{2}^{2 *}\right|_{\theta} & \text { if } M \text { is elliptic, } \\
\left.E_{1}^{1 *}\right|_{\theta} & \text { if } M \text { is hyperbolic },
\end{array}\right.
$$




$$
\tilde{e}_{8}=\left\{\begin{array}{cc}
-\left.E_{2}^{1 *}\right|_{\theta}+\left.E_{1}^{2 *}\right|_{\theta} & \text { if } M \text { is elliptic, } \\
\left.E_{2}^{2 *}\right|_{\theta} & \text { if } M \text { is hyperbolic },
\end{array}\right.
$$

2) the vectors $e_{a}, a=1,2$, are such that $\varpi^{a}\left(e_{b}\right)=\delta_{b}^{a}$;

$3)$ the vectors $e_{i}, i=3,4,5,6$, satisfy $J \hat{\pi}_{*}\left(e_{3}\right)= \pm \hat{\pi}_{*}\left(e_{4}\right), J \hat{\pi}_{*}\left(e_{5}\right)= \pm \hat{\pi}_{*}\left(e_{6}\right)$ and the linear equations

$$
\varpi^{a}\left(e_{i}\right)=0, \quad d \varpi^{a}\left(e_{i}, e_{\alpha}\right)=0
$$

for any $a=1,2$ and $\alpha=7,8$ plus the following conditions (here $\Omega=$

\begin{tabular}{|c|c|}
\hline Elliptic & $\begin{array}{c}d \Omega\left(E_{1}, E_{0}\right)=d \Omega\left(\bar{E}_{1}, \bar{E}_{0}\right)=d \Omega\left(E_{2}, \bar{E}_{0}\right)=d \Omega\left(\bar{E}_{2}, E_{0}\right)=0 \\
d \Omega\left(\bar{E}_{1}, E_{0}\right)=d \Omega\left(E_{2}, E_{0}\right)=0 \\
d \Omega\left(\bar{E}_{1}, E_{2}\right)=1 \quad d \Omega\left(E_{1}, \bar{E}_{2}\right)=0 \\
d \Omega\left(E_{1}, \bar{E}_{1}\right)=d \Omega\left(E_{2}, \bar{E}_{2}\right)=d \Omega\left(E_{1}, E_{2}\right)=d \Omega\left(\bar{E}_{1}, \bar{E}_{2}\right)=0\end{array}$ \\
\hline Hyperbolic & $\left\{\begin{array}{c}d \varpi^{a}\left(e_{i}, e_{a}\right)=0 \text { for any } a=1,2 \\
d \varpi^{1}\left(e_{i}, e_{2}\right)=0 \text { if } i=3,4 \text { and } d \varpi^{2}\left(e_{i}, e_{1}\right)=0 \text { if } i=5,6 \\
d \varpi^{1}\left(e_{i}, e_{j}\right)= \begin{cases}1 & \text { if } i=3, j=4 \\
0 & \text { if }\{i, j\} \neq\{3,4\}\end{cases} \\
d \varpi^{2}\left(e_{i}, e_{j}\right)= \begin{cases}1 & \text { if } i=5, j=6 \\
0 & \text { if }\{i, j\} \neq\{5,6\}\end{cases} \end{array}\right.$ \\
\hline
\end{tabular}
$\varpi^{1}+i \varpi^{2}, E_{0}=\frac{1}{2}\left(e_{1}-i e_{2}\right), E_{1}=\frac{1}{2}\left(e_{3}-i e_{4}\right)$ and $\left.E_{2}=\frac{1}{2}\left(e_{5}-i e_{6}\right)\right)$ :

\section{Table 3}

The conditions given in Definition 4.2 can be totally reformulated into conditions on the dual coframe of an adapted frame. Such conditions are the following.

Lemma 4.3. A frame $\left(e_{1}, \ldots, e_{8}\right)$ of a tangent space $T_{\theta} \hat{E}$ is a adapted to the $C R$ structure if and only if the dual coframe $\left(e^{1}, \ldots, e^{8}\right) \subset T_{\theta}^{*} \hat{E}$ satisfies the following conditions:

i) for $\alpha=7,8$, the 1-forms $e^{\alpha}$ satisfy $e^{\alpha}\left(\tilde{e}_{\beta}\right)=\delta_{\beta}^{\alpha}$, where the $\tilde{e}_{\beta}$, with $\beta=7,8$, are the vectors defined in (4.1);

ii) for $a=1,2, e^{a}=\left.\varpi^{a}\right|_{\theta}$;

iii) for $i=3,4,5,6$, the 1 -forms $e^{i}$ vanish on any of the vectors $\tilde{e}_{\beta}, \beta=7,8$, given in (4.1), and there exists a coframe $\left(\theta^{1}, \ldots, \theta^{6}\right)$ in $T_{x}^{*} M, x=\hat{\pi}(\theta)$, such that $\hat{\pi}^{*}\left(\theta^{i}\right)=e^{i}, i=3, \ldots, 6$, and $\left.J^{*} \theta^{3}\right|_{\mathcal{D}_{x}}= \pm \theta^{4}\left|\mathcal{D}_{x}, J^{*} \theta^{5}\right|_{\mathcal{D}_{x}}=$ $\pm\left.\theta^{6}\right|_{\mathcal{D}_{x}}$

iv) the differentials $d \varpi^{a}, a=1,2$, evaluated at the point $\theta$, are equal to the following expressions for some suitable constants $s, s^{\prime}, t, t^{\prime}, \sigma, \tau$ (here $\Omega=$ $\left.\varpi^{1}+i \varpi^{2}, E^{1}=e^{3}+i e^{4}, E^{2}=e^{5}+i e^{6}, E_{0}^{0}=e^{7}+i e^{8}=e_{1}^{1}+i e_{2}^{2}\right)$ : 


\begin{tabular}{|c||c|}
\hline $\mathcal{D}$ & $d \varpi^{a}$ \\
\hline Elliptic & $d \Omega+E_{0}^{0} \wedge \Omega=\bar{E}^{1} \wedge E^{2}+\sigma E^{1} \wedge \bar{\Omega}+\tau \bar{E}^{2} \wedge \bar{\Omega}$ \\
\hline Hyperbolic & $\begin{array}{l}d \varpi^{1}+e_{a}^{1} \wedge \varpi^{a}=e^{3} \wedge e^{4}+s e^{5} \wedge \varpi^{2}+t e^{6} \wedge \varpi^{2} \\
d \varpi^{2}+e_{a}^{2} \wedge \varpi^{a}=e^{5} \wedge e^{6}+s^{\prime} e^{3} \wedge \varpi^{1}+t^{\prime} e^{4} \wedge \varpi^{1}\end{array}$ \\
\hline
\end{tabular}

Table 4

Proof. Let us check that if a coframe $\left(e^{1}, \ldots, e^{8}\right)$ satisfies i) - iv), then the dual basis $\left(e_{1}, \ldots, e_{N}\right)$ is an adapted frame.

First of all, from i), ii) and iii), it is clear that the vectors $e_{\alpha}$, with $\alpha=7,8$, must coincide with the vectors $\tilde{e}_{\alpha}$ of (4.1). Also (2) of Definition 4.2 is immediately satisfied by the vectors $e_{1}$ and $e_{2}$. Finally, if we consider the remaining vectors $e_{i}$, $i=3,4,5,6$, and we plug them into the tautological 1-forms $\left.\varpi^{a}\right|_{\theta}=e^{a}$ and into $\left.d \varpi^{a}\right|_{\theta}$, we see that all conditions of Definition 4.2 (3) are satisfied.

Conversely, assume that $\left(e^{1}, \ldots, e^{8}\right)$ is a coframe, which is dual to an adapted frame. It is clear that i) and iii) are satisfied. Also ii) is satisfied, since $\left.\varpi^{a}\right|_{\theta}$, $a=1,2$, gives the value 1 if and only if it is evaluated to the vector $e_{a}$. Finally, from (3) of Definition 4.2, it follows that, modulo terms of type $\varpi^{a} \wedge e^{\alpha}, a=1,2$, $\alpha=7,8$, the expressions for $\left.d \varpi^{a}\right|_{\theta}$ have to be as in Table 4 . Then, using the action of the vector fields $E_{j}^{i *}$ on the tautological 1 -forms $\varpi^{a}$, one can compute the values $d \varpi^{a}\left(\left(E_{j}^{i}\right)^{*}, e_{a}\right)$ and check directly that the terms of type $\varpi^{a} \wedge e^{\alpha}, a=1,2, \alpha=7,8$, appearing in the expressions for $\left.d \varpi^{a}\right|_{\theta}$, are those given in Table 4 (at this regard, see also the proof of next Lemma 4.4).

It is fair to ask if there exists at least one adapted frame at any point of $\hat{E}$. The answer is yes as it is proved in the following lemma.

Lemma 4.4. There exists at least one adapted frame at any $\theta_{o}=\left(\theta_{o}^{1}, \theta_{o}^{2}\right) \in \hat{E}$.

Proof. Let $x$ be the point $x=\hat{\pi}\left(\theta_{o}\right) \in M$ and consider a local section $\hat{\theta}: \mathcal{U} \subset M \rightarrow$ $\hat{E}$ such that $\hat{\theta}_{x}=\theta_{o}$. Any element $\theta \in \hat{\pi}^{-1}(\mathcal{U}) \subset E(M, \mathcal{D})$ can be written as

$$
\theta=A^{-1} \cdot \hat{\theta}_{y}
$$

where $y=\pi(\theta) \in \mathcal{U}$ and $A \in \mathrm{GL}_{2}(\mathbb{R})$. Moreover, if we consider the 1 -form $\hat{\varpi}=\hat{\pi}^{*} \hat{\theta}$, we have that the tautological pair $\varpi$ can be written at a point $\theta=A^{-1} \cdot \hat{\theta}_{y}$ as

$$
\left.\varpi\right|_{A^{-1} \cdot \hat{\theta}_{y}}=\left.A^{-1} \cdot \hat{\varpi}\right|_{A^{-1} \cdot \hat{\theta}_{y}} .
$$

Therefore, by the fact that $\theta_{o}=\hat{\theta}_{x}$, we get that $d \varpi$ at $\theta_{o}$ can be written as

$$
\left.d \varpi\right|_{\theta_{o}}=-\left.(d A) \wedge \varpi\right|_{\theta_{o}}+\left.d \hat{\varpi}\right|_{\theta_{o}} .
$$

It is not difficult to realize that $(d A)$ is a $2 \times 2$-matrix of 1 -forms

$$
(d A)=\left(\begin{array}{ll}
e_{1}^{1} & e_{2}^{1} \\
e_{1}^{2} & e_{2}^{2}
\end{array}\right)
$$


with entries $e_{j}^{i}$ which satisfy the linear relations described in i) or ii) in Notation 4.1 .

On the other hand, $\left.d \hat{\varpi}\right|_{\theta_{o}}=\hat{\pi}^{*} d \hat{\theta}_{x}$ and, by Proposition 3.1, we can find 1-forms $\hat{e}^{3}, \ldots, \hat{e}^{6}$ in $T_{x}^{*} M$ so that $d \hat{\theta}_{x}^{a}$ can be written as

$$
d \hat{\theta}_{x}^{a}=H_{i j}^{a} \hat{e}^{i} \wedge \hat{e}^{j}+M_{i b}^{a} \hat{e}^{i} \wedge \theta_{o}^{b}+N_{b c}^{a} \theta_{o}^{b} \wedge \theta_{o}^{c},
$$

where the constants $H_{i j}^{a}$ are so that $\widetilde{d \theta_{o}}=\left.d \hat{\theta}\right|_{\mathcal{D}_{x} \times \mathcal{D}_{x}}$ is in one of the forms listed in Table 1. So, if we set

$$
e^{a}=\hat{\pi}^{*} \theta_{o}^{a}, \quad e^{i}=\hat{\pi}^{*} \hat{e}^{i}
$$

we get that the coframe $\left(e^{1}, \ldots, e^{6}, e_{j}^{i}\right)$ satisfies i), ii) and iii) of Lemma 4.3. Moreover, the differentials $\left.d \varpi^{a}\right|_{\theta_{o}}$ are of the form

$$
\left.d \varpi^{a}\right|_{\theta_{o}}+e_{b}^{a} \wedge \varpi^{b}=H_{i j}^{a} e^{i} \wedge e^{j}+M_{i b}^{a} e^{i} \wedge \varpi^{b}+N_{b c}^{a} \varpi^{b} \wedge \varpi^{c}
$$

for some real numbers $M_{i b}^{a}$ and $N_{i b}^{a}$. Now, replacing the 1-forms $e^{i}, e_{b}^{a}$ with other 1 -forms of the kind $e^{i}+\mathcal{A}_{a}^{i} e^{a}$ and $e_{b}^{a}+\mathcal{B}_{b c}^{a} e^{c}+\mathcal{C}_{b i}^{a} e^{i}$ for some constants $\mathcal{A}_{a}^{i}, \mathcal{B}_{b c}^{a}, \mathcal{C}_{b i}^{a}$, one gets another coframe which still satisfies i), ii) and iii) of Lemma 4.3. Moreover, if the coefficients $\mathcal{A}_{a}^{i}$ and $\mathcal{B}_{j a}^{i}$ are suitably chosen, one can obtain that several of the constants $M_{i b}^{a}$ and $N_{b c}^{a}$, which appear in relation (4.5) for this new coframe, are equal to 0 . Choosing the coefficient, so that a maximal number of the constants $M_{i b}^{a}$ and $N_{b c}^{a}$ vanishes, one obtains the equations of Table 4.

Definition 4.5. Let $M$ be an elliptic or hyperbolic manifold. The extended ChernMoser bundle of $M$ is the set $\hat{P}_{\mathrm{CM}}(M)$ of all adapted frames of $\hat{E}$. The Chern-Moser bundle of $M$ is the subset $P_{\mathrm{CM}}(M) \subset \hat{P}_{\mathrm{CM}}(M)$ given by all adapted frames such that $J \hat{\pi}_{*}\left(e_{3}\right)=+\hat{\pi}_{*}\left(e_{4}\right)$ and $J \hat{\pi}_{*}\left(e_{5}\right)=+\hat{\pi}_{*}\left(e_{6}\right)$.

The set $\hat{P}_{\mathrm{CM}}(M)$ has a natural structure of fiber bundle over $M$ given by the projection

$$
\pi=\hat{\pi} \circ \pi_{o}: P_{\mathrm{CM}}(M) \rightarrow M, \quad \hat{\pi}: \hat{E} \rightarrow M, \pi_{o}: P_{\mathrm{CM}}(M) \rightarrow \hat{E} .
$$

The tautological 1-form of $P_{\mathrm{CM}}(M)$ is the 6 -tuple $\omega=\left(\omega^{1}, \ldots, \omega^{6}\right)$, where the $\omega^{i}$ 's are the 1-forms defined by

$$
\pi_{*}(X)=\sum_{i=1}^{6} \omega^{i}(X) \cdot \hat{\pi}_{*}\left(e_{i}\right) \text { or, equivalently, } \omega^{i}(X)=e^{i}\left(\pi_{o *}(X)\right), i=1, \ldots, 6,
$$

for any $X \in T_{u} \hat{P}_{\mathrm{CM}}(M)$ at a frame $u=\left(e_{1}, \ldots, e_{8}\right) \in \hat{P}_{\mathrm{CM}}(M)$.

\section{The Chern-Moser bundle $P_{\mathrm{CM}}(M)$ is a principal bundle over $M$.}

The aim of this section is the proof of Theorems 5.2 and 5.3 below, which claim that both $\hat{P}_{\mathrm{CM}}(M)$ and $P_{\mathrm{CM}}(M)$ admit a natural structure of principal bundle over $M$. Their proofs require a preliminary result, given in the next Proposition 5.1.

5.1 The natural action of the structure group of $\hat{\pi}: \hat{E} \rightarrow M$ on $\hat{P}_{\mathrm{CM}}(M)$.

The first step for Theorem 5.3 consists in showing that the structure group $\hat{G}$ of $\hat{\pi}: \hat{E} \rightarrow M$ admits a lifted action on $\pi_{o}: \hat{P}_{\mathrm{CM}}(M) \rightarrow \hat{E}$. Namely, 
Proposition 5.1. Let $\hat{G}$ be the structure group of $\hat{\pi}: \hat{E} \rightarrow M$ as described in Proposition 3.1. Then there exists a right action of $\hat{G}$ on $\hat{P}_{\mathrm{CM}}(M)$, which commutes with any diffeomorphism of $\hat{P}_{\mathrm{CM}}(M)$ induced by a $C R$ transformation of $M$.

Proof. In the following, for any element $A \in \hat{G}$, we will denote by $\tilde{A}=\rho^{-1}(A) \in \tilde{G}$ the corresponding element in the group $\tilde{G} \subset \mathrm{GL}_{2}(\mathbb{C})$ given in Table 2. Moreover, for any $A \in \hat{G}$, let $R_{A}: \hat{E} \rightarrow \hat{E}$ be the right action of $A$, i.e.

$$
R_{A}\left(\theta^{1}, \theta^{2}\right)=\left(\left(A^{-1}\right)_{a}^{1} \theta^{a},\left(A^{-1}\right)_{b}^{2} \theta^{b}\right) \stackrel{\text { def }}{=}\left(A^{-1}\right) \cdot \theta
$$

Finally, for any frame $u=\left(e_{1}, \ldots, e_{8}\right) \subset T_{\theta} \hat{E}$, let $\Psi_{A}(u)=\left(e_{1}^{\prime}, \ldots, e_{8}^{\prime}\right)$ be the frame

$$
e_{a}^{\prime}=R_{A *}\left(A_{a}^{b} e_{b}\right), \quad e_{i}^{\prime}=R_{A *}\left(\tilde{A}_{i}^{j} e_{j}\right), \quad e_{\alpha}^{\prime}=R_{A *}\left(e_{\alpha}\right)
$$

(we adopt the convention on indices of Notation 4.1). It is simple to check that, for any two elements $A, A^{\prime} \in \hat{G}$

$$
\Psi_{A} \circ \Psi_{A^{\prime}}=\Psi_{A^{\prime} \cdot A}
$$

and hence that the map $A \mapsto \Psi_{A}$ gives a right action of $\hat{G}$ on the space of linear frames of $\hat{E}$. If we show that this action maps adapted frames into adapted frames, we are done.

Assume that $\left(e_{1}, \ldots, e_{8}\right)$ is an adapted frame and let $\left(e^{1}, \ldots, e^{8}\right)$ be the corresponding dual coframe. Since the fundamental vector fields determined by the structure group $\hat{G}$ are mapped into itself by any diffeomorphism $R_{A}$, with $A \in \hat{G}$, it is clear that the vectors $e_{\alpha}^{\prime}, \alpha=7,8$, satisfy (1) of Definition 4.2 whenever the vectors $e_{\alpha}$ 's do. Notice also that, for any $X \in T_{\theta} \hat{E}$

$$
\begin{gathered}
\left.R_{A}^{*}\left(\varpi^{a}\right)(X)\right|_{\theta}=\varpi_{A^{-1} \cdot \theta}^{a}\left(R_{A} *(X)\right)= \\
=\left(\left(A^{-1}\right)_{b}^{a} \theta^{b}\right)\left(\hat{\pi}_{*} \circ R_{A *}(X)\right)=\left(\left(A^{-1}\right)_{b}^{a} \theta^{b}\right)\left(\hat{\pi}_{*}(X)\right)=\left.\left(\left(A^{-1}\right)_{b}^{a} \varpi^{b}\right)(X)\right|_{\theta} .
\end{gathered}
$$

Therefore, for $a, b, c=1,2$,

$$
\varpi^{a}\left(e_{b}^{\prime}\right)=A_{b}^{c} R_{A}^{*}\left(\varpi^{a}\right)\left(e_{c}\right)=A_{b}^{c}\left(A^{-1}\right)_{d}^{a} \delta_{c}^{d}=\delta_{b}^{a},
$$

and hence also (2) of Definition 4.2 is satisfied. Similarly, using (5.2) and the fact that the vectors $e_{i}$ 's satisfy (4.2), one can check that also the vectors $e_{i}^{\prime}=\tilde{A}_{i}^{j} R_{A *}\left(e_{j}\right)$ satisfy (4.2).

It remains to check if the vectors $e_{i}^{\prime}$ satisfy the conditions of Table 1 . But this can be done, just using the explicit expressions of the matrices $A_{b}^{a} \in \hat{G}$ and $\tilde{A}_{j}^{i} \in \tilde{G}$ and the fact that, by construction, $\left.d \varpi^{a}\right|_{A^{-1} \cdot \theta}\left(e_{j}^{\prime}, e_{b}^{\prime}\right)=\tilde{A}_{j}^{i}\left(A^{-1}\right)_{c}^{a} A_{b}^{d} d \varpi^{c}\left(e_{i}, e_{d}\right)$ for any $a, b=1,2$ and any $i, j=3,4,5,6$.

\subsection{The bundle $\pi: \hat{P}_{\mathrm{CM}}(M) \rightarrow \hat{E}$ is a principal bundle (elliptic case).}

Assume that $M$ is elliptic. Recall that, for any adapted frame $\left(e_{1}, \ldots, e_{8}\right)$ at $\theta \in$ $\hat{E}$, with corresponding dual coframe $\left(e^{1}, \ldots, e^{8}\right)$ the following holds (see Notation 4.1)

$$
d \Omega+E_{0}^{0} \wedge \Omega=\bar{E}^{1} \wedge E^{2}+\sigma E^{1} \wedge \bar{\Omega}+\tau \bar{E}^{2} \wedge \bar{\Omega},
$$


where $\sigma$ and $\tau$ are two suitable complex numbers, depending on the frame $\left(e_{i}\right)$.

Now, consider a new coframe $\left(e^{\prime i}\right)$, which satisfies i), ii) and iii) of Lemma 4.3. Assume also that, if $\left(e^{i}\right)$ satisfies (iii) of Lemma 4.3 with $J^{*} \theta^{3}=\epsilon \theta^{4}$ and $J^{*} \theta^{5}=\epsilon^{\prime} \theta^{6}$, for some $\epsilon, \epsilon^{\prime}= \pm 1$, then the new coframe satisfies (iii) of Lemma 4.3 with the same signs $\epsilon$ and $\epsilon^{\prime}$. Then $\left(e^{i}\right)$ is of the form

$$
\begin{gathered}
e^{a \prime}=e^{a} \quad\left(\Rightarrow \Omega^{\prime}=e^{\prime 1}+i e^{\prime 2}=e^{1}+i e^{2}=\Omega\right) \\
E^{i \prime}=C_{j}^{i} E^{j}+B^{i}\left(e^{1}+i e^{2}\right)+B_{b}^{i}\left(e^{1}-i e^{2}\right)=C_{j}^{i} E^{j}+B^{i} \Omega+B_{b}^{i} \bar{\Omega}, \\
E_{0}^{0 \prime}=E_{0}^{0}+\hat{B}_{j} E^{j}+\tilde{B}_{j} \bar{E}^{j}+\hat{A} \Omega+\tilde{A} \bar{\Omega}
\end{gathered}
$$

where $C_{j}^{i}, B_{a}^{i}, \hat{B}_{j}, \tilde{B}_{j}, \hat{A}$ and $\tilde{A}$ are complex numbers. In order to make $\left(e^{i}\right)$ to satisfy also iv) of Lemma 4.3 , we have to require that the constants $C_{j}^{i}, B_{a}^{i}, \hat{B}_{j}$, $\tilde{B}_{j}, \hat{A}$ and $\tilde{A}$ satisfy some additional conditions. In fact, plugging (5.4) - (5.6) into (5.3), we get that

$$
\begin{gathered}
d \Omega=-E_{0}^{0} \wedge \Omega-\hat{B}_{j} E^{j} \wedge \Omega-\tilde{B}_{j} \bar{E}^{j} \wedge \Omega+\tilde{A} \Omega \wedge \bar{\Omega}+ \\
+\overline{C_{1}^{1}} C_{2}^{2} \bar{E}^{1} \wedge E^{2}+\overline{C_{2}^{1}} C_{2}^{2} \bar{E}^{2} \wedge E^{2}+\overline{C_{1}^{1}} C_{1}^{2} \bar{E}^{1} \wedge E^{1}+\overline{C_{2}^{1}} C_{1}^{2} \bar{E}^{2} \wedge E^{1}+ \\
+\overline{C_{i}^{1}} B^{2} \bar{E}^{i} \wedge \Omega+\overline{C_{i}^{1}} B_{b}^{2} \bar{E}^{i} \wedge \bar{\Omega}-\overline{B^{1}} C_{i}^{2} E^{i} \wedge \bar{\Omega}-\overline{B_{b}^{1}} C_{i}^{2} E^{i} \wedge \Omega+ \\
+\sigma^{\prime} C_{i}^{1} E^{i} \wedge \bar{\Omega}+\sigma^{\prime} B^{1} \Omega \wedge \bar{\Omega}+\tau^{\prime} \overline{C_{i}^{2}} \bar{E}^{i} \wedge \bar{\Omega}+\tau^{\prime} \overline{B_{b}^{2}} \Omega \wedge \bar{\Omega} .
\end{gathered}
$$

By comparison of (5.7) with (5.3), we find the conditions

$$
\begin{gathered}
C_{1}^{1} \overline{C_{2}^{2}}=1, \quad C_{2}^{1}=C_{1}^{2}=0, \quad B^{1}=B_{b}^{2}=0, \\
\tilde{B}_{1}=\overline{C_{1}^{1}} B^{2}, \quad \hat{B}_{2}=-\overline{B_{b}^{1}} C_{2}^{2}, \quad \hat{B}_{1}=\tilde{B}_{2}=\tilde{A}=0 .
\end{gathered}
$$

Moreover, we see that the values $\sigma, \tau$ associated with the frame $\left(e_{i}\right)$ and the values $\sigma^{\prime}, \tau^{\prime}$ associated with the frame $\left(e_{i}\right)$ are related by

$$
\sigma^{\prime}=\left(C_{1}^{1}\right)^{-1} \sigma=\overline{C_{2}^{2}} \sigma, \quad \tau^{\prime}=\left(\overline{C_{2}^{2}}\right)^{-1} \tau=C_{1}^{1} \tau .
$$

From such observations, we obtain that $\left(e^{i}\right)$ is a new adapted frame if and only if it is of the form

$$
\begin{gathered}
E^{0 \prime}\left(\stackrel{\text { def }}{=} e^{\prime 1}+i e^{\prime 2}\right)=E^{0}, \quad E^{1 \prime}=\frac{1}{\bar{C}} E^{1}+\bar{F} \overline{E^{0}}, \quad E^{\prime 2}=C E^{2}+H E^{0}, \\
E_{0}^{0 \prime}=E_{0}^{0}-C F E^{2}-\frac{1}{C} H \bar{E}^{1}+A E^{0} .
\end{gathered}
$$

With similar arguments, it can be checked that there is no adapted coframe $\left(e^{\prime i}\right)$, which satisfies (iii) of Lemma 4.3 with signs $J^{*} \theta^{3}=+\epsilon \theta^{4}$ and $J^{*} \theta^{5}=-\epsilon^{\prime} \theta^{6}$ or $J^{*} \theta^{3}=-\epsilon \theta^{4}$ and $J^{*} \theta^{5}=+\epsilon^{\prime} \theta^{6}$.

Finally, using the same arguments of before, we get that any adapted coframe $\left(e_{i}^{\prime}\right)$, which satisfies iii) of Lemma 4.3 with $J^{*} \theta^{3}=-\epsilon \theta^{4}$ and $J^{*} \theta^{5}=-\epsilon^{\prime} \theta^{6}$, has to be of the form

$$
E^{0 \prime}=E^{0}, \quad E^{1 \prime}=-\frac{1}{\bar{C}} \bar{E}^{2}+\bar{F} \overline{E^{0}}, \quad E^{\prime 2}=C \bar{E}^{1}+H E^{0}
$$




$$
E_{0}^{0 \prime}=E_{0}^{0}-\frac{1}{C} H E^{2}-C F \bar{E}^{1}+A E^{0} .
$$

It follows that $\hat{P}_{\mathrm{CM}}(M)$ is a principal bundle over $\hat{E}$, with structure group $G$, which is isomorphic to the following group of matrices, associated with the transformations of the quadruple $\left(E^{0}, \overline{E^{1}}, E^{2}, E_{0}^{0}\right)$ into the quadruple $\left(E^{\prime 0}, \overline{E^{\prime 1}}, E^{\prime 2}, E_{0}^{\prime 0}\right)$ :

$$
\begin{gathered}
G=G^{(1)} \cdot G^{(2)} G^{(1)}=\left\{\left(\begin{array}{cccc}
1 & 0 & 0 & 0 \\
F & \frac{1}{C} & 0 & 0 \\
H & 0 & C & 0 \\
A & \frac{H}{C} & -C F & 1
\end{array}\right) A, C, F, H \in \mathbb{C},\right\}, \\
G^{(2)}=\left\{I_{4 \times 4},\left(\begin{array}{cccc}
1 & 0 & 0 & 0 \\
0 & 0 & 1 & 0 \\
0 & -1 & 0 & 0 \\
0 & 0 & 0 & 1
\end{array}\right),\left(\begin{array}{cccc}
1 & 0 & 0 & 0 \\
0 & -1 & 0 & 0 \\
0 & 0 & -1 & 0 \\
0 & 0 & 0 & 1
\end{array}\right),\left(\begin{array}{cccc}
1 & 0 & 0 & 0 \\
0 & 0 & -1 & 0 \\
0 & 1 & 0 & 0 \\
0 & 0 & 0 & 1
\end{array}\right)\right\}
\end{gathered}
$$

In particular, $\hat{P}_{\mathrm{CM}}(M)$ is a smooth manifold. Note that $G^{(1)}$ is isomorphic to a subgroup of the isotropy $H_{\mathcal{Q}}$, where $\mathcal{Q}$ is the elliptic quadric.

\subsection{The bundle $\pi: \hat{P}_{\mathrm{CM}}(M) \rightarrow \hat{E}$ is a principal bundle (hyperbolic case).}

Assume now that $M$ is hyperbolic. For any adapted frame $\left(e_{1}, \ldots, e_{8}\right)$ at $\theta \in \hat{E}$, with corresponding dual coframe $\left(e^{1}, \ldots, e^{8}\right)$, from Table 4 and adopting Notation 4.1 , we may write that

$$
\begin{aligned}
& \left.d \varpi^{1}\right|_{\theta}=\frac{i}{2} E^{1} \wedge \bar{E}^{1}+\sigma E^{2} \wedge e^{2}+\bar{\sigma} \bar{E}^{2} \wedge e^{2}-e_{1}^{1} \wedge e^{1}, \\
& \left.d \varpi^{2}\right|_{\theta}=\frac{i}{2} E^{2} \wedge \bar{E}^{2}+\tau E^{1} \wedge e^{1}+\bar{\tau} \bar{E}^{1} \wedge e^{1}-e_{2}^{2} \wedge e^{2}
\end{aligned}
$$

for some $\sigma, \tau \in \mathbb{C}$. Assume also that $\left(e^{i}\right)$ satisfies (iii) of Lemma 4.3 with $J^{*} \theta^{3}=\epsilon \theta^{4}$ and $J^{*} \theta^{5}=\epsilon^{\prime} \theta^{6}$, for some fixed values $\epsilon, \epsilon^{\prime}= \pm 1$. Now, from definitions, a new coframe $\left(e^{i}\right)$, which satisfy (i), (ii) and (iii) of Lemma 4.3, with the same signs $\epsilon$, $\epsilon^{\prime}$ in the equations $J^{*} \theta^{\prime 3}=\epsilon \theta^{\prime 4}$ and $J^{*} \theta^{\prime 5}=\epsilon^{\prime} \theta^{\prime 6}$, can be obtained from $\left(e^{i}\right)$ by means of a linear transformation of the following form:

$$
\begin{gathered}
e^{a \prime}=e^{a}, \quad E^{i \prime}=C_{j}^{i} E^{j}+B_{a}^{i} e^{a}, \\
e_{1}^{1 \prime}=e_{1}^{1}+\hat{B}_{j}^{1} E^{j}+\overline{\hat{B}_{j}^{1}} \bar{E}^{j}+A_{a}^{1} e^{a}, \quad e_{2}^{2 \prime}=e_{2}^{2}+\hat{B}_{j}^{2} E^{j}+\overline{\hat{B}_{j}^{2}} \bar{E}^{j}+A_{a}^{2} e^{a},
\end{gathered}
$$

for some $C_{j}^{i}, B_{a}^{i}, \hat{B}_{j}^{i} \in \mathbb{C}$ and $A_{a}^{i} \in \mathbb{R}$. Assuming that the coframe $\left(e^{i}\right)$ satisfies also (iv) (and hence (5.12) and (5.13)), the following conditions have to be satisfied

$$
\begin{gathered}
\left|C_{1}^{1}\right|^{2}=\left|C_{2}^{2}\right|=1, \quad C_{j}^{a}=0 \text { if } a \neq j, \\
B_{2}^{1}=\hat{B}_{2}^{1}=A_{2}^{1}=0, \quad B_{1}^{2}=\hat{B}_{1}^{2}=A_{1}^{2}=0, \\
\hat{B}_{1}^{1}=\frac{i}{2} \bar{B}_{1}^{1} C_{1}^{1}, \quad \hat{B}_{2}^{2}=\frac{i}{2} \bar{B}_{2}^{2} C_{2}^{2} ;
\end{gathered}
$$

there is no restriction on the coefficients $B_{i}^{i} \in \mathbb{C}$ and $A_{1}^{1} \in \mathbb{R}$. 
On the other hand, a similar line of arguments shows that if a new coframe $\left(e^{i}\right)$ satisfies (i), (ii) and (iii) of Lemma 4.3, with the additional conditions $J^{*} \theta^{\prime 3}=-\epsilon \theta^{\prime 4}$ or $J^{*} \theta^{\prime 5}=-\epsilon^{\prime} \theta^{\prime 6}$, then the condition (iv) of Lemma 4.3 can never be satisfied and hence $\left(e^{i}\right)$ cannot be an adapted coframe.

Since this result is independent of the choice of the element in $\hat{P}_{\mathrm{CM}}(M)$, we conclude that $\hat{P}_{\mathrm{CM}}(M)$ is a principal bundle over $\hat{E}$, with structure group isomorphic to the group of matrices

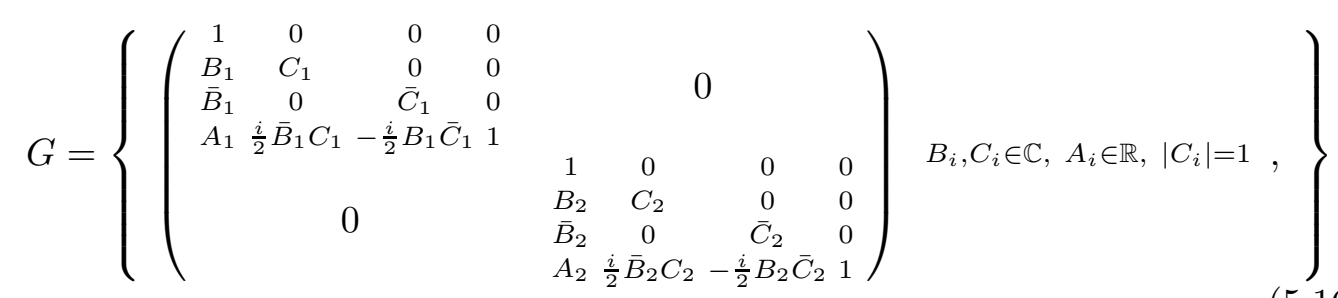

Note that $G$ is isomorphic to a subgroup of the isotropy $H_{\mathcal{Q}}$, where $\mathcal{Q}$ is the hyperbolic quadric.

\subsection{Conclusion.}

The following Theorems are the main result of this section.

Theorem 5.2. Let $M$ be elliptic or hyperbolic, $G$ the group described in (5.11) and (5.16), respectively, and $\hat{G}$ the structure group of $\hat{\pi}: \hat{E} \rightarrow M$. Then the group $\hat{G} \ltimes G$ has a natural right action on $\hat{P}_{\mathrm{CM}}(M)$, determined by the right action of $\hat{G}$ given in Proposition 5.1 and the right action of $G$ described in $\S 5.2$ and $\S 5.3$, and $\pi: \hat{P}_{\mathrm{CM}}(M) \rightarrow M$ is a principal bundle over $M$ with structure group $\hat{G} \ltimes G$.

Moreover, $\hat{G} \ltimes G \simeq H_{\mathcal{Q}} \times \mathbb{Z}_{2}$ if $M$ is elliptic or $\hat{G} \ltimes G \simeq H_{\mathcal{Q}} \times \mathbb{Z}_{2} \times \mathbb{Z}_{2}$ if $M$ is hyperbolic, where $H_{\mathcal{Q}}$ is the stability subgroup of the osculating quadric $\mathcal{Q}$ at the origin.

Proof. The group $H_{\mathcal{Q}}$ is described e. g. in [16]. The existence of the isomorphisms $\hat{G} \ltimes G \simeq H_{\mathcal{Q}} \times \mathbb{Z}_{2}$ or $\hat{G} \ltimes G \simeq H_{\mathcal{Q}} \times \mathbb{Z}_{2} \times \mathbb{Z}_{2}$ follows by comparison of the groups. Finally, the fact that $\hat{G} \ltimes G$ acts transitively on the fibers of $P$ is a direct consequence of the definitions of the actions of $\hat{G}$ and $G$.

Theorem 5.3. The Chern-Moser bundle $P_{\mathrm{CM}}(M)$ of an elliptic or hyperbolic manifold $M$ is a union of connected components of $\hat{P}_{\mathrm{CM}}(M)$. In particular, it is a principal bundle over $M$ with structure group isomorphic to $H_{\mathcal{Q}}$.

Proof. For any point $\theta \in \hat{E}$ and any $u=\left.\left(e_{1}, \ldots, e_{8}\right) \in \hat{P}_{\mathrm{CM}}(M)\right|_{\theta}$, consider the subspace $\tilde{\mathcal{D}}_{\theta, u}=\operatorname{Ker}\left(e^{7}\right) \cap \operatorname{Ker}\left(e^{8}\right)$. The projection $\left.\hat{\pi}_{*}\right|_{T_{\theta} \hat{E}}: T_{\theta} \hat{E} \rightarrow T_{\hat{\pi}(\theta)} M$ induces a linear isomorphism between $\tilde{\mathcal{D}}_{\theta, u}$ and $\mathcal{D}_{\hat{\pi}(\theta)}$. We may consider the induced complex structure $\tilde{J}_{\theta, u}: \tilde{\mathcal{D}}_{\theta, u} \rightarrow \tilde{\mathcal{D}}_{\theta, u}$ defined by

$$
\tilde{J}_{\theta, u}=\left.\left(\left.\hat{\pi}_{*}\right|_{\tilde{\mathcal{D}}_{\theta, u}}\right)^{-1} \circ J \circ \hat{\pi}_{*}\right|_{\tilde{\mathcal{D}}_{\theta, u}} .
$$

So, a frame $u=\left(e_{1}, \ldots, e_{8}\right) \in \hat{P}_{\mathrm{CM}}(M)$ belongs to $P_{\mathrm{CM}}(M)$ if and only if

$$
e^{4}\left(\tilde{J}_{\pi_{o}(u), u}\left(e_{3}\right)\right)=+1, \quad e^{6}\left(\tilde{J}_{\pi_{o}(u), u}\left(e_{5}\right)\right)=+1
$$


Since the functions on the left hand sides are continuous and take values \pm 1 , they are constant on any connected component of $\hat{P}_{\mathrm{CM}}(M)$ and this immediately implies the first claim.

The second claim follows from the fact that, for any $x \in M$, the intersection $\hat{P}_{\mathrm{CM}}(M)_{x} \cap P_{\mathrm{CM}}(M)$ coincides with the orbit of $H_{\mathcal{Q}} \subset \hat{G} \ltimes G$.

\section{A canonical Cartan connection on the Chern-Moser bundle.}

Definition 6.1. Let $P$ be an $H$-principal bundle over $M$ and assume that there exists a Lie algebra $\mathfrak{g}$, which properly contains $\mathfrak{h}=\operatorname{Lie}(H)$ and a representation Ad $: H \rightarrow \operatorname{Aut}(\mathfrak{g})$ which extends the adjoint representation of $\mathfrak{h}$ on $\mathfrak{g}$.

A Cartan connection on $P$ with model $(\mathfrak{g}, \operatorname{Ad}(H))$ is a $\mathfrak{g}$-valued 1 -form $\psi: T P \rightarrow$ $\mathfrak{g}$ such that (here, for any $h \in H, R_{h}$ is the right action of $h$ on $P$ ):

i) for any $u \in P, \psi_{u}: T_{u} P \rightarrow \mathfrak{g}$ is a linear isomorphism;

ii) $\psi\left(A^{*}\right)=A$ for any $A \in \mathfrak{h}=\operatorname{Lie}(H)$ (here $A^{*}$ is the fundamental vector field associated with $A$, i.e. the vector field on $P$, whose flow is equal to the 1-parameter family of diffeomorphisms $\left.R_{\exp (t A)}\right)$

iii) $\psi$ is $H$-invariant, i.e. for any $h \in H$

$$
R_{h}^{*} \psi=\operatorname{Ad}_{h^{-1}} \circ \psi \text {. }
$$

As before, for a given elliptic or hyperbolic manifold $M$, we will always denote by $G_{\mathcal{Q}}$ and $H_{\mathcal{Q}}$ the group of automorphisms of the osculating quadric $\mathcal{Q}$ and the stability subgroup at the origin, respectively. Finally, we set $\mathfrak{g}_{\mathcal{Q}}=\operatorname{Lie}\left(G_{\mathcal{Q}}\right)$ and $\mathfrak{h}_{\mathcal{Q}}=\operatorname{Lie}\left(H_{\mathcal{Q}}\right)$.

By standard facts on elliptic or hyperbolic quadrics (see e.g. [16]), it is known that the Lie algebra $\mathfrak{g}_{\mathcal{Q}}=\operatorname{Lie}\left(G_{\mathcal{Q}}\right)$ is a semisimple Lie algebra isomorphic to $\mathfrak{s u}_{2,1} \oplus \mathfrak{s u}_{2,1}$ (hyperbolic case) or to $\mathfrak{s l}_{3}(\mathbb{C})$ (elliptic case). It also admits a graded decomposition of the form

$$
\mathfrak{g}_{\mathcal{Q}}=\mathfrak{g}_{\mathcal{Q}}^{-2}+\mathfrak{g}_{\mathcal{Q}}^{-1}+\mathfrak{g}_{\mathcal{Q}}^{0}+\mathfrak{g}_{\mathcal{Q}}^{1}+\mathfrak{g}_{\mathcal{Q}}^{2},
$$

with $\left[\mathfrak{g}_{\mathcal{Q}}^{i}, \mathfrak{g}_{\mathcal{Q}}^{j}\right] \subset \mathfrak{g}_{\mathcal{Q}}^{i+j}$ such that $\mathfrak{h}_{\mathcal{Q}}=\mathfrak{g}_{\mathcal{Q}}^{0}+\mathfrak{g}_{\mathcal{Q}}^{1}+\mathfrak{g}_{\mathcal{Q}}^{2}$ and so that the following property holds: if for any $X \in \mathfrak{g}_{\mathcal{Q}}$ we denote by $\hat{X}$ the corresponding infinitesimal transformation on $\mathcal{Q} \subset G_{\mathcal{Q}} / H_{\mathcal{Q}}$, then the map

$$
\imath: \mathfrak{g}_{\mathcal{Q}} \rightarrow T_{0} \mathcal{Q}, \quad X \stackrel{\imath}{\mapsto} \hat{X}_{e H_{\mathcal{Q}}}
$$

induces an isomorphism between $\mathfrak{g}_{\mathcal{Q}}^{-1}$ and the holomorphic tangent space $\mathcal{D}_{0}$ and an isomorphism between $\mathfrak{g}_{\mathcal{Q}}^{-2}+\mathfrak{g}_{\mathcal{Q}}^{-1}$ and $T_{0} \mathcal{Q}$.

Our aim is to construct explicitly a Cartan connection on $P_{\mathrm{CM}}(M)$ with model $\left(\mathfrak{g}_{\mathcal{Q}}, \operatorname{Ad}\left(H_{\mathcal{Q}}\right)\right)$, which is invariant under any $\mathrm{CR}$ transformation of $M$. Any connection which satisfies such property of invariance will be called canonical.

From now on, we will denote by $\left(\epsilon_{i}, V_{A, k}\right)$ the special basis for $\mathfrak{g}_{\mathcal{Q}}$ listed in Appendix. Notice that such a basis is so that:

i) $\left(\epsilon_{1}, \epsilon_{2}\right)$ is a basis for $\mathfrak{g}_{\mathcal{Q}}^{-2},\left(\epsilon_{3}, \ldots, \epsilon_{6}\right)$ is a basis for the subspace $\mathfrak{g}_{\mathcal{Q}}^{-1} \subset \mathfrak{g}_{\mathcal{Q}}$ and

$$
J \imath\left(\epsilon_{3}\right)=\imath\left(\epsilon_{4}\right), \quad J \imath\left(\epsilon_{5}\right)=\imath\left(\epsilon_{6}\right)
$$


(here $\imath$ is the map defined in (6.2) and $J$ is the complex structure of $T_{0} \mathcal{Q}$ );

ii) for any $k=0,1,2$, the elements $\left(V_{A, k}\right)$ form a basis for the subspace $\mathfrak{g}_{\mathcal{Q}}^{k} \subset$ $\mathfrak{g}_{\mathcal{Q}}$

iii) the Lie brackets between the elements $\epsilon_{i}$ are as follows:

\begin{tabular}{|c||c|}
\hline Elliptic & $\begin{array}{c}{\left[\epsilon_{3}, \epsilon_{5}\right]=-\epsilon_{1}, \quad\left[\epsilon_{4}, \epsilon_{6}\right]=-\epsilon_{1}, \quad\left[\epsilon_{3}, \epsilon_{6}\right]=-\epsilon_{2}, \quad\left[\epsilon_{4}, \epsilon_{5}\right]=\epsilon_{2}} \\
{\left[\epsilon_{3}, \epsilon_{4}\right]=\left[\epsilon_{5}, \epsilon_{6}\right]=0}\end{array}$ \\
\hline Hyperbolic & {$\left[\epsilon_{3}, \epsilon_{4}\right]=-\epsilon_{1}, \quad\left[\epsilon_{5}, \epsilon_{6}\right]=-\epsilon_{2}$,} \\
& {$\left[\epsilon_{3}, \epsilon_{5}\right]=\left[\epsilon_{3}, \epsilon_{6}\right]=\left[\epsilon_{4}, \epsilon_{5}\right]=\left[\epsilon_{4}, \epsilon_{6}\right]=0$} \\
\hline
\end{tabular}

Table 5

A given $\mathfrak{g}_{\mathcal{Q}}$-valued 1-form $\psi$ on $P_{\mathrm{CM}}(M)$ can be always written as

$$
\psi=\sum_{i} \epsilon_{i} \psi^{\epsilon_{i}}+\sum V_{A, k} \psi^{V_{A, k}}
$$

where $\psi^{\epsilon_{i}}$ and $\psi^{V_{A, k}}$ denote some suitable $\mathbb{R}$-valued 1-forms on $P_{\mathrm{CM}}(M)$.

Observe that a $\mathfrak{g}_{\mathcal{Q}}$-valued 1-form $\psi$ satisfies (i) of Definition 6.1 if and only if for any $u \in P_{\mathrm{CM}}(M)$ the 1 -forms $\left.\psi^{\epsilon_{i}}\right|_{u}$ and $\left.\psi^{V_{A, k}}\right|_{u}$ are a basis for $T_{u}^{*} P_{\mathrm{CM}}(M)$. If this occurs, we have a natural injective linear homomorphism between $\mathfrak{g}_{\mathcal{Q}}$ and the vectors fields on $P_{\mathrm{CM}}(M)$, namely the correspondence between any element $X \in \mathfrak{g}_{\mathcal{Q}}$ and the unique vector field $\hat{X}$ such that $\psi_{u}(\hat{X})=X$ at any $u \in P_{\mathrm{CM}}(M)$. Such a vector field $\hat{X}$ will be called fundamental vector fields associated with $X \in \mathfrak{g}_{\mathcal{Q}}$ by means of the $\mathfrak{g}_{\mathcal{Q}}$-valued 1-form $\psi$.

Then next Lemma gives a characterization of the Cartan connections amongst the $\mathfrak{g}_{\mathcal{Q}}$-valued 1-forms which satisfy Definition 6.1 (i).

Lemma 6.2. Let $\left(\psi^{\epsilon_{i}}, \psi^{V_{A, k}}\right)$ be a set of $\mathbb{R}$-valued 1-forms on $P_{\mathrm{CM}}(M)$, which are linearly independent at all points of $P_{\mathrm{CM}}(M)$, and let $\psi=\sum_{i} \epsilon_{i} \psi^{\epsilon_{i}}+\sum V_{A, k} \psi^{V_{A, k}}$. For any $X \in \mathfrak{g}_{\mathcal{Q}}$, denote also by $\hat{X}$ the associated fundamental vector field, by means of $\psi$. Then $\psi$ is a Cartan connection modelled on $\left(\mathfrak{g}_{\mathcal{Q}}, \operatorname{Ad}\left(H_{\mathcal{Q}}\right)\right)$ if and only if

(1) the vector fields $\hat{V}_{A, k}$ coincide with the fundamental vector fields $V_{A, k}^{*}$ associated with the elements $V_{A, k} \in \mathfrak{h}_{\mathcal{Q}} \subset \mathfrak{g}_{\mathcal{Q}}$, by means of the right action of $H_{\mathcal{Q}}$ on $P_{\mathrm{CM}}(M)$

(2) for any vector field $\hat{\epsilon}_{i}$ and any element $V_{B, k} \in \mathfrak{h}_{\mathcal{Q}}$

$$
\left[V_{B, k}, \epsilon_{i}\right]=-d \psi\left(V_{B, k}^{*}, \hat{\epsilon}_{i}\right)=\psi\left(\left[V_{B, k}^{*}, \hat{\epsilon}_{i}\right]\right)
$$

(3) $\psi$ is invariant under the element $g_{\mathrm{sym}} \in H_{\mathcal{Q}} \subset \hat{G} \ltimes G$ defined as follows: if $M$ is elliptic, then $g_{\mathrm{sym}}=g_{1} \cdot g_{2}$, where $g_{1}=\left(\begin{array}{cc}1 & 0 \\ 0 & -1\end{array}\right) \in \hat{G}$ and $g_{2} \in G$ is the element which exchanges $\bar{E}^{1}$ with $E^{2}$; if $M$ is hyperbolic, then $g_{\mathrm{sym}}=$ $\left(\begin{array}{ll}0 & 1 \\ 1 & 0\end{array}\right) \in \hat{G}$. 
Proof. By construction, $\psi$ satisfies i) of Definition 6.1. Moreover, if (1) holds, then $\psi$ satisfies ii) of Definition 6.1 and, for any $V_{A, k}, V_{B, \ell} \in \mathfrak{h}_{\mathcal{Q}}$ we have that

$$
\begin{gathered}
{\left[V_{B, \ell}, \psi\left(\hat{V}_{A, k}\right)\right]+\left(\mathcal{L}_{V_{B, \ell}^{*}} \psi\right)\left(\hat{V}_{A, k}\right)=\left[V_{B, \ell}, V_{A, k}\right]-\psi\left(\left[V_{B, \ell}^{*}, V_{A, k}^{*}\right]\right)=} \\
=\left[V_{B, \ell}, V_{A, k}\right]-\psi\left(\left[V_{B, \ell}, V_{A, k}\right]^{*}\right)=0 .
\end{gathered}
$$

It follows that, if $\psi$ satisfies also (6.3),

$$
\left[V_{B, k}, \psi\left(\hat{\epsilon}_{i}\right)\right]+\left(\mathcal{L}_{V_{B, \ell}^{*}} \psi\right)(\hat{\epsilon})=\left[V_{B, k}, \epsilon_{i}\right]+d \psi\left(V_{B, k}^{*}, \hat{\epsilon}_{i}\right)=0 .
$$

So, for any element $\hat{X} \in\left\{\hat{\epsilon}_{i}, \hat{V}_{A, k}\right\}$, we have that $\left[V_{B, k}, \psi(\hat{X})\right]+\left(\mathcal{L}_{V_{B, k}^{*}} \psi\right)(\hat{X})=$ 0 . Since the vectors $\left\{\hat{\epsilon}_{i}, \hat{V}_{A, k}\right\}$ span $T_{u} P_{\mathrm{CM}}(M)$ at any point and, in the elliptic and hyperbolic case, the connected component of the identity $H_{\mathcal{Q}}^{o}$ coincides with $\exp \left(\mathfrak{h}_{\mathcal{Q}}\right)$, we conclude that $\psi$ is $H_{\mathcal{Q}}^{o}$-invariant. Finally, since $H_{\mathcal{Q}}=H_{\mathcal{Q}}^{o} \times\left\{e, g_{\mathrm{sym}}\right\}$, if also (3) is satisfied, then $\psi$ is invariant under the entire group $H_{\mathcal{Q}}$ and hence it is a Cartan connection.

The necessity of conditions (1) - (3) follows from the definitions.

Remark 6.3. Note that condition (6.3) can be written also as

$$
\left[V_{B, k}^{*}, \hat{\epsilon}_{i}\right]=\left[\widehat{V_{B, k}, \epsilon_{i}}\right] .
$$

Observe also that, by the proof of Proposition 5.1, if $M$ is hyperbolic, the element $g_{\text {sym }}$ maps any adapted coframe $\left(e^{1}, \ldots, e^{8}\right)$ into the adapted coframe $\left(e^{\prime 1}, \ldots, e^{\prime 8}\right)$ defined by

$$
\begin{gathered}
e^{\prime 1}=R_{g_{\mathrm{sym}}}^{*}\left(e^{2}\right), e^{\prime 2}=R_{g_{\mathrm{sym}}}^{*}\left(e^{1}\right) \\
e^{\prime 3}=R_{g_{\mathrm{sym}}}^{*}\left(e^{5}\right), e^{\prime 4}=R_{g_{\mathrm{sym}}}^{*}\left(e^{6}\right), e^{\prime 5}=R_{g_{\mathrm{sym}}}^{*}\left(e^{3}\right), e^{\prime 6}=R_{g_{\mathrm{sym}}}^{*}\left(e^{4}\right) .
\end{gathered}
$$

Similarly, by the same Proposition 5.1, if $M$ is elliptic, the element $g_{\text {sym }}$ maps any adapted coframe $\left(e^{1}, \ldots, e^{8}\right)$ into the adapted coframe $\left(e^{11}, \ldots, e^{\prime 8}\right)$ defined by

$$
E^{\prime 0}=R_{g_{\mathrm{sym}}}^{*}\left(\bar{E}^{0}\right), E^{\prime 1}=R_{g_{\mathrm{sym}}}^{*}\left(E^{2}\right), E^{\prime 2}=R_{g_{\mathrm{sym}}}^{*}\left(E^{1}\right)
$$

where, as before, $E^{0}=e^{1}+i e^{2}, E^{1}=e^{3}+i e^{4}$ and $E^{2}=e^{5}+i e^{6}$.

The next lemma shows that, locally, a Cartan connection always exists and that some additional useful properties can be always assumed.

Lemma 6.4. Let $\mathcal{U} \subset M$ be an open subset such that $\pi^{-1}(\mathcal{U}) \subset P_{\mathrm{CM}}(M)$ is trivializable. Then there exists a Cartan connection $\psi$ on $\pi^{-1}(\mathcal{U})$ modelled on $\left(\mathfrak{g}_{\mathcal{Q}}, \operatorname{Ad}\left(H_{\mathcal{Q}}\right)\right)$, such that

$$
\psi^{\epsilon_{i}}=\omega^{i},
$$

where the $\omega^{i}$ 's are the components of the tautological 1-form $\omega$ of $P_{\mathrm{CM}}(M)$.

Proof. It is known (see e.g. Ch. 5 in [17]) that any Cartan connection on $\pi^{-1}(\mathcal{U})$ can be constructed as follows. Let $\mu: \pi^{-1}(\mathcal{U}) \rightarrow \mathcal{U} \times H_{\mathcal{Q}}$ be a trivializing map and let $\delta_{x}$ be a family of linear maps $\delta_{x}: T_{x} \mathcal{U} \rightarrow \mathfrak{g}_{\mathcal{Q}}$, depending smoothly on the points $x \in \mathcal{U}$ and such that the compositions $p \circ \delta_{x}$ with the projection $p: \mathfrak{g}_{\mathcal{Q}} \rightarrow \mathfrak{g}_{\mathcal{Q}}^{-2}+\mathfrak{g}_{\mathcal{Q}}^{-1}$ are linear isomorphisms. If we denote by $\pi_{2}: \mathcal{U} \times H_{\mathcal{Q}} \rightarrow H_{\mathcal{Q}}$ the natural projection 
onto the second factor and we denote by $\omega_{H_{\mathcal{Q}}}$ the Maurer-Cartan form of $H_{\mathcal{Q}}$, then the 1 -form

$$
\psi_{\mu^{-1}(x, h)}=\mu^{*}\left(\operatorname{Ad}_{h^{-1}} \circ \delta_{x}+\pi_{2}^{*} \omega_{H_{\mathcal{Q}}}\right)
$$

is a Cartan connection. Moreover, any Cartan connection that is modelled on $\left(\mathfrak{g}_{\mathcal{Q}}, \operatorname{Ad}\left(H_{\mathcal{Q}}\right)\right)$ on $\pi^{-1}(\mathcal{U})$ is of the above form.

Consider now the family of linear maps

$$
\delta_{x}: T_{x} \mathcal{U} \rightarrow \mathfrak{g}_{\mathcal{Q}}^{-2}+\mathfrak{g}_{\mathcal{Q}}^{-1}, \quad \delta_{x}(X)=\sum \epsilon_{i} \omega_{\mu^{-1}(x, e)}^{i}\left(\mu_{*}^{-1}(X)\right)
$$

and the corresponding Cartan connection $\psi$ defined by (6.6). By construction, (6.5) holds at all points of the form $\mu^{-1}(x, e)$. By the $H_{\mathcal{Q}}$-invariance of $\psi$, the properties of the vectors $\epsilon_{i}$ and the transformation rules of the tautological 1-form $\omega$, it follows that the identity $(6.5)$ is satisfied at any point of $\pi^{-1}(\mathcal{U})$.

A (local) Cartan connection which satisfies (6.5) will be called good.

Consider a (local) good Cartan connection $\psi$ and let $\hat{\epsilon}_{i}$ and $\hat{V}_{A, k}$ be the associated fundamental vector fields. It is not hard to check that, if $\psi^{\prime}$ is a new $\mathfrak{g}_{\mathcal{Q}}$-valued 1-form, which satisfies (6.5) and conditions (i) and (ii) of Definition 6.1, then there exist some smooth $\mathbb{R}$-valued functions $S_{i}^{A, k}$ such that the fundamental vector fields $\hat{\epsilon}_{i}^{\prime}, \hat{V}_{A, k}^{\prime}$, determined by $\psi^{\prime}$, and the components $\psi^{\prime \epsilon_{i}}, \psi^{\prime V_{A, k}}$ of $\psi^{\prime}$ are as follows:

$$
\begin{gathered}
\hat{\epsilon}_{i}^{\prime}=\hat{\epsilon}_{i}+\sum_{A, k} S_{i}^{A, k} \hat{V}_{A, k}, \quad \hat{V}_{A, k}^{\prime}=\hat{V}_{A, k}=V_{A, k}^{*}, \\
\psi^{\prime \epsilon_{i}}=\omega^{i}, \quad \psi^{\prime V_{A, k}}=\psi^{V_{A, k}}-\sum_{i} S_{i}^{A, k} \omega^{i} .
\end{gathered}
$$

On the other hand, by Lemma 6.2 , this new $\mathfrak{g}_{\mathcal{Q}}$-valued 1 -form $\psi^{\prime}$ is a Cartan connection if and only if it is invariant under the element $g_{\mathrm{sym}} \in H_{\mathcal{Q}}$ and equation (6.3) is satisfied. This last condition means that for any element $V_{B, \ell}$ and any $\epsilon_{i}$ the following has to be satisfied:

$$
\begin{gathered}
{\left[V_{B, \ell}, \epsilon_{i}\right]=\sum_{j} \epsilon_{j} \omega^{j}\left(\left[V_{B, \ell}^{*}, \hat{\epsilon}_{i}^{\prime}\right]\right)+\sum_{C, m} V_{C, m} \psi^{\prime V_{C, m}}\left(\left[V_{B, \ell}^{*}, \hat{\epsilon}_{i}^{\prime}\right]\right)=} \\
=\sum_{j} \epsilon_{j} \omega^{j}\left(\left[V_{B, \ell}^{*}, \hat{\epsilon}_{i}\right]\right)+\sum_{j, A, k} \epsilon_{j} S_{i}^{A, k} \omega^{j}\left(\left[V_{B, \ell}^{*}, V_{A, k}^{*}\right]\right)+\sum_{j, A, k} \epsilon_{j} V_{B, \ell}^{*}\left(S_{i}^{A, k}\right) \omega^{j}\left(V_{A, k}^{*}\right)+ \\
+\sum_{C, m} V_{C, m}\left(\psi^{V_{C, m}}-\sum_{j} S_{j}^{C, m} \omega^{j}\right)\left(\left[V_{B, \ell}^{*}, \hat{\epsilon}_{i}+\sum_{A, k} S_{i}^{A, k} V_{A, k}^{*}\right]\right)= \\
=\sum_{j} \epsilon_{j} \omega^{j}\left(\left[V_{B, \ell}^{*}, \hat{\epsilon}_{i}\right]\right)+\sum_{C, m} V_{C, m} \psi^{V_{C, m}}\left(\left[V_{B, \ell}^{*}, \hat{\epsilon}_{i}\right]\right)+ \\
+\sum_{A, k, C, m} V_{C, m} V_{B, \ell}^{*}\left(S_{i}^{A, k}\right) \psi^{V_{C, m}}\left(V_{A, k}^{*}\right)+ \\
+\sum_{A, k, C, m} S_{i}^{A, k} V_{C, m} \psi^{V_{C, m}}\left(\left[V_{B, \ell}^{*}, V_{A, k}^{*}\right]\right)-\sum_{j, C, m} V_{C, m} S_{j}^{C, m} \omega^{j}\left(\left[V_{B, \ell}^{*}, \hat{\epsilon}_{i}\right]\right)
\end{gathered}
$$


Formula (6.9) simplifies considerably if we recall that $\psi$ is a Cartan connection (and hence satisfies (6.3)). In this way, we obtain that $\psi^{\prime}$ is a Cartan connection if and only if it is $g_{\mathrm{sym}}$-invariant and the functions $S_{i}^{A, k}$ satisfy, for any $V_{B, \ell}^{*}$

$$
V_{B, \ell}^{*}\left(S_{i}^{A, k}\right)=\sum_{j} S_{j}^{A, k} \omega^{j}\left(\left[V_{B, \ell}^{*}, \hat{\epsilon}_{i}\right]\right)-\sum_{C, m} S_{i}^{C, m} \psi^{V_{A, k}}\left(\left[V_{B, \ell}^{*}, V_{C, m}^{*}\right]\right)
$$

In the next two subsection, we will show that, for any good Cartan connection $\psi$, defined on a trivializable subset $\pi^{-1}(\mathcal{U}) \subset P_{\mathrm{CM}}(M)$, there exists a unique choice for a 1 -form $\psi^{\prime}$ presented as in (6.7) and (6.8) such that:

a) certain conditions on the values $d \omega^{i}\left(\hat{\epsilon}_{j}^{\prime}, \hat{\epsilon}_{k}^{\prime}\right)$ and $d \psi^{\prime V_{A, k}}\left(\hat{\epsilon}_{j}^{\prime}, \hat{\epsilon}_{k}^{\prime}\right)$ are satisfied;

b) $\psi^{\prime}$ is $g_{\text {sym }}$-invariant and satisfies (6.10) (hence it is a good Cartan connection).

The existence and uniqueness of such modification $\psi^{\prime}$ for any given good Cartan connection implies that it has to coincide on the overlaps of two trivializable sets $\pi^{-1}(\mathcal{U}), \pi^{-1}\left(\mathcal{U}^{\prime}\right)$, even if we started from two distinct good Cartan connections $\psi_{1}$ and $\psi_{2}$. For this reason all modifications $\psi^{\prime}$ can be patched together to defined a unique global good Cartan connection $\psi_{C M}$ on $P_{\mathrm{CM}}(M)$, which is necessarily canonical.

Finally, let us explain how it is possible to check if the canonical connection we obtain is the same (or, more precisely, equivalent) to the connection $\omega_{M}$ of Theorem 1.1. This can be done by means of Theorem 2.7 in [20], where the following necessary and sufficient conditions for the existence of an isomorphism between $\psi_{C M}$ and $\omega_{M}$ are given. Let us denote by $\left(\varepsilon_{A}\right), 1 \leq A \leq 6$, a basis for the subspace $\mathfrak{m} \stackrel{\text { def }}{=} \mathfrak{g}_{\mathcal{Q}}^{-2}+\mathfrak{g}_{\mathcal{Q}}^{-1} \subset \mathfrak{g}_{\mathcal{Q}}$ and let $\left(\varepsilon^{A}\right)$ a corresponding basis for $\mathfrak{g}_{\mathcal{Q}}^{1}+\mathfrak{g}_{\mathcal{Q}}^{2} \subset \mathfrak{g}_{\mathcal{Q}}$, which is dual w.r.t. the Cartan-Killing form $\mathcal{B}$ of $\mathfrak{g}_{\mathcal{Q}}$, i.e. such that $\mathcal{B}\left(\varepsilon^{A}, \varepsilon_{B}\right)=$ $\delta_{B}^{A}$. Then, in Theorem 2.7 in [20] it is given a necessary and sufficient condition, which may be rephrased for good Cartan connections saying that the following equations are satisfied for any $e_{B}$ (to obtain the following expression from the original statement in [20], we used the fact that for any $X \in \mathfrak{g}_{\mathcal{Q}}$ and any $\hat{e}^{A}$, $\left[\hat{e}_{A}, \psi_{C M}(\hat{X})\right]=-\mathcal{L}_{\hat{e}^{A}} \psi_{C M}(\hat{X})$ - see proof of Lemma 6.2$)$ :

$$
\sum_{A} \hat{\varepsilon}^{A}\left(d \psi_{C M}\left(\hat{\varepsilon}_{A}, \hat{\varepsilon}_{B}\right)\right)-\sum_{A} \frac{1}{2} d \psi_{C M}\left(\left[\varepsilon^{A}, \varepsilon_{B}\right]_{\mathfrak{m}}, \hat{\varepsilon}_{A}\right)=\sum_{A} \frac{1}{2}\left[\left[\varepsilon^{A}, \varepsilon_{B}\right]_{\mathfrak{m}}, \varepsilon_{A}\right],
$$

where $\left[\varepsilon^{A}, \varepsilon_{B}\right]_{\mathfrak{m}}$ denotes the natural projection of $\left[\varepsilon^{A}, \varepsilon_{B}\right]$ into the subspace $\mathfrak{m}=$ $\mathfrak{g}_{\mathcal{Q}}^{-2}+\mathfrak{g}_{\mathcal{Q}}^{-1}$.

If one consider a basis $\left(\varepsilon_{A}\right), 1 \leq A \leq 6$, given by the first six elements of the special basis for $\mathfrak{g}_{\mathcal{Q}}$ described in the Appendix, it is possible to realize that the corresponding dual basis $\left(\varepsilon^{A}\right)$ is given (up to factors) by the last six elements of the same basis for $\mathfrak{g}_{\mathcal{Q}}$. Then, using (6.12) and (6.13) below, it is possible to write down explicitly all components of the $\mathfrak{g}_{\mathcal{Q}}$-valued 1 -form on the left hand side of (6.11) and determine a set of conditions which is equivalent to (6.11). We will see that, among them, there are some of the which are not satisfied by a generic connection defined by the conditions mentioned in (a).

Let us now proceed with the construction of the canonical Cartan connection $\psi_{C M}$, following the steps (a) and (b) described above. 
Before going into the details of such a construction, we need the following technical fact, whose proof is just an application of definitions and of (6.3). In the following, most of the functions $S_{i}^{A, k}$ will coincide with functions which are linear combinations of the functions $d \omega^{k}\left(\hat{\epsilon}_{i}, \hat{\epsilon}_{j}\right)$ and $d \psi^{V_{C, 0}}\left(\hat{\epsilon}_{i}, \hat{\epsilon}_{j}\right)$. In order to check if (6.10) holds we need to evaluate the directional derivatives of such functions and they are given by the following expressions (we are assuming that $\psi$ is a Cartan connection):

$$
\begin{aligned}
& V_{B, m}^{*}\left(d \omega^{k}\left(\hat{\epsilon}_{i}, \hat{\epsilon}_{j}\right)\right)=d^{2} \omega^{k}\left(V_{B, m}^{*}, \hat{\epsilon}_{i}, \hat{\epsilon}_{j}\right)-\hat{\epsilon}_{i}\left(d \omega^{k}\left(\hat{\epsilon}_{j}, V_{B, m}^{*}\right)\right)-\hat{\epsilon}_{j}\left(d \omega^{k}\left(V_{B, m}^{*}, \hat{\epsilon}_{i}\right)\right)+ \\
& +d \omega^{k}\left(\left[V_{B, m}^{*}, \hat{\epsilon}_{i}\right], \hat{\epsilon}_{j}\right)+d \omega^{k}\left(\left[\hat{\epsilon}_{i}, \hat{\epsilon}_{j}\right], V_{B, m}^{*}\right)+d \omega^{k}\left(\left[\hat{\epsilon}_{j}, V_{B, m}^{*}\right], \hat{\epsilon}_{i}\right) \stackrel{(6.3)}{=} \\
& =d \omega^{k}\left(\left[V_{B, m}^{*}, \hat{\epsilon}_{i}\right], \hat{\epsilon}_{j}\right)+d \omega^{k}\left(\left[\hat{\epsilon}_{i}, \hat{\epsilon}_{j}\right], V_{B, m}^{*}\right)+d \omega^{k}\left(\left[\hat{\epsilon}_{j}, V_{B, m}^{*}\right], \hat{\epsilon}_{i}\right) \stackrel{(6.3)}{=} \\
& =d \omega^{k}\left(\left[\widehat{V_{B, m}, \epsilon_{i}}\right], \hat{\epsilon}_{j}\right)-d \omega^{k}\left(\left[\widehat{V_{B, m}, \epsilon_{j}}\right], \hat{\epsilon}_{i}\right)+\sum_{\ell} \omega^{\ell}\left(\left[\hat{\epsilon}_{i}, \hat{\epsilon}_{j}\right]\right) d \omega^{k}\left(\hat{\epsilon}_{\ell}, V_{B, m}^{*}\right)+ \\
& +\sum_{C, \ell} \psi^{V_{C, \ell}}\left(\left[\hat{\epsilon}_{i}, \hat{\epsilon}_{j}\right]\right) d \omega^{k}\left(V_{C, \ell}^{*}, V_{B, m}^{*}\right)= \\
& =d \omega^{k}\left(\left[\widehat{V_{B, m}, \epsilon_{i}}\right], \hat{\epsilon}_{j}\right)+d \omega^{k}\left(\hat{\epsilon}_{i},\left[\widehat{V_{B, m}, \epsilon_{j}}\right]\right)-\sum_{\ell} d \omega^{\ell}\left(\hat{\epsilon}_{i}, \hat{\epsilon}_{j}\right) \omega^{k}\left(\left[\widehat{V_{B, m}, \epsilon_{\ell}}\right]\right) \\
& V_{B, m}^{*}\left(d \psi^{V_{C, \ell}}\left(\hat{\epsilon}_{i}, \hat{\epsilon}_{j}\right)\right)= \\
& =d \psi^{V_{C, \ell}}\left(\left[V_{B, m}^{*}, \hat{\epsilon}_{i}\right], \hat{\epsilon}_{j}\right)+d \psi^{V_{C, \ell}}\left(\left[\hat{\epsilon}_{i}, \hat{\epsilon}_{j}\right], V_{B, m}^{*}\right)+d \psi^{V_{C, \ell}}\left(\left[\hat{\epsilon}_{j}, V_{B, m}^{*}\right], \hat{\epsilon}_{i}\right)=
\end{aligned}
$$

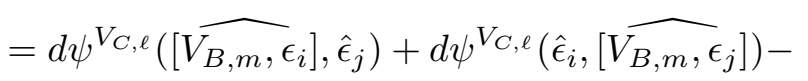

$$
\begin{aligned}
& -\sum_{n} d \omega^{n}\left(\hat{\epsilon}_{i}, \hat{\epsilon}_{j}\right) \psi^{V_{C, \ell}}\left(\left[\widehat{V_{B, m}, \epsilon_{n}}\right]\right)-\sum_{A, k} d \psi^{V_{A, k}}\left(\hat{\epsilon}_{i}, \hat{\epsilon}_{j}\right) \psi^{V_{C, \ell}}\left(\left[V_{B, m}, V_{A, k}\right]\right) \text {. }
\end{aligned}
$$

\subsection{Construction of a canonical Cartan connection on a hyperbolic manifold.}

Assume that $M$ is hyperbolic. In this case $\mathfrak{g}_{\mathcal{Q}} \simeq \mathfrak{s u}_{2,1} \oplus \mathfrak{s u}_{2,1}$ and the special basis $\left(\epsilon_{i}, V_{A, k}\right)$ is given in Appendix. Notice also that, using the same arguments which brought to (4.5), any good Cartan connection $\psi$ is so that

$$
\begin{array}{r}
d \omega^{1}=\omega^{3} \wedge \omega^{4}+S \omega^{5} \wedge \omega^{2}+T \omega^{6} \wedge \omega^{2}+2 \psi^{V_{2,1,0}} \wedge \omega^{1} \\
+ \text { linear combinations of }\left\{\omega^{1} \wedge \omega^{2}, \omega^{1} \wedge \omega^{j}, 3 \leq j \leq 6\right\} \\
d \omega^{2}=\omega^{5} \wedge \omega^{6}+S^{\prime} \omega^{3} \wedge \omega^{1}+T^{\prime} \omega^{4} \wedge \omega^{1}+2 \psi^{V_{2,2,0}} \wedge \omega^{2} \\
+ \text { linear combinations of }\left\{\omega^{1} \wedge \omega^{2}, \omega^{2} \wedge \omega^{j}, 3 \leq j \leq 6\right\}
\end{array}
$$

for some suitable functions $S, S^{\prime}, T, T^{\prime}$. Moreover, from the vanishing of

$$
d^{2} \omega^{1}\left(\hat{\epsilon}_{4}, \hat{\epsilon}_{5}, \hat{\epsilon}_{6}\right)=d^{2} \omega^{1}\left(\hat{\epsilon}_{3}, \hat{\epsilon}_{5}, \hat{\epsilon}_{6}\right)=d^{2} \omega^{2}\left(\hat{\epsilon}_{3}, \hat{\epsilon}_{4}, \hat{\epsilon}_{6}\right)=d^{2} \omega^{2}\left(\hat{\epsilon}_{3}, \hat{\epsilon}_{4}, \hat{\epsilon}_{5}\right)=0
$$

and using (6.3), one can check that for any good Cartan connection $\psi$ the following identities hold:

$$
d \omega^{3}\left(\hat{\epsilon}_{5}, \hat{\epsilon}_{6}\right)=d \omega^{4}\left(\hat{\epsilon}_{5}, \hat{\epsilon}_{6}\right)=d \omega^{5}\left(\hat{\epsilon}_{3}, \hat{\epsilon}_{4}\right)=d \omega^{6}\left(\hat{\epsilon}_{3}, \hat{\epsilon}_{4}\right)=0 .
$$

Now we will proceed with the construction of a canonical Cartan connection, which is based on a sequence of technical lemmata. 
Lemma 6.5. On any trivializable open subset $\pi^{-1}(\mathcal{U}) \subset P_{\mathrm{CM}}(M)$, there exists a good Cartan connections $\psi$, which satisfies the following conditions for any $a=1,2$, $j=1,3,4$ and $k=2,5,6$ :

$$
\begin{gathered}
d \omega^{1}\left(\hat{\epsilon}_{1}, \hat{\epsilon}_{3}\right)=d \omega^{1}\left(\hat{\epsilon}_{1}, \hat{\epsilon}_{4}\right)=d \omega^{2}\left(\hat{\epsilon}_{2}, \hat{\epsilon}_{5}\right)=d \omega^{2}\left(\hat{\epsilon}_{2}, \hat{\epsilon}_{6}\right)=0, \\
d \omega^{3}\left(\hat{\epsilon}_{3}, \hat{\epsilon}_{4}\right)=d \omega^{4}\left(\hat{\epsilon}_{3}, \hat{\epsilon}_{4}\right)=d \omega^{5}\left(\hat{\epsilon}_{5}, \hat{\epsilon}_{6}\right)=d \omega^{6}\left(\hat{\epsilon}_{5}, \hat{\epsilon}_{6}\right)=0, \\
d \omega^{6}\left(\hat{\epsilon}_{j}, \hat{\epsilon}_{5}\right)-d \omega^{5}\left(\hat{\epsilon}_{j}, \hat{\epsilon}_{6}\right)=d \omega^{5}\left(\hat{\epsilon}_{j}, \hat{\epsilon}_{5}\right)+d \omega^{6}\left(\hat{\epsilon}_{j}, \hat{\epsilon}_{6}\right)+d \omega^{2}\left(\hat{\epsilon}_{j}, \hat{\epsilon}_{2}\right)=0, \\
d \omega^{4}\left(\hat{\epsilon}_{k}, \hat{\epsilon}_{3}\right)-d \omega^{3}\left(\hat{\epsilon}_{k}, \hat{\epsilon}_{4}\right)=d \omega^{3}\left(\hat{\epsilon}_{k}, \hat{\epsilon}_{3}\right)+d \omega^{4}\left(\hat{\epsilon}_{k}, \hat{\epsilon}_{4}\right)+d \omega^{1}\left(\hat{\epsilon}_{k}, \hat{\epsilon}_{1}\right)=0 \\
d \omega^{3}\left(\hat{\epsilon}_{3}, \hat{\epsilon}_{1}\right)=d \omega^{4}\left(\hat{\epsilon}_{4}, \hat{\epsilon}_{1}\right)=d \omega^{3}\left(\hat{\epsilon}_{4}, \hat{\epsilon}_{1}\right)=d \omega^{4}\left(\hat{\epsilon}_{3}, \hat{\epsilon}_{1}\right)=0 \\
d \omega^{5}\left(\hat{\epsilon}_{5}, \hat{\epsilon}_{2}\right)=d \omega^{6}\left(\hat{\epsilon}_{6}, \hat{\epsilon}_{2}\right)=d \omega^{5}\left(\hat{\epsilon}_{6}, \hat{\epsilon}_{2}\right)=d \omega^{6}\left(\hat{\epsilon}_{5}, \hat{\epsilon}_{2}\right)=0 .
\end{gathered}
$$

Moreover, if $\psi^{\prime}$ is another good Cartan connection with the same properties and expressed in terms of $\psi$ as in (6.7), (6.8), then the functions $S_{J}^{a, b, 0}, S_{1}^{2,2,0}, S_{2}^{2,1,0}$, with $a, b,=1,2$ and $3 \leq J \leq 6$, are vanishing, while the others satisfy the following linear relations:

$$
\begin{aligned}
& S_{1}^{1,1,0}=S_{4}^{2,1,1}=-S_{3}^{1,1,1}, \quad S_{1}^{2,1,0}=S_{3}^{2,1,1}=S_{4}^{1,1,1}, \\
& S_{2}^{1,2,0}=S_{6}^{2,2,1}=-S_{5}^{1,2,1}, \quad S_{2}^{2,2,0}=S_{5}^{2,2,1}=S_{6}^{1,2,1} .
\end{aligned}
$$

Finally, for any good Cartan connection $\psi$, which satisfies (6.17) - (6.21), the following identities hold for any $a=1,2, j=1,3,4$ and $k=2,5,6$ :

$$
d \omega^{2}\left(\hat{\epsilon}_{2}, \hat{\epsilon}_{j}\right)=d \omega^{1}\left(\hat{\epsilon}_{1}, \hat{\epsilon}_{k}\right)=d \psi^{V_{2, a, 0}}\left(\hat{\epsilon}_{3}, \hat{\epsilon}_{4}\right)=d \psi^{V_{2, a, 0}}\left(\hat{\epsilon}_{5}, \hat{\epsilon}_{6}\right)=0 .
$$

Proof. Consider a good Cartan connection $\psi$ on $\pi^{-1}(\mathcal{U}) \subset P_{\mathrm{CM}}(M)$ and let $\psi^{\prime}$ be another $\mathfrak{g}_{\mathcal{Q}}$-valued 1-form defined as in (6.7) and (6.8) by means of some functions $S_{i}^{a, b, k}$. The 1 -form $\psi^{\prime}$ satisfies $(6.17)_{1}$ if and only if

$$
\begin{gathered}
0=d \omega^{1}\left(\hat{\epsilon}_{1}^{\prime}, \hat{\epsilon}_{3}^{\prime}\right)=-\omega^{1}\left(\left[\hat{\epsilon}_{1}+\sum_{b, c, k} S_{1}^{b, c, k} V_{b, c, k}^{*}, \hat{\epsilon}_{3}+\sum_{d, e, \ell} S_{3}^{d, e, \ell} V_{d, e, \ell}^{*}\right]\right)= \\
=-\omega^{1}\left(\left[\hat{\epsilon}_{1}, \hat{\epsilon}_{3}\right]\right)+S_{3}^{2, a, 0} \omega^{1}\left(\left[V_{2, a, 0}^{*}, \hat{\epsilon}_{1}\right]\right)= \\
=d \omega^{1}\left(\hat{\epsilon}_{1}, \hat{\epsilon}_{3}\right)-2 S_{3}^{2,1,0},
\end{gathered}
$$

i.e. $S_{3}^{2,1,0}=-\frac{1}{2} d \omega^{1}\left(\hat{\epsilon}_{3}, \hat{\epsilon}_{1}\right)$. In a similar way, one can check that the other conditions in (6.17) and (6.18) are satisfied if and only if

$$
\begin{aligned}
S_{4}^{2,1,0} & =-\frac{1}{2} d \omega^{1}\left(\hat{\epsilon}_{4}, \hat{\epsilon}_{1}\right), \quad S_{5}^{2,2,0}=-\frac{1}{2} d \omega^{2}\left(\hat{\epsilon}_{5}, \hat{\epsilon}_{2}\right), \quad S_{6}^{2,2,0}=-\frac{1}{2} d \omega^{2}\left(\hat{\epsilon}_{6}, \hat{\epsilon}_{2}\right), \\
S_{3}^{1,1,0} & =-d \omega^{3}\left(\hat{\epsilon}_{3}, \hat{\epsilon}_{4}\right)-\frac{1}{2} d \omega^{1}\left(\hat{\epsilon}_{4}, \hat{\epsilon}_{1}\right), \quad S_{4}^{1,1,0}=-d \omega^{4}\left(\hat{\epsilon}_{3}, \hat{\epsilon}_{4}\right)+\frac{1}{2} d \omega^{1}\left(\hat{\epsilon}_{3}, \hat{\epsilon}_{1}\right), \\
S_{j}^{1,2,0} & =\frac{1}{2}\left(d \omega^{6}\left(\hat{\epsilon}_{j}, \hat{\epsilon}_{5}\right)-d \omega^{5}\left(\hat{\epsilon}_{j}, \hat{\epsilon}_{6}\right)\right), S_{j}^{1,2,0}=\frac{1}{2}\left(d \omega^{4}\left(\hat{\epsilon}_{k}, \hat{\epsilon}_{3}\right)-d \omega^{3}\left(\hat{\epsilon}_{k}, \hat{\epsilon}_{4}\right)\right),
\end{aligned}
$$




$$
\begin{aligned}
S_{j}^{2,2,0} & =-\frac{1}{4}\left(d \omega^{5}\left(\hat{\epsilon}_{j}, \hat{\epsilon}_{5}\right)+d \omega^{6}\left(\hat{\epsilon}_{j}, \hat{\epsilon}_{6}\right)+d \omega^{2}\left(\hat{\epsilon}_{j}, \hat{\epsilon}_{2}\right)\right) \\
S_{k}^{2,1,0} & =-\frac{1}{4}\left(d \omega^{3}\left(\hat{\epsilon}_{k}, \hat{\epsilon}_{3}\right)+d \omega^{4}\left(\hat{\epsilon}_{k}, \hat{\epsilon}_{4}\right)+d \omega^{1}\left(\hat{\epsilon}_{k}, \hat{\epsilon}_{1}\right)\right)
\end{aligned}
$$

for any $j=1,3,4$ and $k=2,5,6$.

A tedious but straightforward check, based on (6.12), shows that if we choose the functions $S_{J}^{A, k}$ so that (6.27)- (6.31) hold and all the remaining functions so that (6.10) is satisfied, then $\psi^{\prime}$ satisfies (6.3) and condition (3) of Lemma 6.2. This means that $\psi^{\prime}$ is a Cartan connection and hence that we may assume that $\psi$ satisfies also (6.17) - (6.21) (by replacing $\psi$ with $\left.\psi^{\prime}\right)$. From $(6.26)-(6.31)$, it is clear that any other $\psi^{\prime}$ with such properties is defined by functions $S_{J}^{A, k}$ so that $S_{1}^{a, 2,0}=S_{2}^{a, 1,0}=S_{J}^{a, b, 0}=0$, for $a, b=1,2$ and $3 \leq J \leq 6$.

Let us now look for those $\psi^{\prime}$ which satisfy also (6.21) and (6.22), we see that this occurs if and only if the following holds:

$$
\begin{aligned}
& S_{1}^{2,1,0}-S_{3}^{2,1,1}=d \omega^{3}\left(\hat{\epsilon}_{3}, \hat{\epsilon}_{1}\right), S_{1}^{1,1,0}-S_{4}^{2,1,1}=d \omega^{3}\left(\hat{\epsilon}_{4}, \hat{\epsilon}_{1}\right), \\
& S_{1}^{1,1,0}+S_{3}^{1,1,1}=d \omega^{4}\left(\hat{\epsilon}_{1}, \hat{\epsilon}_{3}\right), S_{1}^{2,1,0}-S_{4}^{1,1,1}=d \omega^{4}\left(\hat{\epsilon}_{4}, \hat{\epsilon}_{1}\right), \\
& S_{2}^{2,2,0}-S_{5}^{2,2,1}=d \omega^{5}\left(\hat{\epsilon}_{5}, \hat{\epsilon}_{2}\right), S_{2}^{1,2,0}-S_{6}^{2,2,1}=d \omega^{5}\left(\hat{\epsilon}_{6}, \hat{\epsilon}_{2}\right), \\
& S_{2}^{1,2,0}+S_{5}^{1,2,1}=d \omega^{6}\left(\hat{\epsilon}_{2}, \hat{\epsilon}_{5}\right), S_{2}^{2,2,0}-S_{6}^{1,2,1}=d \omega^{6}\left(\hat{\epsilon}_{6}, \hat{\epsilon}_{2}\right) .
\end{aligned}
$$

It is not hard to realize that the system given by the equations (6.32) -(6.35) has maximal rank and it is therefore solvable at any point. Moreover, with some other straightforward computations, it can be checked that any set of functions which satisfy (6.32) - (6.35) at the points of a submanifold transversal to the fibers can be smoothly extended to functions which satisfy (6.10) and condition (3) of Lemma 6.2 and which at the same time are solutions of the above given system of equations. From this we get the existence of a Cartan $\psi$ which satisfies also (6.21) and (6.22). The second claim is a direct consequence of (6.26) - (6.35). The last claim can be checked by evaluating $d^{2} \omega^{2}\left(\hat{\epsilon}_{j}, \hat{\epsilon}_{5}, \hat{\epsilon}_{6}\right), d^{2} \omega^{1}\left(\hat{\epsilon}_{k}, \hat{\epsilon}_{3}, \hat{\epsilon}_{4}\right), d^{2} \omega^{a}\left(\hat{\epsilon}_{a}, \hat{\epsilon}_{3}, \hat{\epsilon}_{4}\right)$ and $d^{2} \omega^{a}\left(\hat{\epsilon}_{a}, \hat{\epsilon}_{5}, \hat{\epsilon}_{6}\right)$ with $a=1,2, j=1,3,4, k=2,5,6$, and recalling that they have to vanish because of the well-known triviality of the operator $d^{2}$.

Lemma 6.6. On any trivializable open subset $\pi^{-1}(\mathcal{U}) \subset P_{\mathrm{CM}}(M)$, there exists a good Cartan connection $\psi$, which satisfies (6.17) - (6.21) and the conditions:

$$
\begin{gathered}
d \psi^{V_{1,1,0}}\left(\hat{\epsilon}_{3}, \hat{\epsilon}_{4}\right)=d \psi^{V_{1,2,0}}\left(\hat{\epsilon}_{5}, \hat{\epsilon}_{6}\right)=0, \\
d \psi^{V_{1,1,0}}\left(\hat{\epsilon}_{3}, \hat{\epsilon}_{1}\right)+d \psi^{V_{2,1,0}}\left(\hat{\epsilon}_{4}, \hat{\epsilon}_{1}\right)=d \psi^{V_{2,1,0}}\left(\hat{\epsilon}_{3}, \hat{\epsilon}_{1}\right)-d \psi^{V_{1,1,0}}\left(\hat{\epsilon}_{4}, \hat{\epsilon}_{1}\right)=0, \\
d \psi^{V_{1,2,0}}\left(\hat{\epsilon}_{5}, \hat{\epsilon}_{2}\right)+d \psi^{V_{2,2,0}}\left(\hat{\epsilon}_{6}, \hat{\epsilon}_{2}\right)=d \psi^{V_{2,2,0}}\left(\hat{\epsilon}_{5}, \hat{\epsilon}_{2}\right)-d \psi^{V_{1,1,0}}\left(\hat{\epsilon}_{6}, \hat{\epsilon}_{2}\right)=0,
\end{gathered}
$$

Moreover, if $\psi^{\prime}$ is another good Cartan connection with the same properties and expressed in terms of $\psi$ as in (6.7), (6.8), then all functions $S_{J}^{A, k}$ are vanishing except the functions which appear in $(6.23)_{2},(6.24)_{2}$ and the functions $S_{a}^{c, a, 1}, S_{j}^{a, 2,1}$, $S_{k}^{a, 1,1}$ and $S_{J}^{a, b, 2}$ with $a=1,2, j=3,4, k=5,6$ and $1 \leq J \leq 6$, among which the following relations have to be satisfied:

$$
S_{1}^{1,1,1}=-\frac{1}{2} S_{3}^{1,1,2}, \quad S_{1}^{2,1,1}=\frac{1}{2} S_{4}^{1,1,2}, \quad S_{2}^{1,2,1}=-\frac{1}{2} S_{5}^{1,2,2}, \quad S_{2}^{2,2,1}=\frac{1}{2} S_{6}^{1,2,2},
$$


Proof. Starting as in the previous lemma, let us fix a good Cartan connection $\psi$ on $\pi^{-1}(\mathcal{U}) \subset P_{\mathrm{CM}}(M)$, which satisfies Proposition 6.5 , and let $\psi^{\prime}$ be an arbitrary $\mathfrak{g}_{\mathcal{Q}^{-}}$ valued 1 -form, defined as in (6.8) by means of some functions $S_{i}^{a, b, k}$, which satisfies the same conditions. From the results of the previous lemma on the functions $S_{i}^{a, b, k}$ and by the same line of arguments used in its proof, we see that $(6.36)_{1}$ is satisfied if and only if

$$
\begin{gathered}
0=d \psi^{V_{1,1,0}}\left(\hat{\epsilon}_{3}, \hat{\epsilon}_{4}\right)-\sum_{i=1}^{6} S_{i}^{1,1,0} d \omega^{i}\left(\hat{\epsilon}_{3}, \hat{\epsilon}_{4}\right)-\frac{3}{2} S_{4}^{2,1,1}+\frac{3}{2} S_{3}^{1,1,1}= \\
=d \psi^{V_{1,1,0}}\left(\hat{\epsilon}_{3}, \hat{\epsilon}_{4}\right)-S_{1}^{1,1,0}-\frac{3}{2} S_{4}^{2,1,1}+\frac{3}{2} S_{3}^{1,1,1}
\end{gathered}
$$

and hence, by (6.23), if and only if

$$
S_{1}^{1,1,0}=S_{4}^{2,1,1}=-S_{3}^{1,1,1}=\frac{1}{4} d \psi^{V_{1,1,0}}\left(\hat{\epsilon}_{3}, \hat{\epsilon}_{4}\right) .
$$

Similarly, the other equality of (6.36) is true if and only if

$$
S_{2}^{1,2,0}=S_{5}^{2,2,1}=-S_{6}^{1,1,1}=\frac{1}{4} d \psi^{V_{1,2,0}}\left(\hat{\epsilon}_{5}, \hat{\epsilon}_{6}\right) .
$$

With similar arguments we get also that (6.37) - (6.42) are satisfied if and only if

$$
\begin{aligned}
& 2 S_{1}^{2,1,1}-S_{4}^{1,1,2}=d \psi^{V_{1,1,0}}\left(\hat{\epsilon}_{3}, \hat{\epsilon}_{1}\right)+d \psi^{V_{2,1,0}}\left(\hat{\epsilon}_{4}, \hat{\epsilon}_{1}\right), \\
& 2 S_{1}^{1,1,1}+S_{3}^{1,1,2}=d \psi^{V_{1,1,0}}\left(\hat{\epsilon}_{4}, \hat{\epsilon}_{1}\right)-d \psi^{V_{2,1,0}}\left(\hat{\epsilon}_{3}, \hat{\epsilon}_{1}\right), \\
& 2 S_{2}^{2,2,1}-S_{6}^{1,2,2}=d \psi^{V_{2,2,0}}\left(\hat{\epsilon}_{5}, \hat{\epsilon}_{2}\right)+d \psi^{V_{1,2,0}}\left(\hat{\epsilon}_{6}, \hat{\epsilon}_{2}\right), \\
& 2 S_{2}^{1,2,1}+S_{5}^{1,2,2}=d \psi^{V_{1,2,0}}\left(\hat{\epsilon}_{6}, \hat{\epsilon}_{2}\right)-d \psi^{V_{2,2,0}}\left(\hat{\epsilon}_{5}, \hat{\epsilon}_{2}\right),
\end{aligned}
$$

The proofs of (6.42) - (6.45) are slightly more involved than those in the previous lemma and so, for convenience of the reader, we exhibit the steps which bring to (6.42) (the other conditions are obtained in a very similar way). To check that, it is enough to observe that $\psi^{\prime}$ satisfies $(6.37)_{1}$ if and only if

$$
\begin{aligned}
& 0=-\psi^{\prime V_{1,1,0}}\left(\left[\hat{\epsilon}_{3}^{\prime}, \hat{\epsilon}_{1}^{\prime}\right]\right)-\psi^{\prime V_{2,1,0}}\left(\left[\hat{\epsilon}_{4}^{\prime}, \hat{\epsilon}_{1}^{\prime}\right]\right)= \\
& =-\psi^{V_{1,1,0}}\left(\left[\hat{\epsilon}_{3}^{\prime}, \hat{\epsilon}_{1}^{\prime}\right]\right)-\psi^{\prime V_{2,1,0}}\left(\left[\hat{\epsilon}_{4}^{\prime}, \hat{\epsilon}_{1}^{\prime}\right]\right)+\sum_{j=1}^{6}\left(S_{j}^{1,1,0} \omega^{j}\left(\left[\hat{\epsilon}_{3}^{\prime}, \hat{\epsilon}_{1}^{\prime}\right]\right)+S_{j}^{2,1,0} \omega^{j}\left(\left[\hat{\epsilon}_{4}^{\prime}, \hat{\epsilon}_{1}^{\prime}\right]\right)\right)= \\
& =d \psi^{V_{1,1,0}}\left(\left[\hat{\epsilon}_{3}, \hat{\epsilon}_{1}\right]\right)+d \psi^{V_{2,1,0}}\left(\left[\hat{\epsilon}_{4}, \hat{\epsilon}_{1}\right]\right)-2 S_{1}^{2,1,1}+S_{4}^{1,1,2}- \\
& -S_{1}^{1,1,0} d \omega^{1}\left(\hat{\epsilon}_{3}^{\prime}, \hat{\epsilon}_{1}^{\prime}\right)-S_{1}^{2,1,0} d \omega^{1}\left(\hat{\epsilon}_{4}^{\prime}, \hat{\epsilon}_{1}^{\prime}\right)= \\
& =d \psi^{V_{1,1,0}}\left(\left[\hat{\epsilon}_{3}, \hat{\epsilon}_{1}\right]\right)+d \psi^{V_{2,1,0}}\left(\left[\hat{\epsilon}_{4}, \hat{\epsilon}_{1}\right]\right)-2 S_{1}^{2,1,1}+S_{4}^{1,1,2},
\end{aligned}
$$

where we used (6.17) and the claim on the vanishing of certain functions $S_{j}^{1,1,0}$ given in Lemma 6.5. 
Now, it is clear that, even if (6.42) - (6.45) do not determine completely the functions $S_{a}^{1, a, 1}, S_{a+2}^{1, a, 2}$ and $S_{a+3}^{1, a, 2}, a=1,2$, they form a system of maximal rank, which always admits a solution. As in the proof of the previous lemma, we may choose the functions $S_{J}^{A, k}$ so that all conditions (6.40) - (6.47) are satisfied at the points of a submanifold which is transversal to all fibers and then, find out what are the conditions in order to be able to extend these functions to the whole trivializable open set, so that (6.10) and (3) of Lemma 6.2 are true and obtain in this way a good Cartan connection. By some straightforward computations, based on (6.12), (6.13) and the hypothesis, we get that all this can be done implying that a Cartan connection $\psi$ which satisfies all requirements exists and that for any other Cartan connection $\psi^{\prime}$ the functions $S_{J}^{A, k}$ satisfy the relations (6.39).

Lemma 6.7. On any trivializable open subset $\pi^{-1}(\mathcal{U}) \subset P_{\mathrm{CM}}(M)$, there exists a unique good Cartan connection $\psi$, which satisfies (6.17) - (6.22), (6.36) - (6.38) and the following conditions for any $a=1,2, j=1,3,4$ and $k=2,5,6$ :

$$
\begin{gathered}
d \psi^{V_{a, 1,1}}\left(\hat{\epsilon}_{3}, \hat{\epsilon}_{4}\right)=d \psi^{V_{a, 2,1}}\left(\hat{\epsilon}_{5}, \hat{\epsilon}_{6}\right)=0 \\
d \omega^{6}\left(\hat{\epsilon}_{j}, \hat{\epsilon}_{2}\right)+d \psi^{V_{1,2,0}}\left(\hat{\epsilon}_{j}, \hat{\epsilon}_{6}\right)-d \psi^{V_{2,2,0}}\left(\hat{\epsilon}_{j}, \hat{\epsilon}_{5}\right)=0, \\
d \omega^{5}\left(\hat{\epsilon}_{j}, \hat{\epsilon}_{2}\right)+d \psi^{V_{1,2,0}}\left(\hat{\epsilon}_{j}, \hat{\epsilon}_{5}\right)+d \psi^{V_{2,2,0}}\left(\hat{\epsilon}_{j}, \hat{\epsilon}_{6}\right)=0 \\
d \omega^{4}\left(\hat{\epsilon}_{k}, \hat{\epsilon}_{1}\right)+d \psi^{V_{1,1,0}}\left(\hat{\epsilon}_{k}, \hat{\epsilon}_{4}\right)-d \psi^{V_{2,1,0}}\left(\hat{\epsilon}_{k}, \hat{\epsilon}_{3}\right)=0 \\
d \omega^{3}\left(\hat{\epsilon}_{k}, \hat{\epsilon}_{1}\right)+d \psi^{V_{1,1,0}}\left(\hat{\epsilon}_{k}, \hat{\epsilon}_{3}\right)+d \psi^{V_{2,1,0}}\left(\hat{\epsilon}_{k}, \hat{\epsilon}_{4}\right)=0 \\
d \psi^{V_{1,1,2}}\left(\hat{\epsilon}_{3}, \hat{\epsilon}_{4}\right)=d \psi^{V_{1,2,2}}\left(\hat{\epsilon}_{5}, \hat{\epsilon}_{6}\right)=0 \\
2 d \psi^{V_{2,2,0}}\left(\hat{\epsilon}_{j}, \hat{\epsilon}_{2}\right)+d \psi^{V_{2,2,1}}\left(\hat{\epsilon}_{j}, \hat{\epsilon}_{5}\right)+d \psi^{V_{1,2,1}}\left(\hat{\epsilon}_{j}, \hat{\epsilon}_{6}\right)=0 \\
2 d \psi^{V_{2,1,0}}\left(\hat{\epsilon}_{k}, \hat{\epsilon}_{1}\right)+d \psi^{V_{2,1,1}}\left(\hat{\epsilon}_{k}, \hat{\epsilon}_{3}\right)+d \psi^{V_{1,1,1}}\left(\hat{\epsilon}_{k}, \hat{\epsilon}_{4}\right)=0 .
\end{gathered}
$$

Proof. The proof proceeds exactly as for the previous two lemmata. Assuming that $\psi$ satisfies all claims of Lemma 6.6 and 6.7, let us look for a modified $\psi^{\prime}$ which has the same properties and for which $(6.46)_{1}$ hold with $a=1$. We get that the functions $S_{i}^{A, k}$ have to satisfy

$$
\begin{gathered}
0=d \psi^{\prime V_{1,1,1}}\left(\hat{\epsilon}_{3}^{\prime}, \hat{\epsilon}_{4}^{\prime}\right)=-\psi^{V_{1,1,1}}\left(\left[\hat{\epsilon}_{3}^{\prime}, \hat{\epsilon}_{4}^{\prime}\right]\right)-\sum_{j=1}^{6} S_{j}^{1,1,1} d \omega^{j}\left(\hat{\epsilon}_{3}^{\prime}, \hat{\epsilon}_{4}^{\prime}\right)= \\
=-\psi^{V_{1,1,1}}\left(\left[\hat{\epsilon}_{3}, \hat{\epsilon}_{4}\right]\right)+S_{3}^{1,1,2}-S_{1}^{1,1,1}+\sum_{k=5,6} S_{k}^{1,1,1} d d \omega^{k}\left(\hat{\epsilon}_{3}, \hat{\epsilon}_{4}\right)= \\
=d \psi^{V_{1,1,1}}\left(\hat{\epsilon}_{3}, \hat{\epsilon}_{4}\right)+\frac{3}{2} S_{3}^{1,1,2}+\sum_{k=5,6} S_{k}^{1,1,1} d \omega^{k}\left(\hat{\epsilon}_{3}, \hat{\epsilon}_{4}\right),
\end{gathered}
$$

where we used the fact that $S_{j}^{1,1,1}=0$ when $j=3,4$ and that $d \omega^{2}\left(\hat{\epsilon}_{3}, \hat{\epsilon}_{4}\right)=0$. So we have that

$$
S_{3}^{1,1,2}=-\frac{2}{3} d \psi^{V_{1,1,1}}\left(\hat{\epsilon}_{3}, \hat{\epsilon}_{4}\right)+\frac{2}{3} \sum_{k=5,6} S_{k}^{1,1,1} d \omega^{k}\left(\hat{\epsilon}_{3}, \hat{\epsilon}_{4}\right)
$$


On the other hand, for any $\psi^{\prime}$ which is also a Cartan connection, we have to impose that (6.10) holds true. This implies that for any $k=5,6$

$$
V_{1,1,1}^{*}\left(S_{3}^{1,2,2}\right)=-S_{3}^{2,1,1}, \quad V_{1,1,1}^{*}\left(S_{k}^{1,1,1}\right)=S_{k}^{2,1,0}=0
$$

where we use the fact that $S_{k}^{2,1,0}=0$ by Lemma 6.5. So, from (6.54) and using (6.12) and (6.13), we get that any $\psi^{\prime}$ which satisfies $(6.46)_{1}$ and (6.10) has to satisfies also

$$
\begin{aligned}
& S_{1}^{2,1,0}=S_{4}^{1,1,1}=S_{3}^{2,1,1}=-V_{1,1,1}^{*}\left(S_{3}^{1,1,2}\right)= \\
& =-V_{1,1,1}^{*}\left(-\frac{2}{3} d \psi^{V_{1,1,1}}\left(\hat{\epsilon}_{3}, \hat{\epsilon}_{4}\right)+\frac{2}{3} \sum_{k=5,6} S_{k}^{1,1,1} d d \omega^{k}\left(\hat{\epsilon}_{3}, \hat{\epsilon}_{4}\right)\right)= \\
& =\frac{2}{3} d \psi^{V_{2,1,0}}\left(\hat{\epsilon}_{3}, \hat{\epsilon}_{4}\right)-\frac{2}{3} \sum_{k=5,6} S_{k}^{1,1,1} d \omega^{2}\left(\hat{\epsilon}_{3}, \hat{\epsilon}_{4}\right)=0 .
\end{aligned}
$$

With similar arguments applied to $(6.46)_{2}$ we get that

$$
S_{2}^{2,2,0}=S_{6}^{1,2,1}=S_{5}^{2,2,1}=0 .
$$

Now, using (6.55) and (6.56), we may determine the conditions for which (6.47) (6.50) are satisfied. With the same arguments of before we find that we must have

$$
\begin{aligned}
& S_{j}^{1,2,1}=-\frac{1}{3}\left(d \omega^{6}\left(\hat{\epsilon}_{j}, \hat{\epsilon}_{2}\right)+d \psi^{V_{1,2,0}}\left(\hat{\epsilon}_{j}, \hat{\epsilon}_{6}\right)-d \psi^{V_{2,2,0}}\left(\hat{\epsilon}_{j}, \hat{\epsilon}_{5}\right)\right), \\
& S_{j}^{2,2,1}=-\frac{1}{3}\left(d \omega^{5}\left(\hat{\epsilon}_{j}, \hat{\epsilon}_{2}\right)+d \psi^{V_{1,2,0}}\left(\hat{\epsilon}_{j}, \hat{\epsilon}_{5}\right)+d \psi^{V_{2,2,0}}\left(\hat{\epsilon}_{j}, \hat{\epsilon}_{6}\right)\right), \\
& S_{k}^{1,1,1}=-\frac{1}{3}\left(d \omega^{4}\left(\hat{\epsilon}_{k}, \hat{\epsilon}_{1}\right)+d \psi^{V_{1,1,0}}\left(\hat{\epsilon}_{k}, \hat{\epsilon}_{4}\right)-d \psi^{V_{2,1,0}}\left(\hat{\epsilon}_{k}, \hat{\epsilon}_{3}\right)\right), \\
& S_{k}^{2,1,1}=-\frac{1}{3}\left(d \omega^{3}\left(\hat{\epsilon}_{k}, \hat{\epsilon}_{1}\right)+d \psi^{V_{1,1,0}}\left(\hat{\epsilon}_{k}, \hat{\epsilon}_{3}\right)+d \psi^{V_{2,1,0}}\left(\hat{\epsilon}_{k}, \hat{\epsilon}_{4}\right)\right),
\end{aligned}
$$

for any $j=1,3,4$ and $k=2,5,6$. As before, a tedious but straightforward check shows that functions which satisfy $(6.55)$ - (6.60) define a $\psi^{\prime}$ so that all equations (6.10) and Lemma $6.2(3)$ are satisfied. So, we may replace $\psi$ with $\psi^{\prime}$ and assume that also the conditions (6.48) - (6.50) are true. Moreover, from the previous discussion, we see that, if we look for another $\psi^{\prime}$ which satisfies those conditions and so that (6.46) and (6.47) are true, we have to look among the $\mathfrak{g}_{\mathcal{Q}}$-valued 1forms for which the functions $S_{1}^{2,1,0}$ and $S_{2}^{2,2,0}$ are vanishing, by (6.55) and (6.56). Moreover, by (6.57) - (6.60), we also have to suppose that all functions $S_{j}^{a, 2,1}$ and $S_{k}^{a, 1,1}$, with $j=1,3,4$ and $k=2,5,6$ are vanishing. With these assumptions, we get that (6.46) and (6.47) are true if and only if

$$
\begin{gathered}
S_{3}^{1,1,2}=-\frac{2}{3} d \psi^{V_{1,1,1}}\left(\hat{\epsilon}_{3}, \hat{\epsilon}_{4}\right), S_{4}^{1,1,2}=\frac{2}{3} d \psi^{V_{2,1,1}\left(\hat{\epsilon}_{3}, \hat{\epsilon}_{4}\right),} \\
S_{5}^{1,2,2}=-\frac{2}{3} d \psi^{V_{1,2,1}}\left(\hat{\epsilon}_{5}, \hat{\epsilon}_{6}\right), \quad S_{6}^{1,2,2}=\frac{2}{3} d \psi^{V_{1,2,1}}\left(\hat{\epsilon}_{5}, \hat{\epsilon}_{6}\right),
\end{gathered}
$$


Again, a tedious but straightforward check shows that such functions define a $\psi^{\prime}$ so that all equations (6.10) and Lemma 6.2 (3) are satisfied. Hence we may replace $\psi$ by $\psi^{\prime}$ and assume that now even (6.46) and (6.47) are satisfied. Moreover, any other good Cartan connection with the same properties is determined by functions $S_{j}^{A, k}$ which are all trivial except for the functions $S_{j}^{1,2,2}$ and $S_{k}^{1,1,2}$, with $j=1,3,4$ and $k=2,5,6$, and the functions $S_{1}^{1,1,2}$ and $S_{2}^{1,2,2}$.

These functions are uniquely determined if we require that (6.51), (6.52) and (6.53) are true. In fact, using the vanishing of all other functions and by the same arguments of before, we see that this occurs if and only if

$$
\begin{aligned}
& S_{1}^{1,1,2}=d \psi^{V_{1,1,2}}\left(\hat{\epsilon}_{3}, \hat{\epsilon}_{4}\right), \quad S_{2}^{1,2,2}=d \psi^{V_{1,2,2}}\left(\hat{\epsilon}_{5}, \hat{\epsilon}_{6}\right) \\
& S_{j}^{1,2,2}=-\frac{1}{4}\left(2 d \psi^{V_{2,2,0}}\left(\hat{\epsilon}_{j}, \hat{\epsilon}_{2}\right)+d \psi^{V_{2,2,1}}\left(\hat{\epsilon}_{j}, \hat{\epsilon}_{5}\right)+d \psi^{V_{1,2,1}}\left(\hat{\epsilon}_{j}, \hat{\epsilon}_{6}\right)\right), \\
& S_{k}^{1,1,2}=-\frac{1}{4}\left(2 d \psi^{V_{2,1,0}}\left(\hat{\epsilon}_{k}, \hat{\epsilon}_{1}\right)+d \psi^{V_{2,1,1}}\left(\hat{\epsilon}_{k}, \hat{\epsilon}_{3}\right)+d \psi^{V_{1,1,1}}\left(\hat{\epsilon}_{k}, \hat{\epsilon}_{4}\right)\right),
\end{aligned}
$$

for any $j=1,3,4$ and $k=2,5,6$.

Again, it can be checked that (6.10) and Lemma 6.2 (3) are satisfied and hence that such a $\mathfrak{g}_{\mathcal{Q}}$-valued form is a Cartan connection. The uniqueness of such connection follows immediately from (6.63), (6.64) and (6.65).

The previous lemma leads to the main result of this subsection.

Proposition 6.8. There exists a unique (globally defined) good Cartan connection $\psi_{C M}$ on $P_{\mathrm{CM}}(M)$ that satisfies (6.16) - (6.22), (6.25), (6.36) - (6.38), (6.46) (6.53). Moreover, the pair $\left(P_{\mathrm{CM}}(M), \psi_{C M}\right)$ is, generically, not isomorphic with the pair $\left(P(M), \omega_{M}\right)$ of Theorem 1.1.

Proof. By the remarks after (6.10), the existence of a unique canonical Cartan connection which satisfies the hypothesis on any trivializable open set implies the existence of a globally defined Cartan connection. To prove the second claim, let us consider the conditions which correspond to (6.11). For this, we need to determine the basis for $\mathfrak{g}_{\mathcal{Q}}^{1}+\mathfrak{g}_{\mathcal{Q}}^{2}$ which is $\mathcal{B}$-dual to the basis $\epsilon_{J}, 1 \leq J \leq 6$, of $\mathfrak{g}_{\mathcal{Q}}^{-1}+\mathfrak{g}_{\mathcal{Q}}^{-2}$.

By classical properties of graded semisimple Lie algebras (see e.g. [15], Lemma $3.15)$, the $\mathcal{B}$-dual element of an element in $\mathfrak{g}_{\mathcal{Q}}^{i}$ has to be in $\mathfrak{g}_{\mathcal{Q}}^{-i}$. In particular, the $\mathcal{B}$-dual element of $\epsilon_{1}$ is a multiple of $V_{1,1,2}$. On the other hand, the element $V_{2,1,0}$ is a grading element for $\mathfrak{g}_{\mathcal{Q}}$, i.e. for any element $X \in \mathfrak{g}_{\mathcal{Q}}^{i}$ in the same simple subalgebra of $V_{2,1,0}$, we have $\left[V_{2,1,0}, X\right]=i X$. Using this fact, the $\mathcal{B}$-norm of $V_{2,1,0}$ is immediately computed, i.e. $\mathcal{B}\left(V_{2,1,0}, V_{2,1,0}\right)=12$. So we also have

$$
\begin{gathered}
\mathcal{B}\left(\epsilon_{1}, V_{1,1,2}\right)=\frac{1}{2} \mathcal{B}\left(\epsilon_{1},\left[V_{2,1,0}, V_{1,1,2}\right]\right)=-\frac{1}{2} \mathcal{B}\left(\left[\epsilon_{1}, V_{1,1,2}\right], V_{2,1,0}\right)= \\
=-\frac{1}{2} \mathcal{B}\left(V_{2,1,0}, V_{2,1,0}\right)=-6 .
\end{gathered}
$$

This shows that the element that is $\mathcal{B}$-dual to $\epsilon_{1}$ is $-\frac{1}{6} V_{1,1,2}$. By a similar line of arguments, one can determine all the other elements $\epsilon^{A}$ of the basis which is $\mathcal{B}$-dual to $\epsilon_{J}, 1 \leq J \leq 6$. Furthermore, since (6.11) is satisfied also if we rescale all vectors $\epsilon^{A}$ by the factor $-1 / 6$, we may assume that the vectors $\left(\epsilon_{A}\right)$ are $\epsilon^{1}=V_{1,1,2}$, $\epsilon^{2}=V_{1,2,2}, \epsilon^{3}=-V_{1,1,1}, \epsilon^{4}=V_{2,1,1}, \epsilon^{5}=-V_{1,2,1}$ and $\epsilon^{6}=V_{2,2,1}$. 
At this point, it is just a matter of computing and showing that there is one condition which is, generically, not satisfied. In fact, let us set $\hat{\epsilon}_{B}=\hat{\epsilon}_{3}$ and let us evaluate the component along the vector $V_{1,1,2}$ of (6.11): using (6.13), such component becomes into

$$
\begin{gathered}
0=V_{1,1,2}^{*}\left(d \psi^{V_{1,1,2}}\left(\hat{\epsilon}_{1}, \hat{\epsilon}_{3}\right)\right)+V_{2,1,1}^{*}\left(d \psi^{V_{1,1,2}}\left(\hat{\epsilon}_{4}, \hat{\epsilon}_{3}\right)\right)= \\
-2 d \psi^{V_{1,1,2}}\left(\hat{\epsilon}_{1}, \hat{\epsilon}_{3}\right)-d \psi^{V_{1,1,2}}\left(\hat{\epsilon}_{1}, \hat{\epsilon}_{3}\right) .
\end{gathered}
$$

By (6.46), we see that our connection satisfies $(6.11)$ only if $d \psi^{V_{1,1,2}}\left(\hat{\epsilon}_{1}, \hat{\epsilon}_{3}\right)=0$. But this equality is not consequences of the conditions which characterize $\psi_{C M}$, nor it is a consequence of the linear relations implied by the identity $d^{2} \psi_{C M}=0$, as one can check with the help of a computer program like e.g. Mathematica. With the help of Frobenious theorem, one may infer that hyperbolic manifolds, for which $d \psi^{V_{1,1,2}}\left(\hat{\epsilon}_{1}, \hat{\epsilon}_{3}\right) \neq 0$, exist and are generic.

\subsection{Construction of a canonical Cartan connection on an elliptic manifold.}

Assume that $M$ is elliptic. In this case $\mathfrak{g}_{\mathcal{Q}}=\mathfrak{s l}_{3}(\mathbb{C})$ and the special basis $\left(\epsilon_{i}, V_{A, k}\right)$ is given in Appendix.

Consider a good Cartan connection $\psi$ on a trivializable open subset $\pi^{-1}(\mathcal{U}) \subset$ $P_{\mathrm{CM}}(M)$. Since $\mathfrak{g}_{\mathcal{Q}}$ is a complex Lie algebra, $\psi$ induces on $\pi^{-1}(\mathcal{U}) \subset P_{\mathrm{CM}}(M)$, the (in general, non-integrable) complex structure, determined by the endomorphisms of tangent spaces defined by

$$
J_{o}: T_{u} P_{\mathrm{CM}}(M) \rightarrow T_{u} P_{\mathrm{CM}}(M) \quad \psi\left(J_{o}(X)\right)=i \psi(X) .
$$

Notice that, by construction, $J_{o}^{*} \psi=i \psi$ and that we have the following relations between the components of $\psi$ :

$$
\begin{gathered}
J_{o}^{*} \psi^{\epsilon_{1}}=-\psi^{\epsilon_{2}}, \quad J_{o}^{*} \psi^{\epsilon_{3}}=\psi^{\epsilon_{4}}, \quad J_{o}^{*} \psi^{\epsilon_{5}}=-\psi^{\epsilon_{6}}, \\
J_{o}^{*} \psi^{V_{4,0}}=\psi^{V_{1,0}}, \quad J_{o}^{*} \psi^{V_{2,0}}=-\psi^{V_{3,0}}, \\
J_{o}^{*} \psi^{V_{1,1}}=-\psi^{V_{2,1}}, \quad J_{o}^{*} \psi^{V_{3,1}}=-\psi^{V_{4,1}}, J_{o}^{*} \psi^{V_{1,2}}=-\psi^{V_{2,2}} .
\end{gathered}
$$

So, the $\mathbb{C}$-valued forms on $\pi^{-1}(\mathcal{U}) \subset P_{\mathrm{CM}}(M)$ defined by

$$
\begin{gathered}
\varpi^{0}=\psi^{\epsilon_{1}}+i \psi^{\epsilon^{2}}, \quad \varpi^{1}=\psi^{\epsilon_{3}}-i \psi^{\epsilon^{4}}, \quad \varpi^{2}=\psi^{\epsilon_{5}}+i \psi^{\epsilon^{6}} \\
\Psi^{V_{I, 0}}=\psi^{V_{4,0}}-i \psi^{V_{1,0}}, \quad \Psi^{V_{I I, 0}}=\psi^{V_{2,0}}+i \psi^{V_{3,0}}, \quad \Psi^{V_{I, 1}}=\psi^{V_{1,1}}+i \psi^{V_{2,1}} \\
\Psi^{V_{I I, 1}}=\psi^{V_{3,1}}+i \psi^{V_{4,1}}, \quad \Psi^{V_{2}}=\psi^{V_{1,2}}+i \psi^{V_{2,2}}
\end{gathered}
$$

determine at any tangent space $T_{u} P_{\mathrm{CM}}(M)$ a basis of complex 1-forms which are holomorphic w.r.t. the complex structure $J_{o}: T_{u} P_{\mathrm{CM}}(M) \rightarrow T_{u} P_{\mathrm{CM}}(M)$. We may also consider at any point the corresponding dual holomorphic basis (i.e. a basis of elements in $T^{1,0} P_{\mathrm{CM}}(M) \subset T^{\mathbb{C}} P_{\mathrm{CM}}(M)$ ). These dual bases are given at any point by the following complex vector fields of $T^{\mathbb{C}} P_{\mathrm{CM}}(M)$ :

$$
\hat{e}_{0}=\frac{1}{2}\left(\hat{\epsilon}_{1}-i \hat{\epsilon}_{2}\right), \quad \hat{e}_{1}=\frac{1}{2}\left(\hat{\epsilon}_{3}+i \hat{\epsilon}_{4}\right), \quad \hat{e}_{2}=\frac{1}{2}\left(\hat{\epsilon}_{5}-i \hat{\epsilon}_{6}\right),
$$




$$
\begin{gathered}
\hat{V}_{I, 0}=\frac{1}{2}\left(V_{4,0}^{*}+i V_{1,0}^{*}\right), \quad \hat{V}_{I I, 0}=\frac{1}{2}\left(V_{2,0}^{*}-i V_{3,0}^{*}\right), \\
\hat{V}_{I, 1}=\frac{1}{2}\left(V_{1,1}^{*}-i V_{2,1}^{*}\right), \quad V_{I I, 1}=\frac{1}{2}\left(V_{3,1}-i V_{4,1}\right), \quad V_{2}=\frac{1}{2}\left(V_{1,2}-i V_{2,2}\right) .
\end{gathered}
$$

It is clear that any real 1 -form on $\pi^{-1}(\mathcal{U}) \subset P_{\mathrm{CM}}(M)$ can be expressed in terms of the real and imaginary parts of the $\mathbb{C}$-valued 1 -forms $\varpi^{i}$ and $\Psi^{V_{A}}$. In particular, we may express any good Cartan connection $\psi^{\prime}$ on $\pi^{-1}(\mathcal{U}) \subset P_{\mathrm{CM}}(M)$ using the $\varpi^{i}$ s and $\Psi^{V_{A}}$ 's and some suitable complex valued functions $S_{i}^{A}$. Since those expressions will turn out to be very helpful for our next computations, we write them down, for reader's convenience.

Assume that $\psi^{\prime}$ is a $\mathfrak{g}_{\mathcal{Q}}$-valued 1-form, which satisfies (6.5) and i) and ii) of Definition 6.1. Then, again, we may consider the (non-integrable) complex structure $J_{o}^{\prime}$ defined by $i \psi^{\prime}(X)=\psi\left(J_{o}^{\prime} X\right)$ and the $\mathbb{C}$-valued 1-forms $\left\{\varpi^{\prime i}, \Psi^{\prime V_{A}}\right\}$, which are defined as in (6.66)-(6.68) and are $J_{o}^{\prime}$-holomorphic at any tangent space. We may also consider the associated vector fields $\left\{\hat{e}_{i}^{\prime}, \hat{V}_{A}^{\prime}\right\}$, which are defined as in (6.69) and (6.70). These vector fields and 1-forms are written in terms of the previous one as follows

$$
\begin{gathered}
\hat{e}_{i}^{\prime}=\hat{e}_{i}+\sum_{A, \ell} S_{i}^{A} \hat{V}_{A}+\sum_{A} S_{i}^{\bar{A}} \hat{V}_{A}, \quad \hat{V}_{A}^{\prime}=\hat{V}_{A}, \\
\varpi^{\prime i}=\varpi^{i}, \quad \Psi^{\prime A}=\Psi^{A}-\sum_{i} S_{i}^{A} \varpi^{i}-\sum_{i} S_{\bar{i}}^{A} \varpi^{\bar{i}} \quad\left(\varpi^{\bar{i}} \stackrel{\text { def }}{=} \varpi^{i}, S_{\bar{i}}^{A} \stackrel{\text { def }}{=} \overline{S_{i}^{\bar{A}}}\right)
\end{gathered}
$$

for some suitable $\mathbb{C}$-valued functions $S_{i}^{A}$ and $S_{\bar{i}}^{A}$ (which are linear combinations of the original $\mathbb{R}$-valued functions $S_{i}^{A, \ell}$ ). By the same arguments of before, $\psi^{\prime}$ is a Cartan connection if and only if for any vector $V_{A}$ amongst $V_{I, 0} \stackrel{\text { def }}{=} V_{4,0}$, $V_{I I, 0} \stackrel{\text { def }}{=} V_{2,0}, V_{I, 1} \stackrel{\text { def }}{=} V_{1,1}, V_{I I, 1} \stackrel{\text { def }}{=} V_{2,1}$ and $V_{2} \stackrel{\text { def }}{=} V_{1,2}$ and any vector $e_{0} \stackrel{\text { def }}{=} \epsilon_{1}$, $e_{1} \stackrel{\text { def }}{=} \epsilon_{3}$ and $e_{2} \stackrel{\text { def }}{=} \epsilon_{5}$ we have that

$$
\left[V_{A}, e_{i}\right]=\psi^{\prime}\left(\left[\hat{V}_{A}, \hat{e}_{i}\right]\right), \quad 0=\psi^{\prime}\left(\left[\hat{V}_{A}, \overline{\hat{e}_{i}}\right]\right), \quad 0=\psi^{\prime}\left(\left[\hat{\hat{V}}_{A}, \hat{e}_{i}\right]\right)
$$

By the same arguments used to prove (6.10), it follows that $\psi^{\prime}$ is a Cartan connection if and only if

$$
\begin{gathered}
\hat{V}_{B}\left(S_{i}^{A}\right)=\sum_{j} S_{j}^{A} \varpi^{j}\left(\left[\hat{V}_{B}, \hat{e}_{i}\right]\right)-\sum_{C} S_{i}^{C} \Psi^{A}\left(\left[\hat{V}_{B}, \hat{V}_{C}\right]\right), \quad \overline{\hat{V}_{B}}\left(S_{i}^{A}\right)=0 \\
\hat{V}_{B}\left(S_{\bar{i}}^{A}\right)=-\sum_{C} S_{\bar{i}}^{C} \Psi^{A}\left(\left[\hat{V}_{B}, \hat{V}_{C}\right]\right), \quad \overline{\hat{V}_{B}}\left(S_{\bar{i}}^{A}\right)=\sum_{j} S_{\bar{j}}^{A} \overline{\varpi^{j}\left(\left[\hat{V}_{B}, \hat{e}_{i}\right]\right)}
\end{gathered}
$$

Finally, before going on with the next lemmata and construct the desired canonical Cartan connection, we want to remark that for any $\mathfrak{g}_{\mathcal{Q}}$-valued 1-form $\psi$, which satisfies i) and ii) of Definition 6.1 together with (6.5), it is possible to express $d \varpi^{0}=d\left(\omega^{1}+i \omega^{2}\right)=d\left(\psi^{\epsilon_{1}}+i \psi^{\epsilon_{2}}\right)$ in terms of the $\mathbb{C}$-valued 1-forms as follows:

$$
d \varpi^{0}=\varpi^{1} \wedge \varpi^{2}+\sigma \bar{\varpi}^{1} \wedge \bar{\varpi}^{0}+\tau \bar{\varpi}^{2} \wedge \bar{\varpi}^{0}+2 \Psi^{V_{I, 0}} \wedge \varpi^{0}
$$

+ linear combinations of $\left\{\varpi^{0} \wedge \bar{\varpi}^{0}, \varpi^{0} \wedge \varpi^{j}, \varpi^{0} \wedge \bar{\varpi}^{j}, 1 \leq j \leq 2\right\}$ 
for some suitable $\mathbb{C}$-valued functions $\sigma$ and $\tau$. Moreover, from the vanishing of

$$
d^{2} \varpi^{0}\left(\hat{e}_{0}, \overline{\hat{e}_{1}}, \overline{\hat{e}_{2}}\right)=d^{2} \varpi^{0}\left(\hat{e}_{1}, \overline{\hat{e}_{1}}, \overline{\hat{e}_{2}}\right)=d^{2} \varpi^{0}\left(\hat{e}_{2}, \overline{\hat{e}_{1}}, \overline{\hat{e}_{2}}\right)=0
$$

and using (6.3), one can check that for any good Cartan connection $\psi$ the following identities hold:

$$
d \varpi^{0}\left(\hat{e}_{0}, \overline{\hat{e}_{0}}\right)=d \varpi^{1}\left(\overline{\hat{e}_{1}}, \overline{\hat{e}_{2}}\right)=d \varpi^{2}\left(\overline{\hat{e}_{1}}, \overline{\hat{e}_{2}}\right)=0
$$

Notice that such identities could be also proved using the integrability of any elliptic CR structure.

Lemma 6.9. On any trivializable open subset $\pi^{-1}(\mathcal{U}) \subset P_{\mathrm{CM}}(M)$, there exists a good Cartan connection $\psi$, which satisfies the following conditions for any $j=0,1,2$ together with those obtained by complex conjugation:

$$
\begin{gathered}
d \varpi^{0}\left(\hat{e}_{0}, \hat{e}_{1}\right)=d \varpi^{0}\left(\hat{e}_{0}, \hat{e}_{2}\right)=d \varpi^{1}\left(\hat{e}_{1}, \hat{e}_{2}\right)=d \varpi^{2}\left(\hat{e}_{1}, \hat{e}_{2}\right)=0, \\
d \varpi^{1}\left(\frac{\hat{e}_{j}}{\hat{e}_{2}}\right)-d \varpi^{2}\left(\overline{\hat{e}_{j}}, \hat{e}_{1}\right)=d \varpi^{0}\left(\overline{\hat{e}_{j}}, \hat{e}_{0}\right)+d \varpi^{1}\left(\overline{\hat{e}_{j}}, \hat{e}_{1}\right)+d \varpi^{2}\left(\overline{\hat{e}_{j}}, \hat{e}_{2}\right)=0, \\
d \varpi^{1}\left(\hat{e}_{1}, \hat{e}_{0}\right)=d \varpi^{2}\left(\hat{e}_{2}, \hat{e}_{0}\right)=d \varpi^{1}\left(\hat{e}_{2}, \hat{e}_{0}\right)=d \varpi^{2}\left(\hat{e}_{1}, \hat{e}_{0}\right)=0,
\end{gathered}
$$

Moreover, if $\psi^{\prime}$ is another Cartan connection with the same properties and expressed in terms of $\psi$ as in (6.72), then the corresponding functions $S_{j}^{I, 0}, S_{j}^{I I, 0}$, with $j=$ 1,2 , and the functions $S_{\bar{J}}^{I, 0}, S_{\bar{J}}^{I I, 0}$, with $J=0,1,2$, are identically vanishing, while the remaining functions satisfy the relations

$$
S_{0}^{I, 0}=S_{1}^{I I, 1}=S_{2}^{I, 1}, \quad S_{1}^{I I, 0}=-S_{1}^{I, 1}=S_{2}^{I I, 1} .
$$

Finally, for any good Cartan connection $\psi$, which satisfies (6.75) -(6.77) the following identities hold for any $J=0,1,2$ :

$$
d \varpi^{0}\left(\hat{e}_{0}, \overline{\hat{e}_{J}}\right)=d \Psi^{I, 0}\left(\hat{e}_{1}, \hat{e}_{2}\right)=d \Psi^{I, 0}\left(\overline{\hat{e}_{1}}, \overline{\hat{e}_{2}}\right) .
$$

Proof. As in the proof of Lemma 6.5, we fix a good Cartan connection $\psi$ and we consider a new $\mathfrak{g}_{\mathcal{Q}}$-valued 1-form $\psi^{\prime}$ defined by some functions $S_{J}^{A, k}$ as in (6.72). We have to show that it is possible to choose the functions $S_{J}^{A, k}$ so that (6.75) and (6.76) are satisfied and so that $\psi^{\prime}$ is a Cartan connection. By the same arguments as in Lemma 6.5, it turns out this occurs if and only if for $i=1,2$

$$
\begin{gathered}
S_{i}^{I, 0}=\frac{1}{2} d \varpi^{0}\left(\hat{e}_{0}, \hat{e}_{i}\right), \\
S_{1}^{I I, 0}=\frac{1}{2} d \varpi^{0}\left(\hat{e}_{0}, \hat{e}_{2}\right)-d \varpi^{1}\left(\hat{e}_{1}, \hat{e}_{2}\right), \\
S_{2}^{I I, 0}=-\frac{1}{2} d \varpi^{0}\left(\hat{e}_{0}, \hat{e}_{1}\right)-d \varpi^{2}\left(\hat{e}_{1}, \hat{e}_{2}\right), \\
S_{\bar{j}}^{I, 0}=-\frac{1}{4}\left(d \varpi^{0}\left(\overline{\hat{e}_{j}}, \hat{e}_{0}\right)+d \varpi^{1}\left(\overline{\hat{e}_{j}}, \hat{e}_{1}\right)+d \varpi^{2}\left(\overline{\hat{e}_{j}}, \hat{e}_{2}\right)\right),
\end{gathered}
$$




$$
\begin{gathered}
S_{\bar{j}}^{I I, 0}=\frac{1}{2}\left(d \varpi^{2}\left(\bar{e}_{j}, \hat{e}_{1}\right)-d \varpi^{1}\left(\overline{\hat{e}_{j}}, \hat{e}_{2}\right)\right) \\
S_{0}^{I, 0}-S_{1}^{I I, 1}=d \varpi^{1}\left(\hat{e}_{1}, \hat{e}_{0}\right), S_{0}^{I I, 0}-S_{2}^{I, 1}=d \varpi^{1}\left(\hat{e}_{2}, \hat{e}_{0}\right), \\
S_{0}^{I I, 0}+S_{1}^{I, 1}=d \varpi^{2}\left(\hat{e}_{0}, \hat{e}_{1}\right), S_{0}^{I, 0}-S_{2}^{I I, 1}=d \varpi^{2}\left(\hat{e}_{2}, \hat{e}_{0}\right) .
\end{gathered}
$$

The system given by the equations (6.80) - (6.86) has maximal rank and it is therefore solvable at any point. Moreover, with some straightforward computations based on (6.10') and (6.12), it can be checked that any set of functions which satisfy that system at the points of a submanifold transversal to all fibers can be smoothly extended to functions which solve all equations (6.80) - (6.86) at all points and that, at the same time, they satisfy all equations (6.10') and condition (3) of Lemma 6.2. From this we get the existence of a Cartan connection which satisfies all requirements. The second claim follows immediately from (6.80) - (6.86). The last claim can be checked by evaluating $d^{2} \varpi^{0}\left(\overline{\hat{e}_{J}}, \hat{e}_{1}, \hat{e}_{2}\right)$, with $J=0,1,2$, $d^{2} \varpi^{0}\left(\hat{e}_{0}, \hat{e}_{1}, \hat{e}_{2}\right)$ and $d^{2} \varpi^{0}\left(\hat{e}_{0}, \overline{\hat{e}_{1}}, \overline{\hat{e}_{2}}\right)$ and recalling that they have to be all identically vanishing by the triviality of the operator $d^{2}$.

Lemma 6.10. On any trivializable open subset $\pi^{-1}(\mathcal{U}) \subset P_{\mathrm{CM}}(M)$, there exists good Cartan connection $\psi$, which satisfies (6.75) - (6.77) and the following conditions together with those obtained by complex conjugation:

$$
\begin{gathered}
d \Psi^{V_{I I, 0}}\left(\hat{e}_{1}, \hat{e}_{2}\right)=0 . \\
d \Psi^{V_{I I, 0}}\left(\hat{e}_{1}, \hat{e}_{0}\right)+d \Psi^{V_{I, 0}}\left(\hat{e}_{2}, \hat{e}_{0}\right)=d \Psi^{V_{I, 0}}\left(\hat{e}_{1}, \hat{e}_{0}\right)-d \Psi^{V_{I I, 0}}\left(\hat{e}_{2}, \hat{e}_{0}\right)=0 .
\end{gathered}
$$

Moreover, if $\psi^{\prime}$ is another good Cartan connections with the same properties and obtained from $\psi$ as in (6.72), then all corresponding functions $S_{J}^{A, k}$ are vanishing except for the functions which appear in (6.78) $)_{1}$ and the functions $S_{0}^{A, 1}, S_{\bar{j}}^{A, 1}$ and $S_{J}^{I, 2}$, with $A=I, I I, j=1,2$ and $J=0,1,2$, among which the following relations have to be satisfied:

$$
S_{0}^{I, 1}=-\frac{1}{2} S_{1}^{I, 2}, \quad S_{0}^{I I, 1}=\frac{1}{2} S_{2}^{I, 2}
$$

Proof. Following the same line of arguments of the previous lemma and of Lemma 6.6 and using (6.78), one may check that (6.87) and (6.88) are satisfied if and only if

$$
\begin{gathered}
S_{0}^{I I, 0}=S_{2}^{I I, 1}=-S_{1}^{I, 1}=\frac{1}{4} d \Psi^{V_{I I, 0}}\left(\hat{e}_{1}, \hat{e}_{2}\right) \\
2 S_{0}^{I I, 1}-S_{2}^{I, 2}=d \Psi^{V_{I I, 0}}\left(\hat{e}_{1}, \hat{e}_{0}\right)+d \Psi^{V_{I, 0}}\left(\hat{e}_{2}, \hat{e}_{0}\right) \\
2 S_{0}^{I, 1}+S_{1}^{I, 2}=d \Psi^{V_{I, 0}}\left(\hat{e}_{1}, \hat{e}_{0}\right)-d \Psi^{V_{I I, 0}}\left(\hat{e}_{2}, \hat{e}_{0}\right) .
\end{gathered}
$$

As in the previous proof, we notice that (6.90) - (6.92) is a maximal rank system and that, using $\left(6.10^{\prime}\right)$, it is possible to determine smooth solutions which solve the system at any point and which, at the same time, satisfy all (6.10') and Lemma 6.2 (3). This gives the existence of a good Cartan connection which satisfies all requirements. The second claim follows immediately from (6.90) - (6.92). 
Lemma 6.11. On any trivializable open subset $\pi^{-1}(\mathcal{U}) \subset P_{\mathrm{CM}}(M)$, there exists a unique Cartan connection $\psi$, which satisfies (6.75) - (6.77), (6.87), (6.88) and the next conditions for $J=0,1,2$, together with all equations that can be obtained by complex conjugation:

$$
\begin{aligned}
& d \Psi^{V_{I, 1}}\left(\hat{e}_{1}, \hat{e}_{2}\right)=d \Psi^{V_{I I, 1}}\left(\hat{e}_{1}, \hat{e}_{2}\right)=0, \\
& d \varpi^{2}\left(\overline{\hat{e}_{J}}, \hat{e}_{0}\right)+d \Psi^{V_{I I, 0}}\left(\overline{\hat{e}_{J}}, \hat{e}_{2}\right)-d \Psi^{V_{I, 0}}\left(\overline{\hat{e}_{J}}, \hat{e}_{1}\right)=0, \\
& d \varpi^{1}\left(\overline{\hat{e}_{J}}, \hat{e}_{0}\right)+d \Psi^{V_{I I, 0}}\left(\overline{\hat{e}_{J}}, \hat{e}_{1}\right)+d \Psi^{V_{I, 0}}\left(\overline{\hat{e}_{J}}, \hat{e}_{2}\right)=0, \\
& d \Psi^{V_{I, 2}}\left(\hat{e}_{1}, \hat{e}_{2}\right)=2 d \Psi^{V_{I, 0}}\left(\overline{\hat{e}_{J}}, \hat{e}_{0}\right)+d \Psi^{V_{I I, 1}}\left(\overline{\hat{e}_{J}}, \hat{e}_{1}\right)+d \Psi^{V_{I, 1}}\left(\overline{\hat{e}_{J}}, \hat{e}_{2}\right)=0 .
\end{aligned}
$$

Proof. Following the same line of arguments of the Lemma 6.6, one can see that any good Cartan connection for which (6.75) - (6.77),(6.87) and (6.88) hold, satisfies also (6.93) if and only if

$$
\begin{aligned}
& S_{1}^{I, 2}=-\frac{2}{3} d \Psi^{V_{I, 1}}\left(\hat{e}_{1}, \hat{e}_{2}\right)+\frac{2}{3} \sum_{j=1,2} S_{\bar{j}}^{I, 1} \overline{d \varpi^{j}}\left(\hat{e}_{1}, \hat{e}_{2}\right), \\
& S_{2}^{I, 2}=\frac{2}{3} d \Psi^{V_{I, 1}}\left(\hat{e}_{1}, \hat{e}_{2}\right)+\frac{2}{3} \sum_{j=1,2} S_{\bar{j}}^{I I, 1} d \overline{\varpi^{j}}\left(\hat{e}_{1}, \hat{e}_{2}\right),
\end{aligned}
$$

On the other, if we assume that $\psi^{\prime}$ is also a Cartan connection (and hence the functions $S_{J}^{A, k}$ satisfy $\left.\left(6.10^{\prime}\right)\right)$, then, by $(6.10),(6.12),(6.13)$ and (6.78), we must have also that

$$
\begin{gathered}
V_{I, 1}^{*}\left(S_{\bar{j}}^{I, 1}\right)=S_{\bar{j}}^{I, 0}=0 \\
S_{0}^{I, 0}=S_{2}^{I, 1}=S_{1}^{I I, 1}=-V_{I, 1}^{*}\left(S_{1}^{I, 2}\right) \\
=-V_{I, 1}^{*}\left(-\frac{2}{3} d \Psi^{V_{I, 1}}\left(\hat{e}_{1}, \hat{e}_{2}\right)+\frac{2}{3} \sum_{j=1,2} S_{\bar{j}}^{I, 1} d \overline{\varpi^{j}}\left(\hat{e}_{1}, \hat{e}_{2}\right)\right)=0 .
\end{gathered}
$$

Using (6.99), we get that $\psi^{\prime}$ satisfies also (6.94) - (6.96) if and only if

$$
\begin{gathered}
S_{\bar{J}}^{I, 1}=-\frac{1}{3}\left(d \varpi^{2}\left(\overline{\hat{e}_{J}}, \hat{e}_{0}\right)+d \Psi^{V_{I I, 0}}\left(\overline{\hat{e}_{J}}, \hat{e}_{2}\right)-d \Psi^{V_{I, 0}}\left(\overline{\hat{e}_{J}}, \hat{e}_{1}\right)\right) \\
S_{\bar{J}}^{I I, 1}=-\frac{1}{3}\left(d \varpi^{1}\left(\overline{\hat{e}_{J}}, \hat{e}_{0}\right)+d \Psi^{V_{I I, 0}}\left(\overline{\hat{e}_{J}}, \hat{e}_{1}\right)+d \Psi^{V_{I, 0}}\left(\overline{\hat{e}_{J}}, \hat{e}_{2}\right)\right) .
\end{gathered}
$$

With a straightforward check of (6.10') and Lemma $6.2(3)$, one can see that it is possible to construct a $\psi^{\prime}$ which satisfies (6.99) - (6.101) and hence one for which (6.94) and (6.95) are true. Then, coming back to (6.93), the previous remarks imply that the functions $S_{\bar{j}}^{I, 1}, j=1,2$, have to be vanishing and hence that a good Cartan connection, for which (6.93) is true, is determined by functions $S_{J}^{A, k}$ such that

$$
S_{1}^{I, 2}=-\frac{2}{3} d \Psi^{V_{I, 1}}\left(\hat{e}_{1}, \hat{e}_{2}\right), \quad S_{2}^{I, 2}=\frac{2}{3} d \Psi^{V_{I, 1}}\left(\hat{e}_{1}, \hat{e}_{2}\right) .
$$

Another check based on (6.10') and Lemma 6.2 (3), shows that these conditions give a Cartan connection and hence that we may assume that (6.93) - (6.95) are 
true. From (6.99) - (6.102), we also have that any other good Cartan connection with the same properties has all functions $S_{J}^{A, k}$ vanishing except for the functions $S_{\bar{J}}^{I, 2}, J=0,1,2$, and for the function $S_{0}^{I, 2}$. These functions are uniquely determined if we require that also (6.96) is satisfied. In fact this occurs if and only if

$$
\begin{gathered}
S_{0}^{I, 2}=d \Psi^{V_{I, 0}}\left(\hat{e}_{1}, \hat{e}_{2}\right) \\
S_{\bar{J}}^{I, 2}=-\frac{1}{4}\left(2 d \Psi^{V_{I, 0}}\left(\overline{\hat{e}_{J}}, \hat{e}_{0}\right)+d \Psi^{V_{I I, 1}}\left(\overline{\hat{e}_{J}}, \hat{e}_{1}\right)+d \Psi^{V_{I, 1}}\left(\overline{\hat{e}_{J}}, \hat{e}_{2}\right)\right) .
\end{gathered}
$$

Another check of the validity (6.10') and of Lemma 6.2 (3) gives the existence of such good Cartan connection and (6.103) and (6.104) give also the uniqueness.

The previous lemma provides immediately the existence of a canonical Cartan connection for any elliptic manifold. In fact,

Proposition 6.12. There exists a unique (globally defined) good Cartan connection $\psi_{C M}$ on $P_{\mathrm{CM}}(M)$ that satisfies (6.74), (6.75) - (6.77), (6.79), (6.87), (6.88), (6.94) - (6.96). Moreover, the pair $\left(P_{\mathrm{CM}}(M), \psi_{C M}\right)$ is, generically, not isomorphic with the pair $\left(P(M), \omega_{M}\right)$ of Theorem 1.1.

Proof. By the remarks after (6.10), the existence of a unique canonical Cartan connection which satisfies the hypothesis on any trivializable open set implies the existence of a globally defined Cartan connection. For the second claim, it suffices to observe that the component of (6.11) along the vector $V_{I, 2}$ with $\epsilon_{B}=\epsilon_{3}$ is generically not satisfied. Since the computations to explicitate such component and to check that the corresponding condition is not satisfied are are very similar to those given in the proof of Proposition 6.8, we omit them.

\section{APPENDix}

A special basis for $\mathfrak{g}_{\mathcal{Q}}$ (hyperbolic case)

It is known (see e.g. [16]) that in this case $\mathfrak{g}_{\mathcal{Q}}$ is a Lie algebra isomorphic to

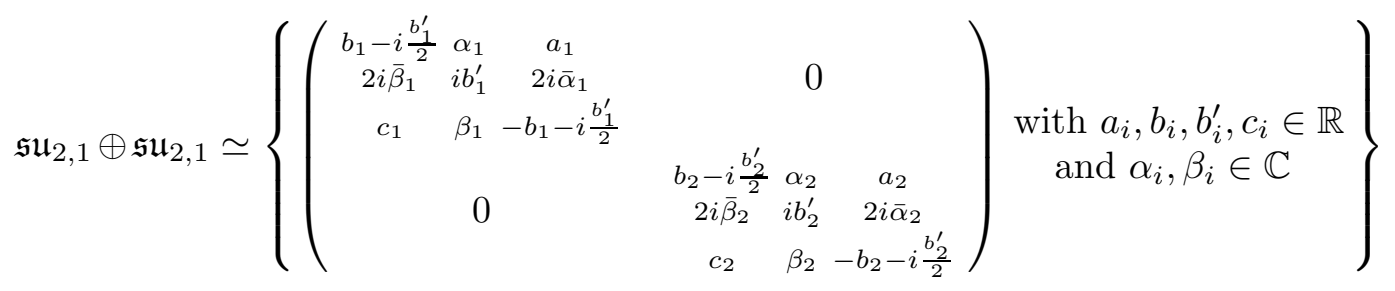

The basis we consider for such Lie algebra is the following:

$$
\begin{aligned}
& \epsilon_{1}=\left(\begin{array}{ccccc}
0 & 0 & -1 \\
0 & 0 & 0 \\
0 & 0 & 0
\end{array}\right.
\end{aligned}
$$

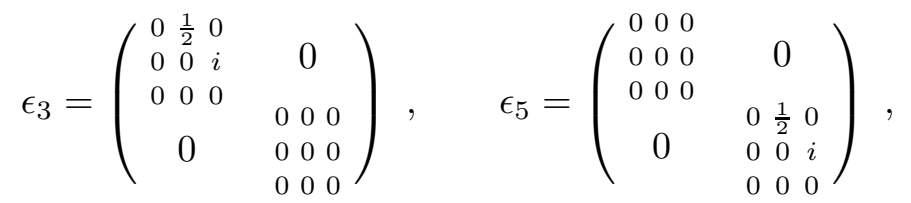




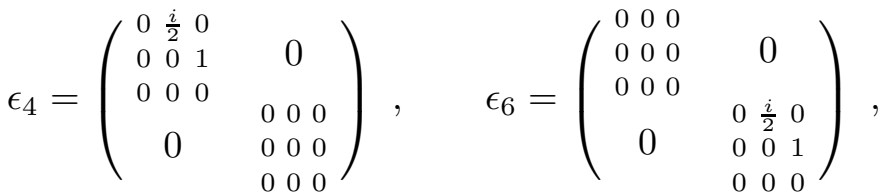

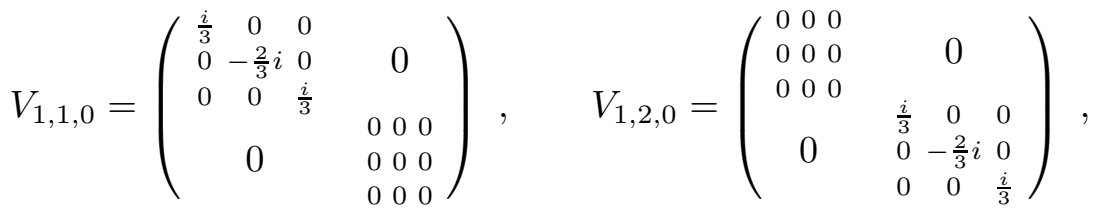

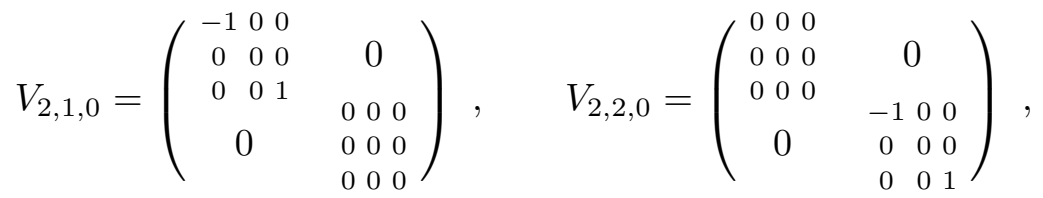

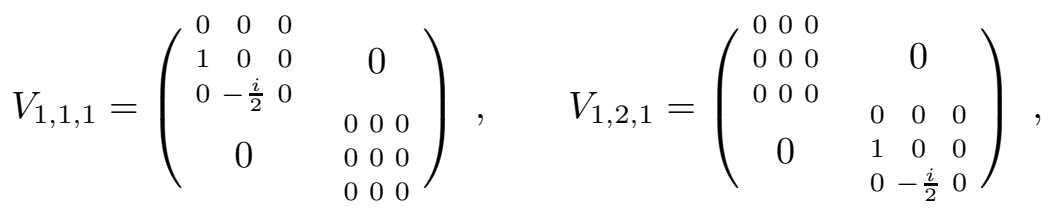

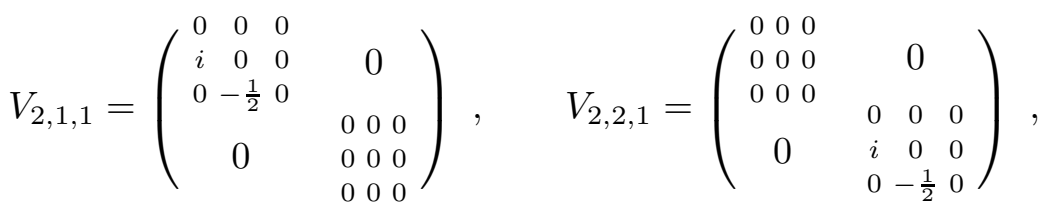

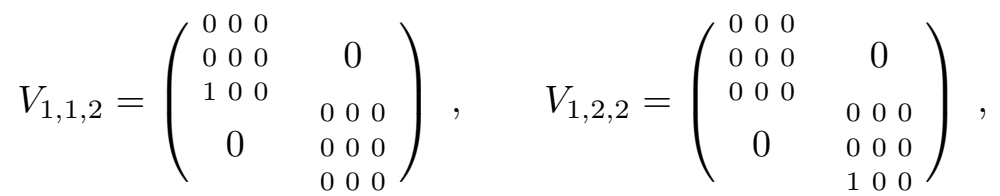

A special basis for $\mathfrak{g}_{\mathcal{Q}}$ (elliptic case)

It is known (see e.g. [16]) that in this case $\mathfrak{g}_{\mathcal{Q}}$ is a Lie algebra isomorphic to

$$
\mathfrak{s l}_{3}(\mathbb{C})=\left\{\left(\begin{array}{lll}
\alpha_{11} & \alpha_{12} & \alpha_{13} \\
\alpha_{21} & \alpha_{22} & \alpha_{23} \\
\alpha_{31} & \alpha_{32} & \alpha_{33}
\end{array}\right), \alpha_{i j} \in \mathbb{C}, \alpha_{11}+\alpha_{22}+\alpha_{33}=0\right\} \text {. }
$$

The basis we consider for such Lie algebra is the following:

$$
\begin{array}{rlrl}
\epsilon_{1} & =\left(\begin{array}{lll}
0 & 0 & 1 \\
0 & 0 & 0 \\
0 & 0 & 0
\end{array}\right), & \epsilon_{2} & =\left(\begin{array}{lll}
0 & 0 & i \\
0 & 0 & 0 \\
0 & 0 & 0
\end{array}\right), \\
\epsilon_{3} & =\left(\begin{array}{lll}
0 & \frac{1}{2} & 0 \\
0 & 0 & i \\
0 & 0 & 0
\end{array}\right), & \epsilon_{5}=\left(\begin{array}{ccc}
0 & -\frac{i}{2} & 0 \\
0 & 0 & -1 \\
0 & 0 & 0
\end{array}\right), \\
\epsilon_{4}=\left(\begin{array}{ccc}
0 & -\frac{i}{2} & 0 \\
0 & 0 & 1 \\
0 & 0 & 0
\end{array}\right), & \epsilon_{6}=\left(\begin{array}{ccc}
0 & \frac{1}{2} & 0 \\
0 & 0 & -i \\
0 & 0 & 0
\end{array}\right),
\end{array}
$$




$$
\begin{array}{ccc}
V_{1,0}= & \left(\begin{array}{ccc}
i & 0 & 0 \\
0 & 0 & 0 \\
0 & 0 & -i
\end{array}\right), V_{2,0}=\left(\begin{array}{ccc}
-\frac{i}{3} & 0 & 0 \\
0 & \frac{2 i}{3} & 0 \\
0 & 0 & -\frac{i}{3}
\end{array}\right), \\
V_{3,0} & =\left(\begin{array}{ccc}
\frac{1}{3} & 0 & 0 \\
0 & -\frac{2}{3} & 0 \\
0 & 0 & \frac{1}{3}
\end{array}\right), V_{4,0}=\left(\begin{array}{ccc}
-1 & 0 & 0 \\
0 & 0 & 0 \\
0 & 0 & 1
\end{array}\right), \\
V_{1,1}=\left(\begin{array}{ccc}
0 & 0 & 0 \\
1 & 0 & 0 \\
0 & -\frac{i}{2} & 0
\end{array}\right), & V_{3,1}=\left(\begin{array}{ccc}
0 & 0 & 0 \\
-i & 0 & 0 \\
0 & \frac{1}{2} & 0
\end{array}\right), \\
V_{2,1} & =\left(\begin{array}{ccc}
0 & 0 & 0 \\
i & 0 & 0 \\
0 & \frac{1}{2} & 0
\end{array}\right), & V_{4,1}=\left(\begin{array}{ccc}
0 & 0 & 0 \\
1 & 0 & 0 \\
0 & \frac{i}{2} & 0
\end{array}\right), \\
V_{1,2}=\left(\begin{array}{ccc}
0 & 0 & 0 \\
0 & 0 & 0 \\
-1 & 0 & 0
\end{array}\right), & V_{2,2}=\left(\begin{array}{ccc}
0 & 0 & 0 \\
0 & 0 & 0 \\
-i & 0 & 0
\end{array}\right)
\end{array}
$$

\section{REFERENCES}

[1] D. V. Alekseevsky and A. F. Spiro, Prolongations of Tanaka structures and regular CR structures, in "Selected Topics in Cauchy-Riemann Geometry" (ed S. Dragomir), Quaderni di Matematica vol. 9, Seconda Università degli Studi di Napoli - Napoli, 2001, pp. 3-37.

[2] A. Boggess, CR manifolds and the Tangential Cauchy-Riemann Complex, Studies in Advanced Mathematics, CRC Press, Boca Raton, 1991.

[3] A. Čap and M. Eastwood, Some Special Geometry in Dimension Six, preprint ArXiv: math.DG/003059 (2000).

[4] S.S. Chern, J. Moser, Real Hypersurfaces in Complex Manifolds, Acta Math. 133 (1974), 219-271.

[5] A. Čap and H. Schichl, Parabolic Geometries and Canonical Cartan Connections, Hokkaido Math. J. 29 (2000), no. 3, 453-505.

[6] A. Čap and G. Schmalz, Partially integrable almost $C R$ manifolds of $C R$ dimension and codimension two, in "Lie groups, Geometric Structures and Differential Equations - One Hundred Years after Sophus Lie" (T. Morimoto, H. Sato and K. Yamaguchi eds.), Adv. Stud. in Pure Math. vol. 37, 2002.

[7] V. V. Ezhov, A. V. Isaev and G. Schmalz, Invariants of Elliptic and Hyperbolic CRstructures of codimension 2, Intern. J. of Math. 10 (1999), no. 1, 1-52.

[8] V. V. Ezhov and G. Schmalz, Holomorphic automorphisms of quadrics, Math. Z. 216 (1994), no. 3, 453-470.

[9] V. V. Ezhov and G. Schmalz, Normal form and two-dimensional chains of an elliptic CR surface in $\mathbb{C}^{4}$, J. Geom. Anal. 6 (1996), no. 4, 495-529.

[10] V. V. Ezhov and G. Schmalz, Infinitesimale Starrheit hermitscher Quadriken in allgemeiner Lage, Math. Nachr. 204 (1999), 41-60.

[11] V. V. Ezhov and G. Schmalz, Automorphisms of nondegenerate CR quadrics and Siegel domains. Explicit description., J. Geom. Anal. 11 (2001), no. 3, 441-467.

[12] V. V. Ezhov and G. Schmalz, Linearization of isotropic automorphisms of non-quadratic elliptic $C R$ manifolds in $\mathbb{C}^{4}$, in "Geometric analysis and non-linear partial differential equations" (S. Hildebrandt and H. Karcher eds.), Springer Verlag, 2002.

[13] S. Kobayashi, K. Nomizu, Foundations of Differential Geometry, vol. I, Interscience Publ., 1963.

[14] A. V. Loboda, Generic real analytic manifolds of codimension 2 in $\mathbb{C}^{4}$ and their biholomorphic mappings, Izv. Akad. Nauk SSSR (Ser. Math.) 52, no. 5, 970-990; Engl. transl. in Math. USSR. Izv. 33 (1989), no. 2, 295-315.

[15] C. Medori, M. Nacinovich, Levi-Tanaka algebras and homogeneous CR manifolds, Compositio Mathematica 109 ((1997)), 195-250. 
[16] G. Schmalz, Remarks on CR-manifolds of Codimension 2 in $\mathbb{C}^{4}$, Proceeding Winter School Geometry and Physics, Srní,1998, Supp. Rend. Circ. Matem. Palermo, Ser. II 59 (1999), 171-180.

[17] R. W. Sharpe, Differential Geometry, Springer, 1996.

[18] G. Schmalz and J. Slovák, The Geometry of Hyperbolic and Elliptic CR manifolds of codimension two, Asian J. Math. 4 (2000), no. 3, 565-598.

[19] G. Schmalz and J. Slovák, Addendum to: The Geometry of Hyperbolic and Elliptic CR manifolds of codimension two, Asian J. Math. 7 (2003), no. 3, 303-306.

[20] N. Tanaka, On the equivalence problem associated with simple graded Lie algebras, Hokkaido Math. J. 8 (1979), 131-190.

Gerd Schmalz

Mathematisches Institut

RHEINISCHE FRIEDERICH-WILHELMS-UNIVERSITÄT

BERINGSTRASSE 1

53115 BONN

GERMANY

E-mail address: schmalz@math.uni-bonn.de
ANDREA SPIRO

Dipartimento di Matematica e Informatica

Università di CAMERINO

Via Madonna delle Carceri

I-62032 CAMERINO (MACERATA)

ITALY

E-mail address: andrea.spiro@unicam.it 